Floor Sieverink

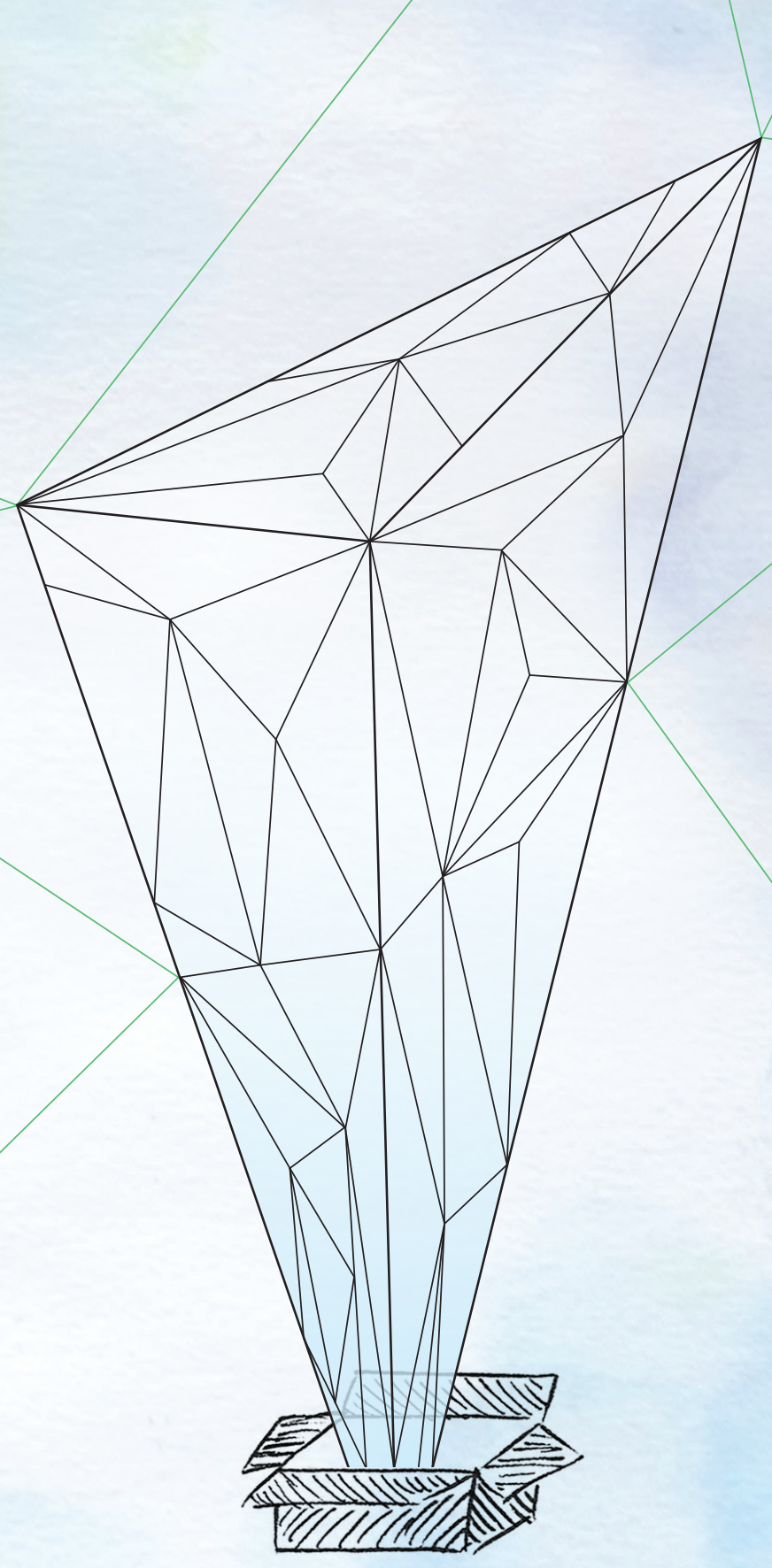

\title{
Opening the Black Box of eHealth
}

A mixed methods approach for the evaluation of personal health records 
Opening the Black Box of eHealth

A Mixed Methods Approach for the Evaluation of Personal Health Records

Floor Sieverink 
Thesis, University of Twente, 2017

(C) Floor Sieverink, Enschede, the Netherlands

ISBN: 978-90-365-4417-7

DOI: 10.3990/1.9789036544177

Cover design: Esther Scheide, www.proefschriftomslag.nl

Printed by Gildeprint, the Netherlands 


\title{
OPENING THE BLACK BOX OF EHEALTH
}

\section{A MIXED METHODS APPROACH FOR THE EVALUATION OF PERSONAL HEALTH RECORDS}

\author{
PROEFSCHRIFT
}

ter verkrijging van

de graad van doctor aan de Universiteit Twente, op gezag van de rector magnificus,

prof. dr. T.T.M. Palstra,

volgens besluit van het College voor Promoties

in het openbaar te verdedigen

op donderdag 14 december om 10.45 uur

door

Floor Sieverink

geboren op 13 april 1986

te Haaksbergen 
Dit proefschrift is goedgekeurd door de promotoren prof. dr. J.E.W.C. van Gemert-Pijnen en prof. dr. R. Sanderman en co-promotor dr. S.M. Kelders. 


\section{Samenstelling promotiecommissie}

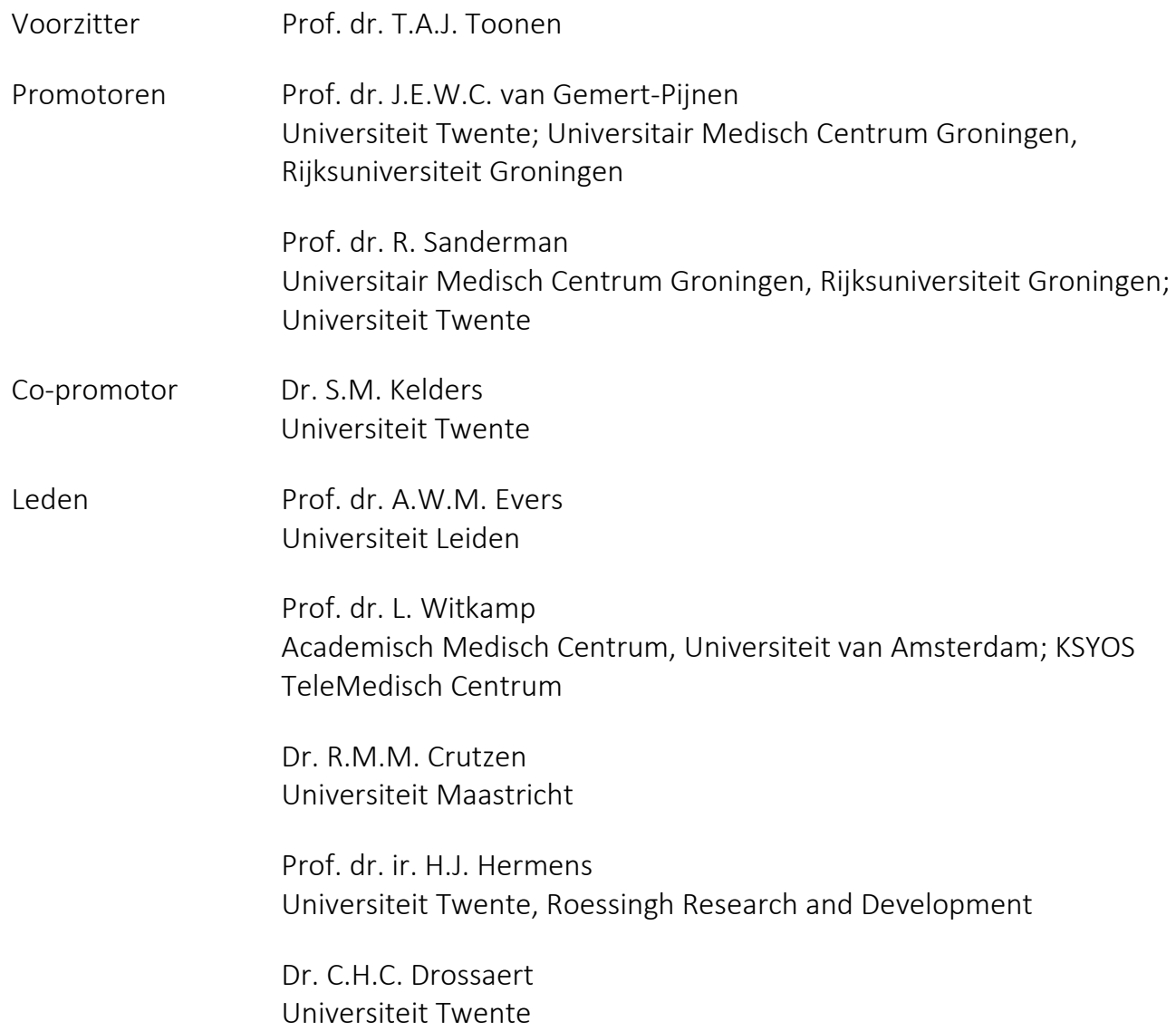





\section{Contents}

Chapter $1 \quad$ General introduction

Part 1

Chapter 2

Chapter 3

\section{Part 2}

Chapter $4 \quad$ The Added Value of Log Data Analyses of the Use of a Personal Health Record for Patients with Type 2 Diabetes Mellitus: Preliminary Results

Chapter 5 The Diffusion of a Personal Health Record for Patients with Type 2 Diabetes Mellitus in Primary Care

Chapter 6 Evaluating the implementation of a Personal Health Record for chronic primary and secondary care: A mixed methods approach

Chapter $7 \quad$ General discussion

The Personal Health Record e-Vita

Samenvatting (Summary in Dutch)

Dankwoord

Publications \& Other Output 



\section{Chapter 1}

\section{General Introduction}

Partially based on:

F. Sieverink et al. Evaluating eHealth. In J. van Gemert-Pijnen,

H. Kip, S. Kelders, R. Sanderman (Eds.)

eHealth Technology: Theory, Development, and Evaluation (in press) 


\begin{abstract}
Annette is a 45 years old diabetes nurse in a general practice in the north of the Netherlands. During the day, Annette has a busy schedule including many consultations with patients with type 2 diabetes mellitus (T2DM). She has a great variety of tasks, such as educating newly diagnosed patients and providing lifestyle- and medication advice.

Usually, Annette sees her patients every three months for a consultation. During these appointments, Annette monitors the patients' blood pressure, heart rate, weight, and the waist circumference. Furthermore, she checks the patients' feet to check for any diseaserelated problems. By performing these measurements, Annette is able to monitor the health status of the patients and to provide personalized advice.

Another task of Annette is to coach her patients in reaching their health-related goals, such as losing weight or improving their physical condition. Patients might then need support for setting achievable goals, creating a motivational action plan or providing support in dietary advice. All information is collected and stored in the electronic health record of the general practice and can be accessed by the general practitioner (GP), who is ultimately responsible for the care of the patient. The GP sees the patient once a year for an annual check-up.

Annette often finds it challenging to perform all these tasks in the brief time that she has for every patient. As a result, her workload is very high. Furthermore, Annette believes that some of the tasks and responsibilities she has, can be shared with or handed over to the patient as well. She believes that this self-management support might even improve the patients' health and wellbeing.

Based on one of the cases from the massive open online course (MOOC) eHealth: Combining Psychology, Technology and Health (https://www.futurelearn.com/courses/ehealth)
\end{abstract}

\title{
Challenges in Chronic Care
}

Due to the aging population and the growing prevalence of chronic diseases, the challenges as described above are becoming increasingly common for care professionals like Annette. A chronic disease can be defined as follows:

Diseases which have one or more of the following characteristics: they are permanent, leave residual disability, are caused by nonreversible pathological alteration, require special training of the patient for rehabilitation, or may be expected to require a long period of supervision, observation, or care [1].

In 2012, worldwide 23 million people died from having a chronic disease, or more specifically: a cardiovascular disease, diabetes or respiratory diseases, such as chronic obstructive pulmonary disease (COPD) [2]. These chronic conditions were responsible for $37 \%$ of all deaths in the Netherlands in that year [3]. In 2015, over 1.8 million people (approximately $11 \%$ of the Dutch population) were registered as having type 2 diabetes mellitus (T2DM), 
congestive heart failure (CHF) and/or COPD, and it is expected that these numbers will only grow in the upcoming years [4-6].

With this growing prevalence, a number of issues arise. For example, due to a reimbursement shift from secondary to primary care, primary care providers (e.g., general practitioners and practice nurses) are responsible for a growing number of tasks concerning the treatment and counselling of chronically ill people. At the same time, the aging population causes a decrease in the number of care providers to deal with this shift.

Furthermore, the relationship between patients and their care providers traditionally consists of a great dependence of the patient on the care provider, which can impose a burden on healthcare [7] in terms of time and costs. Also, the healthcare system largely focuses on acute illnesses, resulting in a mismatch with the needs of patients with chronic diseases for effective clinical management, psychological support, and information [8]. In this light, ageing with one or more chronic diseases is becoming normal and 'being healthy' cannot be seen anymore as "a state of complete, physical, mental, and social well-being and not merely the absence of disease or infirmity", as implied by the WHO [9]. Therefore, Huber et al. make a plea for defining health as "the ability to adapt and to self-manage" [10].

Sustainable solutions are needed to effectuate a transformation in health care delivery and to support the shifts from 1 ) institutionalized (secondary) care to (primary) home care; 2) acute episodic care to a more continuous chronic care; and 3) the patient as a passive recipient of care to an active patient who is able to self-manage [11]. In that view, chronic disease management can be seen as a set of interrelated services that spans the continuum from prevention and self-management, to intramural care for patients with chronic diseases $[8,12,13]$. Several strategies have been proposed for that purpose, including the integrated care approach for the development of personalized, structured, and multidisciplinary care plans that explain the essential steps for the care of patients with special needs [14]. These integrated care pathways have the potential to create greater efficiency and value, benefiting the care provider, the patient, as well as the greater context of the care system.

Technology-based innovations (such as eHealth) are major drivers in this transformation of care delivery. However, the actual implementation of such innovations can be very challenging. This thesis will focus on the evaluation of the implementation of an electronic personal health record (PHR) for patients with T2DM, CHF, or COPD. To understand the used evaluation approach, first it is important to elaborate on some important definitions and approaches regarding eHealth and evaluation that were used. 


\section{eHealth in Chronic Care}

For several years, eHealth technologies have played an increasingly important role in providing Internet-based disease management, e.g. by providing self-management support, in facilitating information exchange among professionals and with patients, and in monitoring the performance of disease management programs [15]. The number of definitions for eHealth that go around are innumerable, but a frequently used definition has been proposed by Eysenbach:

e-health is an emerging field in the intersection of medical informatics, public health and business, referring to health services and information delivered or enhanced through the Internet and related technologies. In a broader sense, the term characterizes not only a technical development, but also a state-of-mind, a way of thinking, an attitude, and a commitment for networked, global thinking, to improve health care locally, regionally, and worldwide by using information and communication technology [16].

This definition characterizes eHealth as much more than just 'a thing' or a tool: it is about creating and evaluating an infrastructure for knowledge dissemination, communication and the organization of care. This will not occur by just offering a technology, but it rather demands a careful embedding of the technology into the care process, with attention for its added value in a certain context. Creating sustainable eHealth technologies thus requires a holistic development and evaluation approach that takes into account the triad between the technology, its users and the context of implementation.

\section{Personal Health Records}

Personal Health Records (PHRs) or patient portals/platforms are seen as promising technologies in an integrated care approach to engage patients in their own health care and to support them in managing their personal health information that care providers can use as well in clinical decision making [17-19]. PHRs are frequently defined as:

"an application through which individuals can access, manage, and share their health information and that of others for whom they are authorized, in a private, secure and confidential environment" [20].

PHRs capture health data as collected by the patient and provide information related to the care of that patient. Therefore, they should not be confused with electronic health records (EHRs), or systems that serve the information needs of care professionals [18]. To support patients in taking a more active role in improving and maintaining their own health however, a PHR must be more than just a repository for health information, as the definition implies [18]. Therefore, potential functions of current PHRs are not limited to sharing clinical and personal data as provided by the care provider (e.g., history, test results, treatment 
information, an overview of appointments), but may also include services for selfmanagement support; patient-provider communication; peer support; monitoring health behaviour data (e.g., via health-related equipment such as weighing scales, wristbands, and smartwatches [7]); and education regarding the disease [21]. By combining services for datasharing and self-management support, PHRs have the potential to enhance chronic care delivery that demand a new way of thinking from both patients and caregivers. After all, PHRs allow for a more continuous chronic care delivery that supports the patient to become an active participant that is able to self-manage. Therefore, in this thesis, such PHRs are being considered as eHealth technologies.

Several potential benefits of deploying PHRs have been described for both patients and their caregivers. For patients, the access to health data, reliable health-related education and tools for facilitating communication with caregivers and peers, have the potential to empower patients in managing their diseases and to reduce geographical barriers [18, 21]. Providing services for (a)synchronous communication between patient and caregiver may lead to a transition from episodic 'just-in-case' to continuous 'just-in-time' care, which has the potential to shorten the time to address disease-related complaints [15]. Furthermore, caregivers may benefit from more engaged patients as well. A database containing continuous health data from a patient (instead of one snapshot during a consultation) can increase the efficiency of consultations and improve clinical decision making. In turn, this can potentially result in e.g., lower costs for disease management and medication [18].

Despite the potential benefits of PHRs in chronic disease management, PHR research mainly focuses on the barriers for patients or caregivers for using PHRs without taking into account their relation with the context (e.g., [25]). Still, evidence regarding the value of PHRs for selfmanagement remains sparse [26]. Services to support communication between the patient with diabetes and his caregivers are associated with improved glycaemic control [27, 28], but results regarding the effectiveness of other services (e.g., education, insight into disease progression) on improvements in clinical outcomes remain inconclusive [27, 29-33]. This is often due to difficulties with the implementation [34].

In the Netherlands, a growing number of eHealth technologies for chronic disease delivery is already available, but a large-scale adoption is lacking. Information regarding the status of eHealth implementation in the Netherlands is provided in Box 1. 


\section{Box 1. eHealth in the Netherlands}

Since 2012 several (governmental) initiatives have been taken to facilitate the upscaling of technology-based innovations for care delivery. In that year for example, Dutch patients, care providers and health insurance companies have joined their forces to formulate the 'National Implementation Agenda eHealth', containing appointments to support a large-scale implementation of eHealth.

To get annual insight into the availability and use of eHealth technologies by both patients and care providers, the Netherlands institute for health services research (Nivel) and Nictiz, the national competence centre for standardization and eHealth, initiated the 'eHealth Monitor' in 2013. From the first monitor, it could be concluded that there is still a long way to go in order to go to make eHealth successful in the Netherlands, and that there is a need for focus and control within the care field [22].

As a response, the (former) Dutch Minister of Health, Edith Schippers, formulated the following ambitions regarding the use of eHealth in 2014 [23]:

1) Within 5 years, $80 \%$ of all patients with a chronic disease has access to his/her own health information (e.g., information regarding medication, vital functions, lab results) via mobile apps or Internet applications;

2) Within 5 years, $75 \%$ of all frail elderly and chronically ill people is able to perform their own health measurements in order to gain insight into the course of their disease;

3) Within 5 years, every individual who receives home care and support will be able to communicate with a caregiver 24 hours a day via video calling.

Furthermore, the Dutch government and minister Schippers in 2016 committed to provide 20 million euros to stimulate a large-scale implementation of evidence-based eHealth in the next four years.

The results of the eHealth Monitor 2016 [24] indicated that the actual utilization of eHealth tools is still lacking. Many general practitioners (GPs) offer their patients online tools, but only a small number of patients actually uses these tools. Moreover, $64 \%$ of all care consumers does not store any health information, $5 \%$ stores health information on their personal computer, and 32\% stores health information on paper.

One of the main conclusions of this eHealth Monitor was that a large-scale implementation of eHealth asks for a societal innovation, emphasizing human, organizational, and environmental factors. An important recommendation of this report was to facilitate the integration of eHealth into guidelines and care pathways and to train care professionals with regard to this topic. Furthermore, research is needed to develop and identify effective and safe eHealth technologies. 


\section{The evaluation of eHealth}

As stated in Box 1, the availability of results from the evaluation of eHealth technologies is an important governmental precondition for the actual implementation in care processes. At the same time, conclusive evidence on the added value of PHRs remains sparse, which may be due to the used evaluation approaches. Many eHealth technologies (including PHRs) aim to support users in reaching certain health-related behavioural outcomes. Therefore, one of the main goals of eHealth evaluation is to gain insight into the effects of technology on outcomes such as quality of life, health-related outcomes (e.g., glycaemic control, weight loss), or psychological outcomes (e.g., depressive complaints, anxiety) [35]. In these evaluations, technologies are often seen as medical innovations and are therefore evaluated in experimental studies (such as randomized controlled trials (RCTs)) which are the golden standard in medical evaluation research [36]. Although these evaluation approaches have proven to be very useful in determining the effects of many therapies, an increasing group of researchers agree that they do not optimally fit the characteristics of eHealth and therefore have not been able to provide conclusive evidence for different reasons.

First, (quasi) experimental evaluation methods do not provide insight into how, why, and for whom the use of the technology has contributed to the found effects. Using a technology is a dynamic process, resulting in an effect (or not) on daily lives and health conditions. Preand post-measurements however, only provide evidence on e.g., health outcomes, satisfaction, and adoption rates at fixed cut-off points. As a result, information about the interaction process between the user and the technology and how the technology supported the user in healthier living is missing from these evaluations [37]. Health dimensions, costs, usage and other outcome variables related to the context and the process of care delivery are dynamic processes and to gain insight into how these variables change over time, they should preferably be measured continuously.

Second, fundamental to experimental research is to have the technology as a fixed entity for all participants throughout the whole intervention period. After all, when the technology is adjusted during this intervention period, it might remain unclear whether the effects are found despite or thanks to these adjustments. In contrast, (eHealth) technology can be characterized by its constant evolution and when the technology does not move with new developments, apps or interventions have the chance to become obsolete by the time the results of the RCT are available [38].

Third, to accommodate to the complexity of behaviour change, eHealth technologies often consist of multiple components that may interact in reaching a certain effect and that people can use in many different ways in terms of the elements they use as well as the frequency, time and place of use $[39,40]$. The experienced content might therefore differ across all users because technologies are tailored to an individual or because a proportion of the users 
will not be using the technology at all, will stop using the technology after a period of time, or will not use all the available services of the technology. Experimental evaluations however, treat technologies as a singular entity. Again, no insights can be obtained on process outcomes or how the use of the different components of the technology has contributed to healthier living, improved wellbeing, or a user's ability to conduct daily tasks $[37,40,41]$. We call this 'the black box of eHealth' [37, 42].

In Figure 1 this black box is illustrated with an example of the use of an intervention to address mild depressive complaints. The individual in Figure 1a used some of the components of the technology, and after a while, her complaints stayed the same. The individual in Figure $1 \mathrm{~b}$ however, used all the available components and signed up for text message reminders as well. After using the technology for a while, the depressive complaints of this individual decreased significantly. When we just would have looked at the effects of the technology using a (quasi-)experimental design, we would only have been able to see whether the technology was effective or not, without recognizing and acknowledging the differences between the two usage patterns of the individuals in Figure 1a and $1 \mathrm{~b}$.

To open the black box of eHealth and to investigate why, how and for whom a certain technology is of most value in a certain context, methodologies must extend beyond the classic evaluations of effect only [43]. In other words, the characteristics of eHealth technology and the influence of the user and the context in which the technology is implemented, change the way evaluations are conducted. Therefore, it is necessary to look for innovative approaches that go beyond a before and after measurement of health outcomes, for example, by exploring the process by which users find the needed information, share information, and gain benefits out of the eHealth technology. 


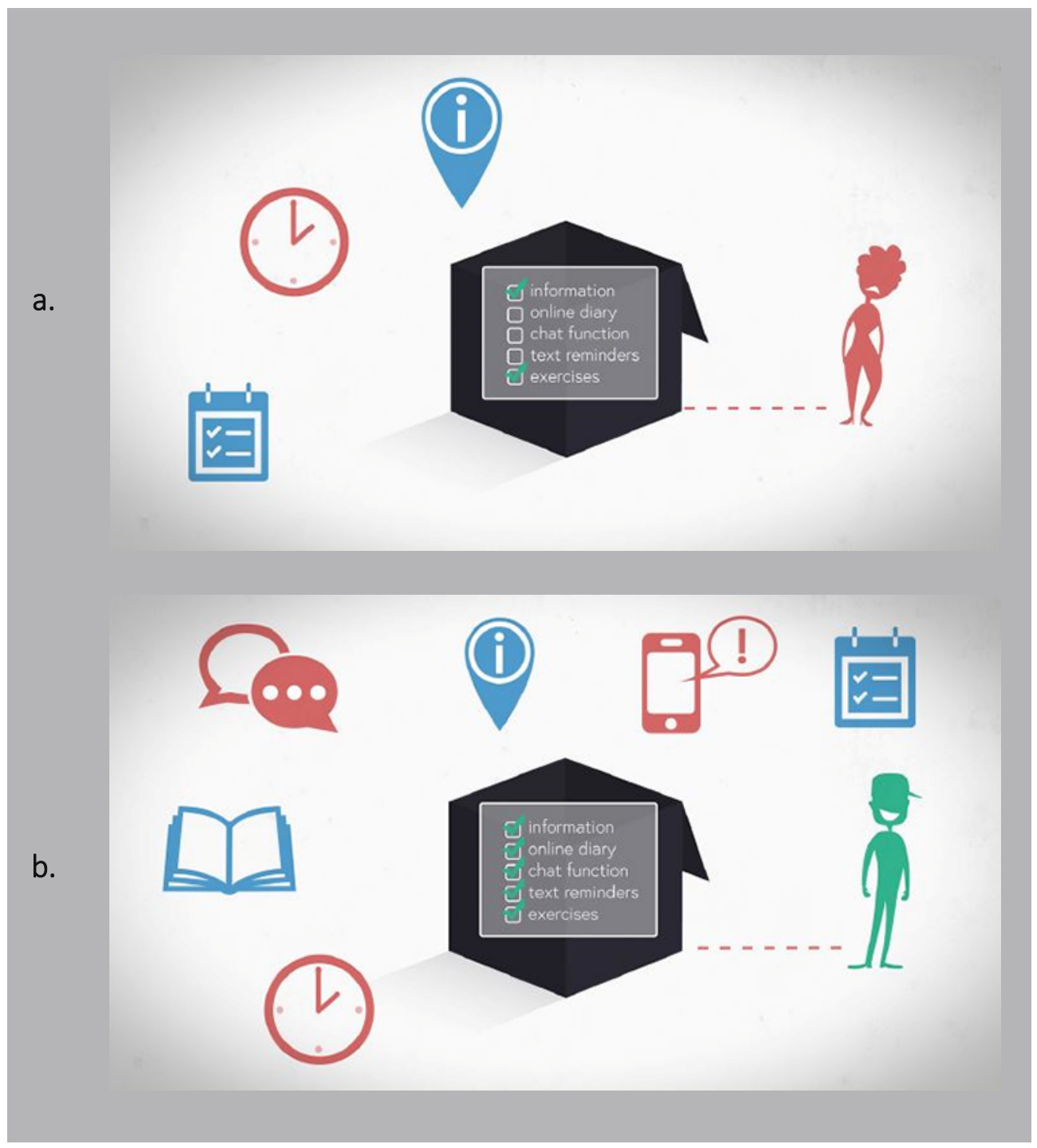

Figure 1. An example of the Black Box of eHealth. Screenshots are retrieved from the massive open online course (MOOC) eHealth: Combining Psychology, Technology and Health (https://www.futurelearn.com/courses/ehealth) 


\section{Personal Health Records for Chronic Care: the Case}

To improve the efficiency and value in a changing care landscape and to stimulate patients with chronic diseases in developing self-management skills, Achmea (a Dutch health insurance company) and Philips Healthcare established the foundation "Care within Reach" (in Dutch: Zorg Binnen Bereik) in 2009. To achieve the objectives, the foundation focused on the development and implementation of the PHR e-Vita.

The concept of the PHR was developed on the basis of experiences of patients and their caregivers and additional brainstorm sessions. The main content of the PHR consisted of health-related education, monitoring health values, and a coach for reaching personal health-related goals. Two first versions of the PHR were developed for type 2 diabetes mellitus (T2DM) and congestive heart failure (CHF) (Figure 2). The PHR for CHF patients was linked to a system for telemonitoring. Patients were asked to monitor their health values via that system on a daily basis, values were then transferred to the PHR. A second version of the PHR was developed for patients with COPD (Figure 3). Screenshots of the different features of the two versions of the PHR can be found on p.169. Detailed information regarding the system and its content can be found in the Chapters 4,5 , and 6 .

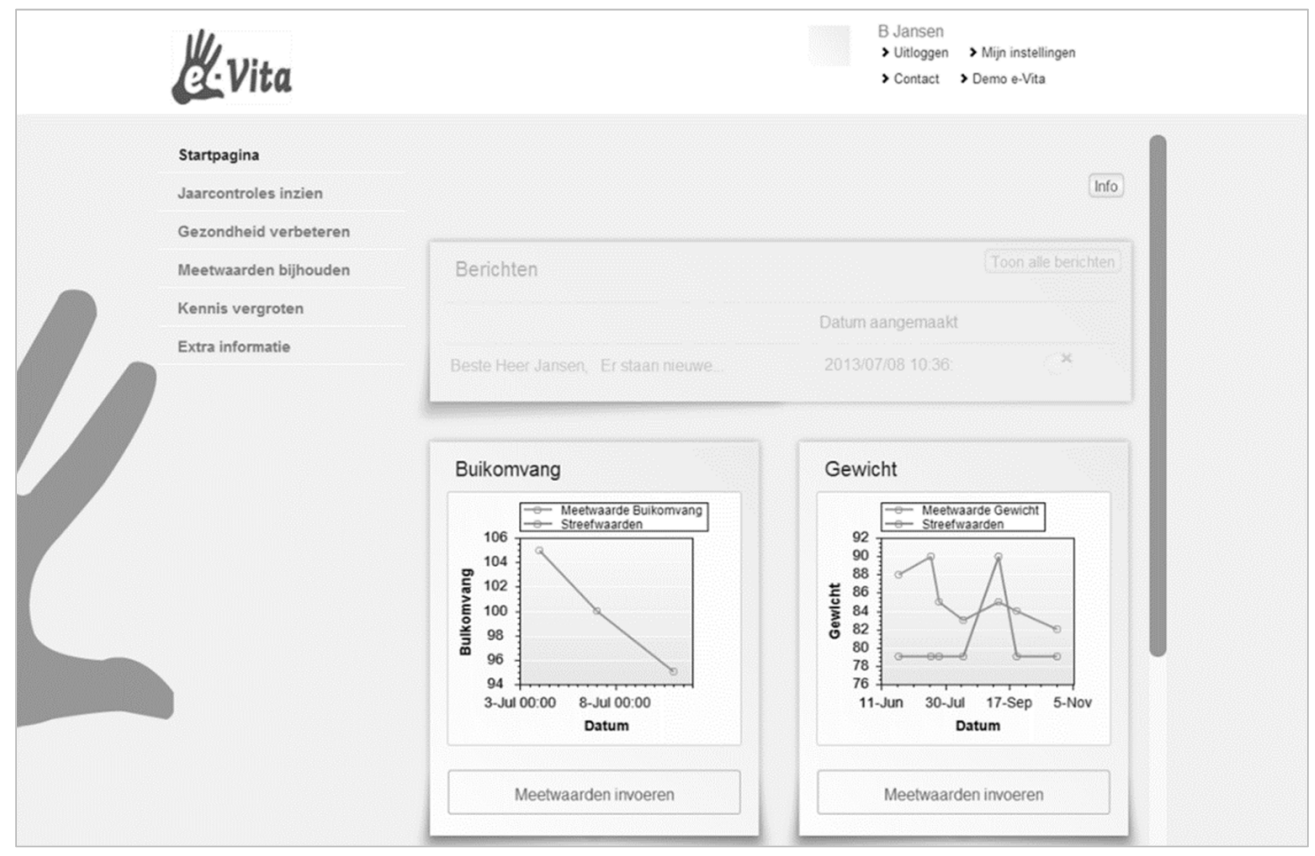

Figure 2. A screenshot of the first version of e-Vita (T2DM / CHF) 


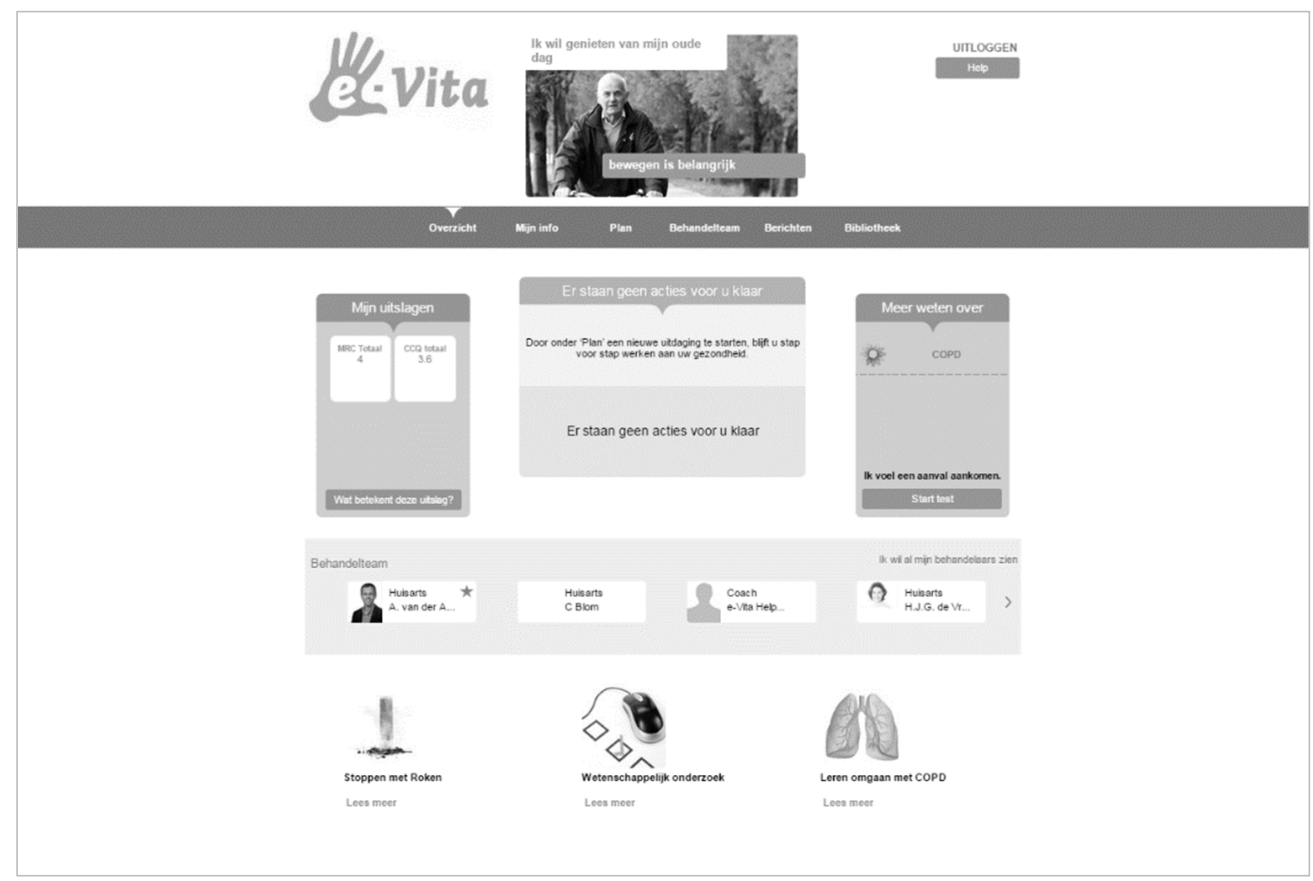

Figure 3. A screenshot of the homepage of the second version of e-Vita (COPD)

The PHRs were implemented in the Netherlands for the duration of three experimental clinical trials to evaluate the effects of the PHR on the quality of life and health outcomes of patients with T2DM [44], CHF [45], and COPD [46]. The T2DM study included as well a fourth randomized controlled trial to evaluate the effectiveness of a coaching module for selfmanagement support for T2DM patients [47]. A fifth research project was conducted to evaluate the impact of the PHR on health care utilization (cost-effectiveness). As described in the previous section, such analyses are valuable, but provide only little insight into why particular outcomes have occurred.

\section{This Thesis}

To understand what differences PHRs can make in health care, why PHRs make these differences, and why PHRs may or may not have the expected impact, a sixth study was conducted within the e-Vita project. This study on the implementation of PHRs incorporates all three versions of e-Vita and is the topic of this thesis. The research questions that are used as a guidance in this project are presented in Box 2. Several of these research questions refer to the stakeholders in the project. Although this term can refer to all people with an interest (e.g., management, health care insurance companies), in this thesis we focus on the endusers of e-Vita: patients with T2DM, CHF, or COPD and their caregivers. To be more specific, we focus on these stakeholders and how they use the PHR in the context of their care 
(patients) or working routines (caregivers). To the best of our knowledge, this evaluation of a PHR incorporating different perspectives in a mixed methods approach is unique.

Box 2. Research questions of this thesis

1. How is the e-Vita PHR used on the long-term by its stakeholders?

a. To what extent is the PHR used by its stakeholders on the long-term?

b. What usage patterns emerge?

c. What is the adherence rate?

d. What services on the PHR are used?

2. Who are the intended and actual users of the e-Vita platform?
a. Who are the hard-core users?
b. Who are the low-users, and drop outs?
c. What user profiles can be identified?

3. Is the e-Vita PHR perceived as user-friendly?

a. Does the PHR provide the services the user is looking for?

b. Is the PHR easy to use?

c. Is the information provided via the PHR understandable and reliable?

d. Does the PHR fit the users' preferences?

e. Is the PHR interoperable with other systems?

4. Are the stakeholders satisfied with the provided service via the PHR?

a. What are the net benefits, according to the stakeholders?

b. How supportive is the PHR in providing self-management support and feedback?

5. What implementation scenarios can be developed for the integration of the PHR in healthcare processes to realize its added value?

The assumptions of the CeHRes Roadmap for the development and evaluation of eHealth technologies (Figure 4) were used as the basis for our research. The roadmap states that eHealth development is a participatory process that is intertwined with the implementation into daily (health care) routines. Within this process, the development and evaluation of technology is an iterative, flexible and dynamic process without a fixed endpoint. This requires formative and summative evaluations that are interwoven with all stages of technology development. 


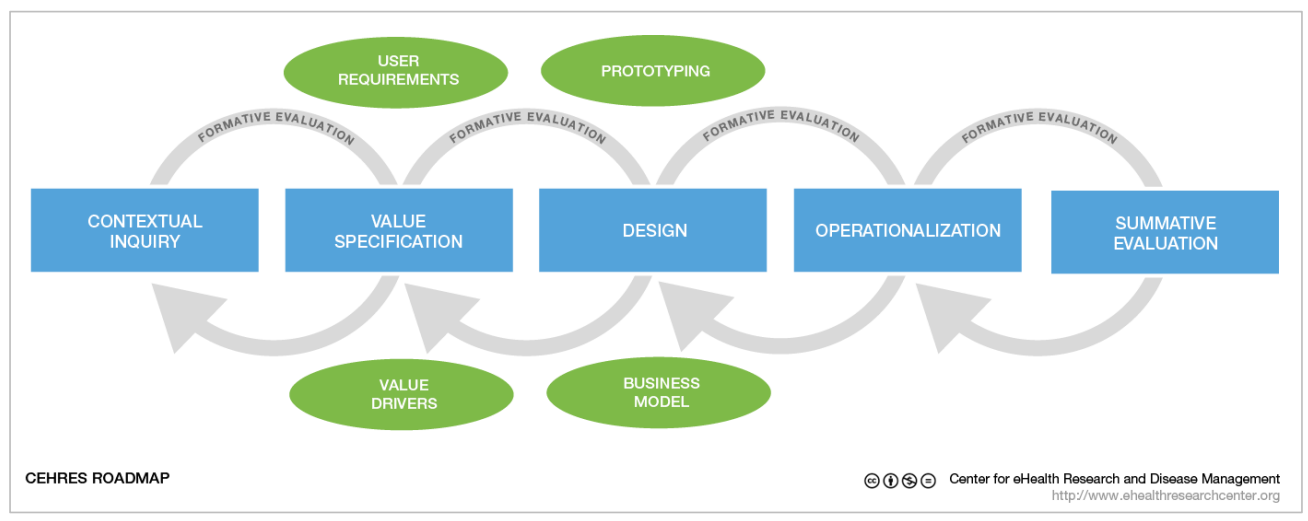

Figure 4. The CeHRes Roadmap

\section{Part 1 - Conceptual Framework}

Implementation implies that the technology is used and in this thesis, we therefore consider technology usage as a proxy for the implementation. In eHealth research, technology usage is often used as a measure for adherence. However, there is a lot of ambiguity about how adherence should be defined for technology that can be used in many different ways and for many different target groups. Therefore, the first part of this thesis consists of two more fundamental chapters as a framework for the evaluation. Chapter 2 of this thesis describes a systematic review to gain more insight into the concepts of adherence and intended use. Chapter 3 describes our approach for the analysis of the usage data; the log data analysis.

\section{Adherence and intended use}

The term adherence is rooted in pharmaceutical industry and according to the definition of the WHO, it refers to "the extent to which a person's behaviour -taking medication, following a diet, and/or executing lifestyle changes, corresponds with agreed recommendations from a health care provider" [48]. In this field, the intended usage (i.e. agreed recommendations) is mostly based on the observed or reasoned working mechanisms and the dose response curves of the medication for a certain condition. As a result, the dosage of one particular medication can vary depending on the (severity of) the condition and the characteristics of the patient (e.g., age, gender, or weight) $[39,40]$.

This is in contrast with many prior eHealth studies, where it is often assumed that all users should experience all the elements of a technology to gain effects, and where adherence is thus often operationalized as using everything the technology offers [49, 50]. However, the PHR e-Vita was designed for multiple target groups and, dependent of the individual user goals and the desired outcomes (that could be the result of a shared decision making process [7]), it can be used in many different ways in terms of the features that are used, as well as the frequency, time, and place of use. This implies that individuals do not always have to use 
all the available elements or have to use the same elements. Moreover, individuals may stop using (certain features of) the technology because they have reached their personal goals (early completers or e-attainers [51]), and non-usage dropout is thus not always a consequence of losing interest. Therefore, Chapter 2 describes a systematic review to clarify the concept of adherence and to find a concise way to operationalize adherence to eHealth technologies.

\section{Log data}

The analysis of log data can provide continuous and objective insights into the actual usage of and adherence to the technology [41]. Up to now, log data analyses in eHealth research have mainly focused on descriptive statistics, such as the number of logins, time spent and the frequency of use of the different elements by all users as a group. Although these statistics do provide valuable information regarding the usage of the technology, they also assume that more use is always better, without taking the goal of the user into account. Furthermore, such analyses do not always provide insight into the actual process of technology use in relation to behaviour change. To understand the potential and added value of log data analyses in eHealth evaluations, Chapter 3 describes a protocol for log data analyses of eHealth technology. Using iterative, flexible and dynamic evaluation cycles, the outcomes of such log data evaluations can be used for a process analysis, recognizing the areas of improvement and diving deeper into the concepts of adherence and the usage (the dose) that is needed to reach certain effects (the response).

\section{Part 2 - The evaluation of the implementation of e-Vita}

The second part of this thesis contains three chapters to gain insight into the actual implementation of e-Vita, the relation between the user and the technology and the influence of the context. As stated before, creating sustainable eHealth technologies requires a holistic approach that takes into account the triad between the technology, its users and the context of implementation. This implies that a good understanding regarding the functioning of technology in a certain context can seldom be obtained by conducting a single evaluation method from one point of view.

Because previous research showed that users are likely to drop out when they 'get lost' in the intervention, the first impression of a technology is of great importance. To gain insight into how users explore a new technology, Chapter 4 describes a log data analysis of the first use of e-Vita T2DM, from the perspective of the patient. To gain insight into the experiences of care providers regarding the implementation and the perceived added value of the PHR, Chapter 5 describes an interview study. Chapter 6 combines these different perspectives in a mixed method evaluation. Qualitative and quantitative data sources are used to gain insight into how the use of the PHR was influenced by the implementation process and the interaction between the patient and the caregiver. Based on the findings, lessons learned for 
the evaluation and implementation of eHealth technology will be discussed in Chapter 7, the general discussion of this thesis. 


\section{References}

1. Timmreck, T.C., Dictionary of health services management. 1987: National Health Pub.

2. World Health Organization, Global status report on noncommunicable diseases 2014. 2014: World Health Organization.

3. World Health Organization, Noncommunicable Diseases (NCD) Country Profiles for the Netherlands. 2014. Available from: http://www.who.int/nmh/countries/nld_en.pdf?ua=1.

4. Baan, C.A. and M.J.J.C. Poos. Diabetes mellitus - Cijfers en Context - Huidige Situatie. Available from:https://www.volksgezondheidenzorg.info/onderwerp/diabetes-mellitus/cijferscontext/huidige-situatie.

5. Engelfriet, P.M., M.J.J.C. Poos, and F.H. Rutten. Hartfalen - Cijfers en Context - Huidige Situatie. Available from: https://www.volksgezondheidenzorg.info/onderwerp/hartfalen/cijferscontext/huidige-situatie.

6. Poos, M.J.J.C. and M. Nielen. COPD - Cijfers en Context - Huidige Situatie. Available from: https://www.volksgezondheidenzorg.info/onderwerp/copd/cijfers-context/huidige-situatie.

7. Roehrs, A., et al., Personal Health Records: A Systematic Literature Review. J Med Internet Res, 2017. 19(1): p. e13

8. Wagner, E.H., et al., Improving Chronic Illness Care: Translating Evidence Into Action. Health Affairs, 2001. 20(6): p. 64-78.

9. World Health Organization. Constitution of the World Health Organization. 2006; Available from: http://www.who.int/governance/eb/who_constitution_en.pdf.

10. Huber, M., et al., How should we define health? BMJ, 2011. 343.

11. World Health Organization, Innovative care for chronic conditions: building blocks for actions: global report. 2002.

12. Bodenheimer, T., et al., Patient self-management of chronic disease in primary care. JAMA, 2002. 288(19): p. 2469-2475.

13. Task Force on Community Preventive Services, Recommendations for healthcare system and selfmanagement education interventions to reduce morbidity and mortality from diabetes. American Journal of Preventive Medicine, 2002. 22(4): p. 10-14.

14. Campbell, H., et al., Integrated care pathways. BMJ : British Medical Journal, 1998. 316(7125): p. 133-137.

15. Verhoeven, F., et al., Asynchronous and Synchronous Teleconsultation for Diabetes Care: A Systematic Literature Review. Journal of Diabetes Science and Technology, 2010. 4(3): p. 666-684.

16. Eysenbach, G., What is e-health? J Med Internet Res, 2001. 3(2): p. e20.

17. Nazi, K.M., The Personal Health Record Paradox: Health Care Professionals' Perspectives and the Information Ecology of Personal Health Record Systems in Organizational and Clinical Settings. J Med Internet Res, 2013. 15(4): p. e70.

18. Tang, P.C., et al., Personal Health Records: Definitions, Benefits, and Strategies for Overcoming Barriers to Adoption. Journal of the American Medical Informatics Association, 2006. 13(2): p. 121126.

19. Otte-Trojel, T., et al., How outcomes are achieved through patient portals: a realist review. Journal of the American Medical Informatics Association, 2014. 21(4): p. 751-757. 
20. Markle Connecting for Health. The Personal Health Working Group final report. July 1, 2003 Retrieved from: https://www.markle.org/publications/1429-personal-health-working-group-finalreport.

21. Pagliari, C., D. Detmer, and P. Singleton, Potential of electronic personal health records. BMJ, 2007. 335(7615): p. 330-333.

22. Nictiz and Nivel, eHealth, verder dan je denkt. eHealth-monitor 2013. 2013.

23. Schippers, E.I. and M.J.van Rijn, Kamerbrief over e-health en zorgverbetering. 2014: The Hague.

24. Nictiz and Nivel, Meer dan techniek. eHealth Monitor 2016. 2016.

25. Showell, C., Barriers to the use of personal health records by patients: a structured review. PeerJ, 2017. 5: p. e3268

26. Johansen, M.A. and E. Henriksen, The Evolution of Personal Health Records and their Role for SelfManagement: A Literature Review, in Studies in Health Technology and Informatics. 2014. p. 458462.

27. Coughlin, S.S., L.B. Williams, and C. Hatzigeorgiou, A systematic review of studies of web portals for patients with diabetes mellitus. mHealth, 2017. 3(6).

28. Goldzweig, C.L., et al., Secure Messaging between Providers and Patients, and Patients' Access to Their Own Medical Record. 2012.

29. Osborn, C.Y., et al., Patient web portals to improve diabetes outcomes: A systematic review. Current Diabetes Reports, 2010. 10(6): p. 422-435.

30. Kooij, L., W.G. Groen, and W.H. Van Harten, The effectiveness of information technology-supported shared care for patients with chronic disease: A systematic review. Journal of Medical Internet Research, 2017. 19(6).

31. Giardina, T.D., et al., Patient access to medical records and healthcare outcomes: A systematic review. Journal of the American Medical Informatics Association, 2014. 21(4): p. 737-741.

32. Goldzweig, C.J., et al., Electronic Patient Portals: Evidence on Health Outcomes, Satisfaction, Efficiency, and Attitudes - A Systematic Review. Annals of Internal Medicine, 2013. 159(10): p. 677687.

33. Tenforde, M., A. Jain, and J. Hickner, The value of personal health records for chronic disease management: what do we know? Family Medicine-Kansas City, 2011. 43(5): p. 351.

34. Ross, J., et al., Factors that influence the implementation of e-health: a systematic review of systematic reviews (an update). Implementation Science, 2016. 11(1): p. 146.

35. Glasgow, R.E., eHealth Evaluation and Dissemination Research. American Journal of Preventive Medicine, 2007. 32(5): p. S119-S126.

36. Eysenbach, G., CONSORT-EHEALTH: Improving and Standardizing Evaluation Reports of Web-based and Mobile Health Interventions. J Med Internet Res, 2011. 13(4): p. e126.

37. Resnicow, K., et al., Methodologic and Design Issues in Patient-Centered e-Health Research. American Journal of Preventive Medicine, 2010. 38(1): p. 98-102.

38. Glasgow, R.E., S.M. Phillips, and M.A. Sanchez, Implementation science approaches for integrating eHealth research into practice and policy. International Journal of Medical Informatics, 2014. 83(7): p. e1-e11.

39. Moller, A.C., et al., Applying and advancing behavior change theories and techniques in the context of a digital health revolution: proposals for more effectively realizing untapped potential. Journal of Behavioral Medicine, 2017. 40(1): p. 85-98. 
40. Hekler, E.B., et al., Agile science: creating useful products for behavior change in the real world. Translational Behavioral Medicine, 2016. 6(2): p. 317-328.

41. Han, J.Y., Transaction logfile analysis in health communication research: Challenges and opportunities. Patient Education and Counseling, 2011. 82(3): p. 307-312.

42. Black, A.D., et al., The Impact of eHealth on the Quality and Safety of Health Care: A Systematic Overview. PLOS Medicine, 2011. 8(1): p. e1000387.

43. Damschroder, L.J., et al., Fostering implementation of health services research findings into practice: a consolidated framework for advancing implementation science. Implementation Science, 2009. 4(1): p. 50.

44. Roelofsen, Y., et al., Design of the e-Vita diabetes mellitus study: effects and use of an interactive online care platform in patients with type 2 diabetes (e-VitaDM-1/ZODIAC-40). BMC Endocrine Disorders, 2014. 14(1): p. 22.

45. Wagenaar, K.P., et al., Effectiveness of an interactive platform, and the ESC/HFA heartfailurematters.org website in patients with heart failure: design of the multicentre randomized e-Vita heart failure trial. European Journal of Heart Failure, 2015. 17(12): p. 1310-1316.

46. Talboom-Kamp, E.P.W.A., et al., e-Vita: design of an innovative approach to COPD disease management in primary care through eHealth application. BMC Pulmonary Medicine, 2016. 16(1): p. 121.

47. van Vugt, M., et al., Web-based self-management with and without coaching for type 2 diabetes patients in primary care: design of a randomized controlled trial. BMC Endocrine Disorders, 2013. 13(1): p. 53.

48. Sabaté, E., Adherence to long-term therapies: evidence for action. 2003: World Health Organization.

49. Donkin, L., et al., A Systematic Review of the Impact of Adherence on the Effectiveness of eTherapies. J Med Internet Res, 2011. 13(3): p. e52.

50. Christensen, H., K.M. Griffiths, and L. Farrer, Adherence in Internet Interventions for Anxiety and Depression: Systematic Review. J Med Internet Res, 2009. 11(2): p. e13.

51. Christensen, H. and A. Mackinnon, The Law of Attrition Revisited. J Med Internet Res, 2006. 8(3): p. e20. 

Part 1

Conceptual Framework 



\section{Chapter 2}

When does usage become adherence?

A systematic review to clarify the concept of adherence to eHealth technology

F. Sieverink, S. Kelders, J. van Gemert-Pijnen Journal of Medical Internet Research (Accepted) 


\section{Abstract}

Background: In eHealth evaluations, there is increasing attention for reporting the actual usage of a technology in relation to the outcomes found. This is often done by studying the adherence to the technology. Based on the definition of adherence, we suggest that three elements are necessary to determine adherence to eHealth technology: 1) the ability to measure the usage behavior of individuals; 2 ) an operationalization of intended use; and 3) an empirical, theoretical, or rational justification of the intended use. However, little is known to this day about how to operationalize the intended usage of and the adherence to different types of eHealth technology.

Objectives: The aim of this systematic review is to improve eHealth evaluations by 1) gaining insight into when, how, and by whom the concept of adherence has been used in previous eHealth evaluations; and 2) finding a concise way to operationalize adherence to and intended use of different eHealth technologies.

Methods: A systematic review of eHealth evaluations was conducted to gain insight into how the use of the technology was measured, how adherence to different types of technologies was operationalized, and if and how the intended use of the technology was justified. Differences in variables between the use of the technology and the operationalization of adherence were calculated using a Chi-square test of independence.

Results: In total, 62 studies were included in this review. In 34 studies, adherence was operationalized as 'the more use, the better', while 28 studies described a threshold for intended use of the technology as well. Out of these 28 , only 6 reported a justification for the intended use. The proportion of evaluations of mental health technologies reporting a justified operationalization of intended use is lagging behind compared to evaluations of lifestyle and chronic care technologies. The results indicated that a justification of intended use does not require extra measurements to determine adherence to the technology.

Conclusion: The results of this review showed that the operationalization of adherence is often based on the assumption of 'the more use, the better' and justifications for intended use are often missing. Obviously, it is not always possible to estimate the intended use of a technology. However, since evaluating adherence requires an operationalization of intended use, such measures do not meet the definition of adherence and should therefore be referred to as the actual usage of the technology. Therefore, it can be concluded that adherence to eHealth technology is an underdeveloped and often improperly used concept in the existing body of literature.

When defining the intended use of a technology and selecting valid measures for adherence, the goal and/or the assumed working mechanisms should be leading. Adherence can then 
be standardized, which will improve the comparison of adherence rates to different technologies with the same goal, and will provide insight into how adherence to different elements contributed to the outcomes.

Keywords: Adherence, intended use, eHealth, systematic review 


\section{Introduction}

One of the main goals of eHealth evaluations is to gain insight into the effects of the technology on outcomes such as quality of life, health-related outcomes (e.g., glycemic control, weight loss), or psychological outcomes (e.g., depressive complaints, anxiety). However, many eHealth evaluations report no or limited positive effects [1-5]. There is strong evidence that this is often related to participants not using technologies in the desired way. For every technology, a proportion of the users will not use the intervention at all, will stop using the technology after a period of time, or will not use the available elements of the technology as intended [1, 6-8].

To gain more insight into this phenomenon, Eysenbach made a plea back in 2005 for reporting the levels of non-usage attrition, or the extent to which individuals stop using the technology [9]. On the other hand, understanding adherence, or how actual usage of the technology may have influenced the outcomes, might be just as important [6]. The term adherence is rooted in the pharmaceutical industry and according to the WHO's definition, it refers to "the extent to which a person's behaviour - taking medication, following a diet, and/or executing lifestyle changes, corresponds with agreed recommendations from a health care provider" [10].

For eHealth technologies, several definitions for adherence can be identified in the existing literature. For example, Christensen et al. defined adherence as "the degree to which individuals experience the content of the Internet intervention" [11]. However, the concept of 'following the prescribed recommendations' (as implied by the WHO's definition) is missing from this definition. Therefore, Donkin et al. referred to adherence as "the degree to which the user followed the program as it was designed" [6]. In accordance with the WHO definition of adherence, this definition contains the concept of intended use, or "the extent to which individuals should experience the content to derive maximum benefit from the intervention, as defined or implied by its creators" [1]. According to these definitions, the intended use is thus the minimum use to establish adherence.

Although adherence is related to other measures such as engagement or non-usage attrition, these terms do not refer to the same or inverse concepts. After all, not using the technology as defined or implied by its creators does not necessarily mean that a participant is not using the technology at all (as implied by the definition of non-usage attrition [9]). Moreover, definitions of engagement usually incorporate the more subjective attributes of challenge, positive affect, endurability, aesthetic and sensory appeals [12], while adherence is mostly based on measures for usage behavior.

There is now increasing attention for studying the adherence rates and reasons for nonadherence in eHealth evaluations. However, it still can be a challenge to operationalize the 
intended use for individual eHealth technologies in a certain context. In the pharmaceutical industry, the intended use (i.e. agreed recommendations) is mostly based on the observed or rationalized working mechanisms and the dose-response curves of the medication for a certain condition. As a result, the dosage of one particular medication can vary depending on (the severity of) the condition and the patient's characteristics (e.g., age, gender, or weight).

This is in contrast with many prior eHealth studies, which often assume that all users should experience all of the elements of a technology to obtain effects, and in which adherence is thus often operationalized as using everything the technology offers. However, a technology can be designed for multiple target groups and, depending on the individual user goals and the desired outcomes, technology can be used in many different ways in terms of the features that are used, as well as the frequency, time, and place of use $[13,14]$. Furthermore, the amount of use that is needed to obtain the desired outcomes may vary a lot across different user groups [6]. This implies that users do not always have to experience all of the available elements of a technology or have to use the same elements, since usage goals may differ across users as well. Moreover, individuals may also stop using the technology because they have reached their personal goals (early completers or e-attainers) [11, 15] and nonusage dropout is thus not always a consequence of losing interest (as stated by Eysenbach [9]).

To summarize, based on the definition of adherence, we suggest that three elements are necessary to determine adherence to eHealth technology: 1) the ability to measure the usage behavior of individuals; 2) an operationalization of intended use; and 3) an empirical, theoretical, or rational justification of the intended use. However, little is known to this day about how to operationalize the intended usage of and thus the adherence to different types of eHealth technology. Many systematic reviews gaining insight into adherence to eHealth technology focus on the extent to which individuals use different types of technology and what the reasons for non-adherence are, without a proper operationalization of intended use and adherence $[1,6,11,16,17]$. These reviews therefore fail to provide insight into how adherence and intended use have been operationalized.

The goal of this systematic review is therefore to improve evaluations of eHealth technologies by 1) gaining insight into when, by whom, and how, the concept of adherence has been used in previous eHealth evaluations; and 2) finding a concise way to operationalize the adherence to and the intended use of different eHealth technologies. We do this by providing insight into how the usage of the technology was measured across previous studies; how adherence to different types of technologies (e.g., structured interventions, patients platforms) was operationalized; and if and how the intended use of eHealth technologies has been justified with theory, evidence or rationale. 


\section{Methods}

\section{Search strategy}

A literature search was conducted using the Scopus, Web of Science, ScienceDirect and PsycINFO databases. A combination of the constructs 'technology', 'intervention', 'adherence' and 'health' was used. To ensure sufficient coverage of each construct, we used different keywords for every construct (see Multimedia Appendix 1). We excluded other usage-related concepts (e.g., non-usage attrition or engagement) because these do not refer to the same concept.

\section{Eligibility Criteria}

All articles that met the following criteria were included in the review: 1) it involved healthrelated technology (web-based technologies, apps, wearables or technologies provided via other devices); 2) the technology was intended to be used more than once by the patient or client; 3) the article described a (protocol for a) primary study that included objective, quantifiable measurements and an operationalization of adherence to the technology; 4) the study was published in English; and 5) the study was peer-reviewed and published.

Articles were excluded in the following situations: 1 ) adherence was defined as adhering to offline treatment or as a measure for following a study protocol; 2) the technology studied was only used as a tool for exchanging information without the possibility for further interaction with the system (e.g. telemonitoring only, sending or receiving messages like SMS interventions or in chat rooms); and 3) the article was a conference abstract or a full-text was not available.

\section{Study Selection}

The selection of studies was completed in three steps. First, all titles were screened by two authors (FS and SK) to exclude the records that clearly indicated a study outside the scope of this review (e.g. medication adherence). Second, the abstracts of the articles initially deemed relevant were screened for eligibility by those same authors. During this process of title and abstract screening, studies were included in the next step if they were deemed eligible by at least one of the reviewers.

Third, the full texts of all remaining publications were checked for inclusion by FS, and the final selection was discussed by FS, SK, and LVG. Disagreements regarding the inclusion of full texts were discussed until consensus was reached. 


\section{Data Collection and analysis}

The required information for all included technologies and studies was coded by FS using a data extraction form. The information that was extracted from each article is presented in Box 1.

Box 1. Information extracted from the included articles.

1. General information regarding the authors, affiliation, country, year, and journal of publication.

2. The name of the technology. When no name was reported, the name was indicated as 'n/a'.

3. The type of technology, or the device. For example, web-based, smartphone app, wearable, or other devices for monitoring.

4. Type of use (structured, hybrid, or unstructured). 'Structured use' was assigned to technologies consisting entirely of separate modules or lessons that users had to complete prior to moving on to the next [6]. 'Free use' was assigned to technologies that consisted of different elements that users could then use at their own convenience (e.g. a personal health record containing a diary, educational material, and a messaging function; or a wearable connected to a mobile phone app to gain insight into something like activity levels). 'Hybrid use' was assigned to technologies with a fixed core, supplemented with other components for free use.

5. The healthcare field targeted with the technology, distinguishing between mental health (e.g. targeting depressive symptoms or anxiety), chronic conditions (e.g. selfmanagement support for patients with type 1 diabetes mellitus) or lifestyle technologies (e.g. losing weight, improving physical activity, quiting smoking). These categories were assigned depending on the technology's goal, meaning that an intervention to support patients with chronic conditions maintaining a healthy lifestyle is seen as a lifestyle technology.

6. The variables that were used to assess adherence, such as the number of logins, the number of different days that users used the technology, the time spent on the technology, the number of modules or lessons started or completed, and the number of different elements that were accessed or used.

7. The intended use of the technology

8. Whether the described intended use was justified, for example using theory, evidence or rationale.

Based on the extracted information, the operationalizations for adherence in every study were categorized. An overview of these categories is provided in Table 1. 
Table 1. Categorization of adherence operationalizations

\begin{tabular}{|l|l|}
\hline Category & Explanation \\
\hline Category A & $\begin{array}{l}\text { Assigned when adherence was operationalized in terms of 'the more } \\
\text { usage, the better'. Category A operationalizations do not include an } \\
\text { operationalization of intended use, and do therefore not comply with } \\
\text { the definition of adherence. }\end{array}$ \\
\hline Category B & $\begin{array}{l}\text { Assigned when the intended use of a technology was provided } \\
\text { without justification (e.g., 'a user is adherent when logging in at least } \\
\text { once a week for three subsequent weeks'). }\end{array}$ \\
\hline Category C & $\begin{array}{l}\text { Assigned when the intended use of the technology was provided and } \\
\text { justified using theory, evidence or rationale (e.g., 'we know from } \\
\text { previous research that users benefit the most from the technology } \\
\text { when finishing module 4, so a user is adherent once module 4 is } \\
\text { completed'). }\end{array}$ \\
\hline
\end{tabular}

All of the data on each study was entered in SPSS version 24.0 (IBM Corporation, Somers, NY, USA). Each was treated as a separate case. The results are categorized based on the use of the technology (structured, hybrid, and unstructured) and the categorization of adherence operationalizations (Category A, B, and C). Descriptive data for the different categories was calculated using SPSS. Differences in variables between the use of the technology and the operationalization of adherence were calculated using a chi-square test of independence. When the observed counts were below the expected counts, a Monte Carlo correction was applied. We used an alpha level of .05 for all statistical tests.

\section{Results}

\section{Study Selection}

A total of 7,005 studies were identified via the search. After screening of the titles, abstracts and full texts, 62 full texts were included in this review. An overview of these articles is presented in Multimedia Appendix 2.

In total, 36 articles were excluded during the full-text screening phase (Figure 1). Most full texts $(n=18)$ were excluded because they did not include objective, quantifiable measurements and an operationalization of adherence to the technology (12 primary studies and 6 viewpoint papers). Other reasons for exclusion are presented in Figure 1. 


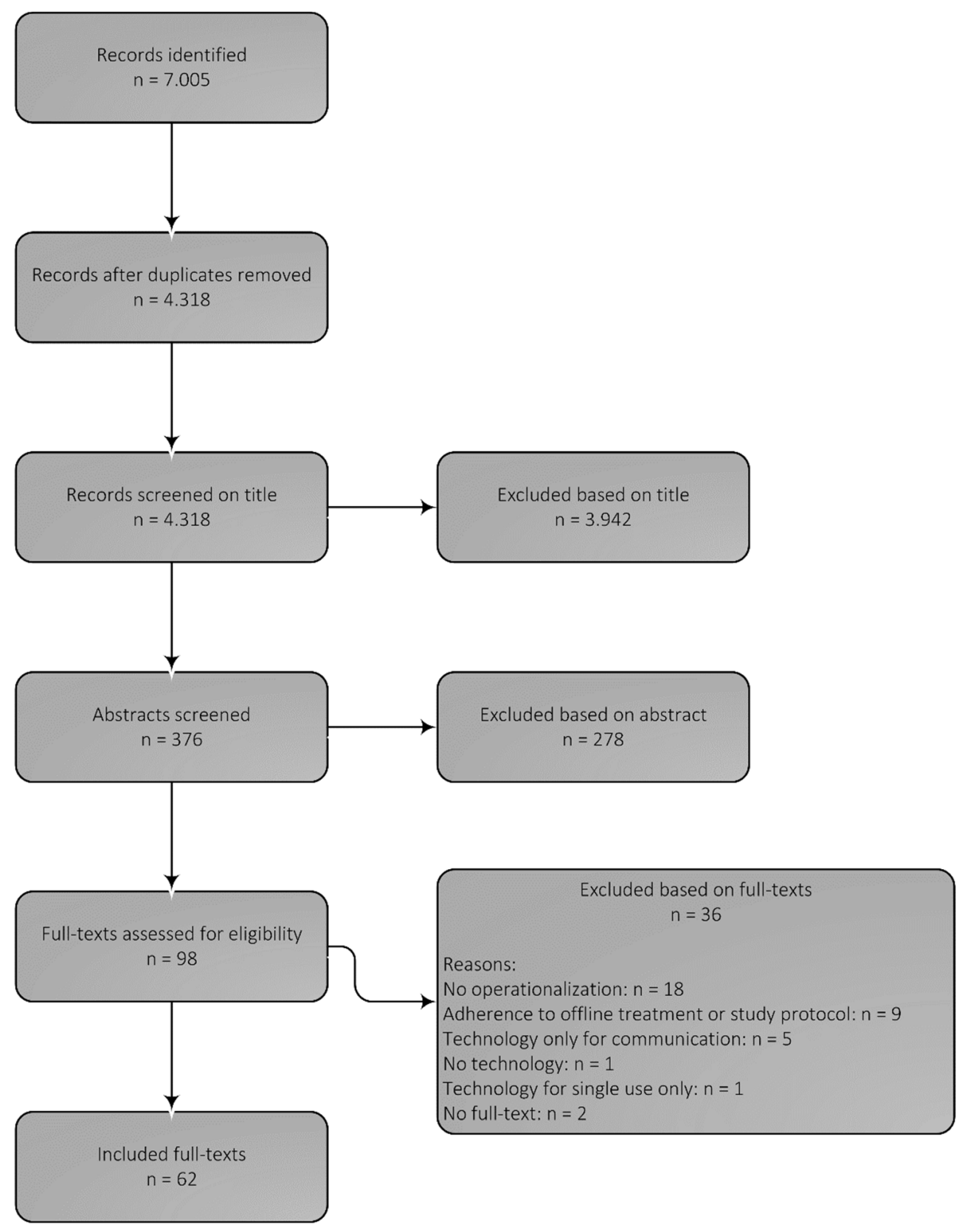

Figure 1. Flowchart of full-text selection 
All included articles were published in 2006 or later, and more articles published in recent years were included overall (Figure 2). The first authors are mostly affiliated in the United States of America ( $n=15)$, Australia $(n=10)$, and the Netherlands ( $n=8)$ (Table 2). In total, 24 of the studies were published in the Journal of Medical Internet Research or its sister journals.

Table 2. Country of affiliation of the first authors of all included articles.

\begin{tabular}{|l|c|}
\hline Country & $\begin{array}{c}\text { Number of } \\
\text { included articles }\end{array}$ \\
\hline United States of America & 15 \\
\hline Australia & 10 \\
\hline The Netherlands & 8 \\
\hline Sweden & 5 \\
\hline United Kingdom & 5 \\
\hline Canada & 3 \\
\hline Germany & 3 \\
\hline Switzerland & 3 \\
\hline China & 2 \\
\hline Norway & 2 \\
\hline Austria & 1 \\
\hline Denmark & 1 \\
\hline Finland & 1 \\
\hline Ireland & 1 \\
\hline Portugal & 1 \\
\hline Spain & 1 \\
\hline
\end{tabular}

\section{Technology characteristics}

Table 3 provides an overview of the technologies that are the subjects of the included studies. The technologies described in most of the articles are web-based (51/62). Furthermore, five are smartphone apps and five are web-based or smartphone technologies combined with other devices such as wearables. Almost half of the technologies (29/62) were structured technologies, 18 were unstructured technologies, and 15 had a hybrid nature.

Half of all included articles reported adherence to mental health technologies. Most of these technologies targeted depressions $(n=8)$ and anxiety disorders $(n=5)$, some of the latter also in combination with depressions $(n=3)$. Other mental health technologies targeted postdisaster mental health distress $(n=3)$; cancer-related distress $(n=3)$; general stress management $(n=2)$; eating pathology $(n=2)$; or insomnia, erectile dysfunction, bipolar disorders, mindfulness, and cognitive training (all $n=1$ ). Eighteen of these technologies were 
based on cognitive behavioral therapy. Most of the structured technologies (17/29) and almost all hybrid technologies $(12 / 15)$ were aimed at improving mental health $(p<.001)$.

Twenty-five technologies were aimed at supporting a healthy lifestyle, more specifically smoking cessation $(n=7)$; improving physical activity $(n=7)$; weight loss $(n=5)$; alcohol cessation ( $n=3)$; general health promotion $(n=2)$; or healthy eating $(n=1)$. Six technologies were aimed at self-management support for patients with chronic diseases (diabetes $(n=3)$, inflammatory bowel disease $(n=1)$, hypertension $(n=1)$ or surgical site infections $(n=1))$. Most of the unstructured technologies were aimed at lifestyle support (13/18).

For all technologies, adherence was mostly operationalized using measures regarding the number of modules or lessons completed and the number of different days, weeks, or months people used the technology. Adherence to unstructured technologies was mostly operationalized using the number of logins or sessions $(p=.03)$, the number of features accessed or used $(p<.001)$, and the time spent using the technology $(p<.001)$. Adherence to structured technologies was most often operationalized using the number of modules or lessons completed $(p<.001)$. 
Table 3. Characteristics of structured, unstructured and hybrid technologies and their operationalizations of adherence

\begin{tabular}{|c|c|c|c|}
\hline & $\begin{array}{c}\text { Structured } \\
\qquad \begin{array}{c}(n=29) \\
n(\%)\end{array}\end{array}$ & $\begin{array}{l}\text { Unstructured } \\
\qquad \begin{array}{c}(n=18) \\
n(\%)\end{array}\end{array}$ & $\begin{array}{c}\text { Hybrid } \\
(n=15) \\
n(\%)\end{array}$ \\
\hline \multicolumn{4}{|l|}{ Healthcare field ${ }^{*}$} \\
\hline Mental health $(n=31)$ & $17(59)$ & $2(11)$ & $12(80)$ \\
\hline Lifestyle $(n=25)$ & $9(31)$ & $13(72)$ & $3(20)$ \\
\hline Chronic care $(n=6)$ & $3(10)$ & $3(17)$ & $0(0)$ \\
\hline \multicolumn{4}{|l|}{ Device } \\
\hline Web-based $(n=51)$ & $25(86)$ & $13(72)$ & $13(87)$ \\
\hline Smartphone app $(n=6)$ & $3(10)$ & $2(11)$ & $1(7)$ \\
\hline $\begin{array}{l}\text { Web-based or smartphone with } \\
\text { wearable }(n=2)\end{array}$ & $0(0)$ & $1(6)$ & $1(7)$ \\
\hline $\begin{array}{l}\text { Web-based or smartphone with } \\
\text { wearable and monitoring device }(n=3)\end{array}$ & $1(3)$ & $2(11)$ & $0(0)$ \\
\hline \multicolumn{4}{|l|}{ Level of adherence definition } \\
\hline Category A (n=34) & $16(55)$ & $10(56)$ & $8(53)$ \\
\hline Category B $(n=23)$ & $11(38)$ & $7(39)$ & $5(33)$ \\
\hline Category C $(n=5)$ & $2(7)$ & $1(6)$ & $2(13)$ \\
\hline \multicolumn{4}{|l|}{ Number of measures ${ }^{*}$} \\
\hline $1(n=30)$ & $19(66)$ & $4(22)$ & $9(60)$ \\
\hline $2(n=17)$ & $8(28)$ & 7 (39) & $2(13)$ \\
\hline $3(n=9)$ & $1(3)$ & $3(17)$ & $3(20)$ \\
\hline 4 or more $(n=6)$ & $1(3)$ & $4(22)$ & $1(7)$ \\
\hline \multicolumn{4}{|l|}{ Measures of adherence } \\
\hline \# logins/\# sessions $(n=14)^{*}$ & $3(10)$ & $8(44)$ & $3(20)$ \\
\hline $\begin{array}{l}\text { \# modules / \# lessons completed } \\
(\mathrm{n}=30)^{*}\end{array}$ & $17(59)$ & $2(11)$ & $11(73)$ \\
\hline \# features accessed/used $(n=16)^{*}$ & $5(17)$ & $9(50)$ & $2(13)$ \\
\hline \# exercises completed $(n=9)$ & $6(21)$ & $2(11)$ & $1(7)$ \\
\hline \# pages viewed $(n=11)$ & $2(7)$ & $6(33)$ & $3(20)$ \\
\hline$\#$ days/weeks/months $(n=19)^{*}$ & $8(28)$ & $7(39)$ & $4(27)$ \\
\hline Time spent $(n=14)^{*}$ & $2(7)$ & $9(50)$ & $3(20)$ \\
\hline
\end{tabular}

${ }^{*} \mathrm{p}<.05$ 


\section{Operationalization of the adherence definition}

Out of the 62 included articles, 34 reported adherence only in terms of how often the technology was used (Category A operationalization) [7, 18-50]. In 23 studies, the intended usage was described as well (Category B operationalization) [51-73], and 5 studies reported the intended usage with a justification for this threshold (Category C operationalization) [7478]. The number of publications reporting Category C operationalizations has increased since 2015 (Figure 2).

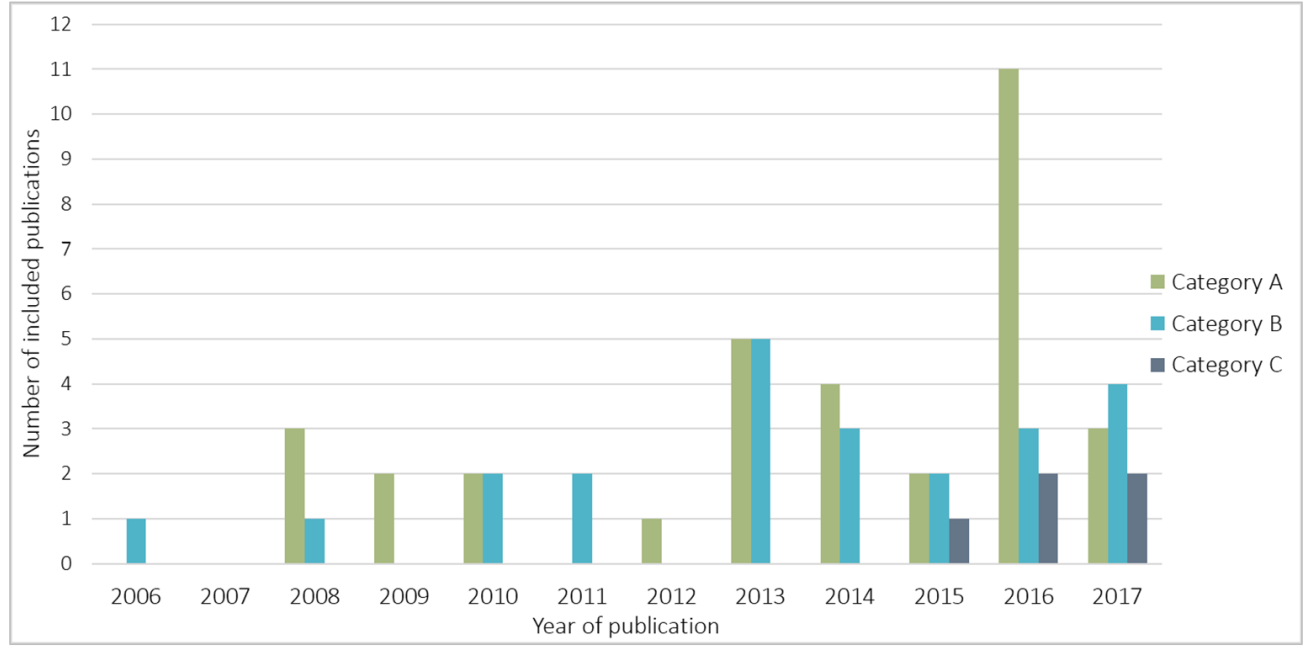

Figure 2. The number of studies reporting a Level 1, 2, and 3 operationalization per publication year.

Table 4 provides an overview of the characteristics of the studies for adherence category. Although no significant differences were found, we were still able to identify some interesting patterns. Overall, the data shows that 20/31 technologies for mental health contain a Category A operationalization, while Category C operationalizations are more equally distributed over the three healthcare fields. Furthermore, 48/56 Category $A$ and Category B operationalizations are for web-based technologies (whether or not in combination with other devices), while a third of the Category $\mathrm{C}$ operationalizations are also for smartphone technologies.

Most Category A operationalizations contain a measure for the number of modules that the users accessed or completed (19/34) and the time spent online and number of features accessed and used (both 11/34). Most Category B operationalizations contain the number of days, weeks or months that people used the technology (11/23), the number of accessed or completed modules (8/23), and the number of logins or sessions (4/23).

Category $\mathrm{C}$ definitions are mostly based on the number of accessed or completed modules (3/5 operationalizations). The number of logins and the number of days, weeks, or months 
that people used the technology were used in 2/5 operationalizations. Most operationalizations of adherence are based on a maximum of two different measures, regardless of the category (49/62). 10/13 operationalizations that feature 3 or more measures are categorized as Category A.

The included Category C operationalizations provided justification in various ways. Reinwand et al. asked all participants to complete a questionnaire to assess to what extent their lifestyle met the Dutch guidelines for healthy eating, drinking alcohol, physical activity, et cetera [74]. Recommendations for the use of corresponding elements of the technology were then made based on the outcomes of the assessment and in turn, adherence was defined as using the technology in accordance with these recommendations. In the study by Zeng et al., the technology consisted of different elements that were all evaluated as effective in other studies [75]. Users were considered adherent if they used all elements. Beatty et al. considered a user to be highly adherent when a therapeutic dose of $66 \%$ of the intervention was received [76]. This threshold for a therapeutic dose was obtained from previous studies. In the study by Mertens et al., technology use was represented in relation to medication use, and users were therefore seen as adherent when the technology was used in accordance with the recommendations for medication use [77]. Carolan et al. describe a protocol for a study to be conducted to understand the optimum adherence to the technology in relation to the outcomes [78]. 
Table 4. Characteristics of Category A, B, and C definitions

\begin{tabular}{|c|c|c|c|}
\hline & $\begin{array}{c}\text { Category A } \\
\begin{array}{c}(n=34) \\
n(\%)\end{array}\end{array}$ & $\begin{array}{c}\text { Category B } \\
\begin{array}{c}(n=23) \\
n(\%)\end{array}\end{array}$ & $\begin{array}{c}\text { Category C } \\
\qquad(n=5) \\
n(\%)\end{array}$ \\
\hline \multicolumn{4}{|l|}{ Healthcare field } \\
\hline Mental health $(n=31)$ & $20(59)$ & $9(39)$ & $2(40)$ \\
\hline Lifestyle $(n=25)$ & $13(38)$ & $10(44)$ & $2(40)$ \\
\hline Chronic care $(n=6)$ & $1(3)$ & $4(17)$ & $1(40)$ \\
\hline \multicolumn{4}{|l|}{ Device } \\
\hline Web-based $(n=51)$ & $31(91)$ & $17(74)$ & $3(60)$ \\
\hline Smartphone app $(n=6)$ & $1(3)$ & $3(13)$ & $2(40)$ \\
\hline $\begin{array}{l}\text { Web-based or smartphone with wearable } \\
(n=2)\end{array}$ & $1(3)$ & $1(4)$ & $0(0)$ \\
\hline $\begin{array}{l}\text { Web-based or smartphone with wearable and } \\
\text { monitoring device }(n=3)\end{array}$ & $1(3)$ & $2(9)$ & $0(0)$ \\
\hline \multicolumn{4}{|l|}{ Type of use } \\
\hline Structured $(n=29)$ & $16(47)$ & $11(48)$ & $2(40)$ \\
\hline Unstructured $(n=18)$ & $10(29)$ & $7(30)$ & $1(20)$ \\
\hline Hybrid $(n=15)$ & $8(24)$ & $5(22)$ & $2(40)$ \\
\hline \multicolumn{4}{|l|}{ Measures of adherence } \\
\hline \# logins/\# sessions $(n=14)$ & $8(24)$ & $4(17)$ & $2(40)$ \\
\hline \# modules / \# lessons completed $(n=30)$ & $19(56)$ & $8(35)$ & $3(60)$ \\
\hline \# features accessed/used $(n=16)$ & $11(32)$ & $4(17)$ & $1(20)$ \\
\hline \# exercises completed $(n=9)$ & $6(18)$ & $3(13)$ & $0(0)$ \\
\hline \# pages viewed $(n=11)$ & $9(27)$ & $1(4)$ & $1(20)$ \\
\hline \# days/weeks/months $(n=19)^{*}$ & $6(18)$ & $11(48)$ & $2(40)$ \\
\hline Time spent $(n=14)$ & $11(32)$ & $2(9)$ & $1(17)$ \\
\hline \multicolumn{4}{|l|}{ Number of measures } \\
\hline $1(n=32)$ & $13(38)$ & $16(70)$ & $3(60)$ \\
\hline $2(n=17)$ & $11(32)$ & $5(22)$ & $1(20)$ \\
\hline $3(n=7)$ & $6(18)$ & $1(4)$ & $0(0)$ \\
\hline 4 or more $(n=6)$ & $4(12)$ & $1(4)$ & $1(20)$ \\
\hline
\end{tabular}

* $p<.05$ 


\section{Discussion}

In this systematic review, we have sought to gain insight into how the concept of adherence has been used in previous eHealth evaluations. In line with the definitions for adherence and intended use maintained by the WHO [10] and Kelders [1], we reviewed not only how usage was measured, but also if and how intended use of eHealth technologies was operationalized and justified using theory, evidence or rationale.

\section{Principal Results}

We included 62 studies in this review, all published after 2005. The majority of the technologies described in these studies were structured or hybrid web-based interventions targeting mental health (mostly CBT interventions) or unstructured technologies for lifestyle support.

We observed a growing number of studies that studied adherence to eHealth technologies since Eysenbach's plea for reporting the levels of non-usage attrition with eHealth technology in 2005 [9]. Although the 'prescribed recommendations' or the intended use of a technology form an important element of the definition of adherence [10], and although there is evidence that users do not always have to complete an intervention in order to experience effects [6, 11, 13-15], half of all operationalizations are based on the assumption of 'the more use, the better' and do not include a threshold for intended use. Sometimes we do not know (yet) what the intended use of a technology is, or defining the intended use is not necessary to answer the research question(s) of the study. Then, a Category A operationalization suffices for answering the research questions. However, a Category A operationalization only refers to the actual usage of a technology without comparing it to its intended usage. According to the definitions used, they should therefore not be referred to as adherence.

When the intended use for the technology was reported, only a minority of all included studies featured justified Category $\mathrm{C}$ operationalizations, making the comparison of adherence across different eHealth technologies more complicated. However, we were still able to observe a small increase in Category C operationalizations since 2015.

Remarkably, the proportion of evaluations of mental health technologies reporting a justified (Category C) operationalization of intended use is lagging behind compared to evaluations of lifestyle and chronic care technologies. This is unexpected, since the majority of mental health technologies is based on (principles of) CBT, which is the most studied treatment for depression and has proven effective in many studies [79]. However, a meta-analysis of Van Ballegooijen et al. revealed that participants complete approximately $84 \%$ of their CBT program in both offline and online treatment. Although a longer treatment duration is associated with better effects [80], this still implies that users do not necessarily need to 
finish the complete program in order to experience reduction of their complaints, and that there should be a threshold for intended use. As such, it seems that this knowledge could be used to define and substantiate the intended use of a CBT technology, but in the studies included in this review this notion has not been put into practice yet.

We were also able to observe some interesting patterns in the composition of measures for adherence and intended use. When the operationalization of adherence consists of a combination of four or more measures, it is most likely a Category A operationalization, while most Category $C$ operationalizations consist of one or two measures. This implies that for many Category A operationalizations, a scattershot approach was used when it comes to measuring adherence. In contrast, the results indicated that justifications of intended use are often based on the goal of the technology and/or the assumed working mechanisms, leading to more focused operationalizations that do not require additional measurements to evaluate adherence to the technology. In other words, more measures are not necessarily the key to knowledge if they are not sufficiently specific.

No significant differences could be found between the kinds of measures that are used for all three levels of operationalizations. Category A operationalizations most often contain the number of modules that a user started or completed, the number of features accessed or used, and the time spent online. This seems obvious, as this level of operationalization is mostly used for structured or hybrid mental health interventions consisting of different modules that users have to follow. Category C or justified operationalizations are more often defined by the number of days, weeks or months that the technology is used by people. This can be explained by way of the finding that Category $C$ operationalizations are used for a large proportion of unstructured and hybrid technologies. As people are able use the features of these technologies more or less at their own convenience, the development of use over time would probably provide more information regarding adherence than use of the technology's content at fixed points in time only.

\section{Implications and recommendations}

An important reason for the lack of justifications for the intended use of eHealth technologies might be that there is a lack of knowledge regarding the working mechanisms of technologybased applications [8]. However, the included Category $C$ operationalizations did show that knowledge of the working mechanisms of the technology is not a prerequisite for defining the intended use. After all, the intended use or the 'therapeutic dose' can be justified just as well using existing guidelines for healthy living and medication use [74, 77] or using previous research regarding other technologies $[75,76]$. Moreover, the intended use has also been operationalized by linking the (positive) outcomes of individual users to their usage patterns to find the most effective patterns [78]. 
The fact that we did not find a justification of intended use based on existing models for behavior change was unexpected. For instance, Kaushal \& Rhodes found that exercising for at least four times per week for six weeks is a minimal requirement for establishing exercise habits [81]. These kind of findings can also be used for determining intended use; for example, a user of a technology to improve physical activity is adherent when using (specific elements of) the technology at least four times a week and six weeks in a row.

Another example comes from Kelders et al., who found that a group of users dropped out from an intervention for reducing depressive complaints after a lesson that focused on applying newly acquired skills in practice, as doing so can be confrontational [56]. However, following this lesson can also be seen as an important precondition for gaining effects from using the intervention. The intended use of this intervention could thus be operationalized as following the intervention until that critical lesson is completed at the very least. An important aspect of operationalizing the intended use of a technology is therefore to keep the goal of the technology and the desired outcomes in mind. What use is necessary at minimum to reach that goal (e.g. to experience certain effects or establish new skills and habits), and how can we translate this into measures for adherence?

Although it has previously been suggested that a combination of a range of different variables for technology usage provides a more meaningful measure of adherence [6], the results of this review show that a limited but deliberate set of only one or two different measures in accordance with the goal of the technology can also be used for operationalizing intended use. At the moment, eHealth evaluations often fail to demonstrate the dose-response relationship (the usage that is minimally needed to experience certain effects) or simply define it as 'the more use, the better'. However, the results of this review indicate that Category $A$ and $B$ operationalizations of adherence often do not take the characteristics of the technology (e.g., goal, persuasiveness, user-friendliness) into account. It is thus very possible that dose-response relationships might become more apparent when the measures used to operationalize adherence match the goal of the technology [82, 83].

All of the measures for adherence in this review are based on data regarding technology usage. However, the results of a recent literature review of theoretical perspectives on adherence showed that adherence is a multidimensional concept, influenced by a range of technological, environmental and individual factors altogether that cannot be evaluated by technology usage alone $[8,84]$. Therefore, additional measures are needed to determine whether and why users are or have been adherent to technology. For example, a mixed methods approach that combines usage data with questionnaires, health measurements, and/or interviews could provide important knowledge regarding why people do or do not use the technology, how people learn from using the technology, the minimal use that is needed for users to experience certain effects or to reach certain goals, and how the skills 
acquired while using the technology are then applied in daily life. These outcomes could then in turn be used for determining the intended use of the technology and translating that concept into concrete measures for evaluating adherence.

In their review, Donkin et al. state that it is difficult to compare adherence to different technologies when the measures that are used across the different trials vary [6]. However, this statement is based on Category A operationalizations of adherence where more use is better. When using Category B or (preferably) Category $C$ operationalizations, the actual usage of each individual can be compared to the technology's intended usage. In turn, the percentage of people who adhered to the intervention can be calculated, making adherence a more objective and standardized concept [1]. This approach simplifies the comparison of adherence across technologies with the same goal (e.g., improving physical activity) but different technology characteristics (e.g., features of the technology, persuasiveness, userfriendliness) and a different operationalization of intended use. At the same time, this approach also simplifies the comparison of adherence of different users of a specific technology. Ideally, when individuals have different goals for using a technology, they should also have an individual intended usage, which could be used to get more fine-grained, personalized measures of adherence. This will be of added value for both developers and researchers, as this approach will provide better insight into how adherence to the various elements of different technologies contributed to the outcomes that are found, and for whom.

\section{Limitations}

As the goal of this review was to gain insight into how the concept of adherence has been used in previous eHealth evaluations, we only included studies that used adherence as an outcome measure or studies that explicitly stated how other outcome measures are used as a proxy for adherence. As such, we may have missed relevant studies that formulated the intended use of a technology, but used other related terms for adherence (e.g., non-usage attrition, engagement, drop-out or (non-)usage). In future research, added value might be obtained by reviewing these studies as well, in order to find directions on how to operationalize the intended use for different eHealth technologies.

Second, we defined the categories of adherence operationalizations for every study, instead of every type of technology. After all, adherence and intended use can be defined in many different ways and it is very possible for different operationalizations to be used for different studies regarding the same technology. Even so, we feel that we have included a large body of studies in this review, providing valuable insight into the concept of adherence and intended use. 


\section{Conclusions}

Previous research has shown that users do not always have to experience all of the elements of a technology and that effective usage patterns might differ across users. However, the results of this review show that the operationalization of intended use is mostly based on the assumption of 'the more use, the better' and that when a threshold of intended use is provided, justification is often missing. Therefore, it can be concluded that adherence to eHealth technology is an underdeveloped and often improperly used concept in the existing body of literature.

When the intended use of a technology was defined, the goal of the technology and/or the assumed working mechanisms often formed the starting point for selecting valid measures (e.g., number of logins, number of completed modules). A justified threshold for intended use in accordance with the goal of the technology provides information for a concise evaluation of adherence and the working mechanisms of a technology. Subsequently, justified operationalization (comprised of multidimensional measures or not) can be used to standardize adherence to different eHealth technologies, making it easier to compare the adherence rates of different technologies. 


\section{References}

1. Kelders SM, Kok RN, Ossebaard HC, Van Gemert-Pijnen JEWC. Persuasive system design does matter: A systematic review of adherence to web-based interventions. Journal of Medical Internet Research. 2012;14(6):p16-p39.

2. Elbert NJ, van Os-Medendorp H, van Renselaar W, Ekeland AG, Hakkaart-van Roijen L, Raat H, et al. Effectiveness and Cost-Effectiveness of eHealth Interventions in Somatic Diseases: A Systematic Review of Systematic Reviews and Meta-Analyses. J Med Internet Res. 2014;16(4):e110.

3. Beishuizen CR, Stephan BC, van Gool WA, Brayne C, Peters RJ, Andrieu S, et al. Web-Based Interventions Targeting Cardiovascular Risk Factors in Middle-Aged and Older People: A Systematic Review and Meta-Analysis. J Med Internet Res. 2016;18(3):e55. .

4. Hadjiconstantinou M, Byrne J, Bodicoat DH, Robertson N, Eborall H, Khunti K, et al. Do Web-Based Interventions Improve Well-Being in Type 2 Diabetes? A Systematic Review and Meta-Analysis. J Med Internet Res. 2016;18(10):e270.

5. Kooij L, Groen WG, van Harten WH. The Effectiveness of Information Technology-Supported Shared Care for Patients With Chronic Disease: A Systematic Review. J Med Internet Res. 2017;19(6):e221.

6. Donkin L, Christensen H, Naismith SL, Neal B, Hickie IB, Glozier N. A systematic review of the impact of adherence on the effectiveness of e-therapies. Journal of Medical Internet Research. 2011;13(3):81-92

7. Manwaring JL, Bryson SW, Goldschmidt AB, Winzelberg AJ, Luce KH, Cunning D, et al. Do adherence variables predict outcome in an online program for the prevention of eating disorders? Journal of Consulting and Clinical Psychology. 2008;76(2):341-6.

8. Michie S, Yardley L, West R, Patrick K, Greaves F. Developing and Evaluating Digital Interventions to Promote Behavior Change in Health and Health Care: Recommendations Resulting From an International Workshop. J Med Internet Res. 2017;19(6):e232.

9. Eysenbach G. The law of attrition. Journal of Medical Internet Research. 2005;7(1).

10. Sabaté E. Adherence to long-term therapies: evidence for action: World Health Organization; 2003. ISBN: 9241545992.

11. Christensen H, Griffiths KM, Farrer L. Adherence in Internet intervention for anxiety and depression: Systematic review. Journal of Medical Internet Research. 2009;11(2):1-16.

12. Smith W, Ploderer B, Wadley G, Webber S, Borland R. Trajectories of Engagement and Disengagement with a Story-Based Smoking Cessation App. Proceedings of the $2017 \mathrm{CHI}$ Conference on Human Factors in Computing Systems; Denver, Colorado, USA. 3026054: ACM; 2017. p. 3045-56.

13. Hekler EB, Klasnja P, Riley WT, Buman MP, Huberty J, Rivera DE, et al. Agile science: creating useful products for behavior change in the real world. Translational Behavioral Medicine. 2016;6(2):31728.

14. Moller AC, Merchant G, Conroy DE, West R, Hekler E, Kugler KC, et al. Applying and advancing behavior change theories and techniques in the context of a digital health revolution: proposals for more effectively realizing untapped potential. Journal of Behavioral Medicine. 2017;40(1):85-98.

15. Christensen $\mathrm{H}$, Mackinnon A. The law of attrition revisited. Journal of Medical Internet Research. 2006;8(3):70-2. 
16. van Ballegooijen W, Cuijpers P, van Straten A, Karyotaki E, Andersson G, Smit JH, et al. Adherence to Internet-Based and Face-to-Face Cognitive Behavioural Therapy for Depression: A Meta-Analysis. PLOS ONE. 2014;9(7):e100674.

17. Beatty L, Binnion C. A Systematic Review of Predictors of, and Reasons for, Adherence to Online Psychological Interventions. International Journal of Behavioral Medicine. 2016:1-19.

18. Alaoui SE, Ljótsson B, Hedman E, Kaldo V, Andersson E, Rück C, et al. Predictors of symptomatic change and adherence in Internet-based cognitive behaviour therapy for social anxiety disorder in routine psychiatric care. PLOS ONE. 2015;10(4).

19. Alfonsson S, Olsson E, Hursti T. Motivation and treatment credibility predicts dropout, treatment adherence, and clinical outcomes in an internet-based cognitive behavioral relaxation program: A randomized controlled trial. Journal of Medical Internet Research. 2016;18(3).

20. Alfonsson S, Olsson E, Linderman S, Winnerhed S, Hursti T. Is online treatment adherence affected by presentation and therapist support? A randomized controlled trial. Computers in Human Behavior. 2016;60:550-8.

21. Batterham PJ, Neil AL, Bennett K, Griffiths KM, Christensen H. Predictors of adherence among community users of a cognitive behavior therapy website. Patient Preference and Adherence. 2008;2:97-105.

22. Beintner I, Jacobi C, Taylor CB. Participant adherence to the Internet-based prevention program StudentBodies ${ }^{\mathrm{TM}}$ for eating disorders - A review. Internet Interventions. 2014 3//;1(1):26-32.

23. Christensen H, Griffiths K, Groves C, Korten A. Free range users and one hit wonders: Community users of an Internet-based cognitive behaviour therapy program. Australian and New Zealand Journal of Psychiatry. 2006;40(1):59-62.

24. Graham AL, Cha S, Cobb NK, Fang Y, Niaura RS, Mushro A. Impact of seasonality on recruitment, retention, adherence, and outcomes in a web-based smoking cessation intervention: Randomized controlled trial. Journal of Medical Internet Research. 2013;15(11).

25. Graham AL, Cha S, Papandonatos GD, Cobb NK, Mushro A, Fang Y, et al. Improving adherence to web-based cessation programs: A randomized controlled trial study protocol. Trials. 2013:48.

26. Graham AL, Jacobs MA, Cohn AM, Cha S, Abroms LC, Papandonatos GD, et al. Optimising text messaging to improve adherence to web-based smoking cessation treatment: A randomised control trial protocol. BMJ Open. 2016;6(3).

27. Ho FY-Y, Chung K-F, Yeung W-F, Ng TH-Y, Cheng SK-W. Weekly brief phone support in self-help cognitive behavioral therapy for insomnia disorder: Relevance to adherence and efficacy. Behaviour Research and Therapy. 2014;63:147-56

28. Johansson O, Michel T, Andersson G, Paxling B. Experiences of non-adherence to Internet-delivered cognitive behavior therapy: A qualitative study. Internet Interventions. 2015;2(2):137-42.

29. Mañanes G, Vallejo MA. Usage and effectiveness of a fully automated, open-access, Spanish Webbased smoking cessation program: Randomized controlled trial. Journal of Medical Internet Research. 2014;16(4):1-18.

30. Lillevoll KR, Vangberg HCB, Griffiths KM, Waterloo K, Eisemann MR. Uptake and adherence of a selfdirected internet-based mental health intervention with tailored e-mail reminders in senior high schools in Norway. BMC Psychiatry. 2014;14.

31. McCabe MP, Price E. Attrition from an internet-based psychological intervention for erectile dysfunction: Who is likely to drop out? Journal of Sex and Marital Therapy. 2009;35(5):391-401. 
32. Mohr DC, Duffecy J, Ho J, Kwasny M, Cai X, Burns MN, et al. A Randomized Controlled Trial Evaluating a Manualized TeleCoaching Protocol for Improving Adherence to a Web-Based Intervention for the Treatment of Depression. PLOS ONE. 2013;8(8).

33. Murray E, White IR, Varagunam M, Godfrey C, Khadjesari Z, McCambridge J. Attrition revisited: Adherence and retention in a web-based alcohol trial. Journal of Medical Internet Research. 2013;15(8).

34. Neil AL, Batterham P, Christensen H, Bennett K, Griffiths KM. Predictors of adherence by adolescents to a cognitive behavior therapy website in school and community-based settings. Journal of Medical Internet Research. 2009;11(1):42-9.

35. Neve MJ, Collins CE, Morgan PJ. Dropout, nonusage attrition, and pretreatment predictors of non usage attrition in a commercial web-based weight loss program. Journal of Medical Internet Research. 2010;12(4):81-96.

36. Price M, Davidson TM, Andrews JO, Ruggiero KJ. Access, use and completion of a brief disaster mental health intervention among Hispanics, African-Americans and Whites affected by Hurricane Ike. Journal of Telemedicine and Telecare. 2013;19(2):70-4.

37. Price M, Gros DF, McCauley JL, Gros KS, Ruggiero KJ. Nonuse and dropout attrition for a web-based mental health intervention delivered in a post-disaster context. Psychiatry: Interpersonal and Biological Processes. 2012 Fal 2012;75(3):267-84.

38. Wanner M, Martin-Diener E, Bauer G, Braun-Fahrlander C, Martin BW. Comparison of trial participants and open access users of a Web-based physical activity intervention regarding adherence, attrition, and repeated participation. Journal of Medical Internet Research. 2010;12(1).

39. Ware LJ, Hurling R, Bataveljic O, Fairley BW, Hurst TL, Murray P, et al. Rates and determinants of uptake and use of an internet physical activity and weight management program in office and manufacturing work sites in England: Cohort study. Journal of Medical Internet Research. 2008;10(4).

40. Blake H, Quirk H, Leighton P, Randell T, Greening J, Guo B, et al. Feasibility of an online intervention (STAK-D) to promote physical activity in children with type 1 diabetes: Protocol for a randomised controlled trial. Trials. 2016;17(1).

41. Graham AL, Papandonatos GD, Cha S, Erar B, Amato MS, Cobb NK, et al. Improving Adherence to Smoking Cessation Treatment: Intervention Effects in a Web-Based Randomized Trial. Nicotine \& Tobacco Research. 2017 Mar;19(3):324-32.

42. Grossert A, Urech C, Alder J, Gaab J, Berger T, Hess V. Web-based stress management for newly diagnosed cancer patients (STREAM-1): A randomized, wait-list controlled intervention study. BMC Cancer. 2016;16(1).

43. Hayman M, Reaburn P, Browne M, Vandelanotte C, Alley S, Short CE. Feasibility, acceptability and efficacy of a web-based computer-tailored physical activity intervention for pregnant women - the Fit4Two randomised controlled trial. BMC Pregnancy Childbirth. 2017 Mar;17:10.

44. Jander A, Crutzen R, Mercken L, Candel M, de Vries H. Effects of a Web-based computer-tailored game to reduce binge drinking among Dutch adolescents: A cluster randomized controlled trial. Journal of Medical Internet Research. 2016;18(2):1-17.

45. Kooistra LC, Ruwaard J, Wiersma JE, van Oppen P, van der Vaart R, van Gemert-Pijnen JEWC, et al. Development and initial evaluation of blended cognitive behavioural treatment for major depression in routine specialized mental health care. Internet Interventions. 2016;4:61-71. 
46. Noone C, Hogan MJ. A protocol for a randomised activecontrolled trial to evaluate the effects of an online mindfulness intervention on executive control, critical thinking and key thinking dispositions in a university student sample. BMC Psychology. 2016;4(1).

47. Nordin CA, Michaelson P, Gard G, Eriksson MK. Effects of the Web Behavior Change Program for Activity and multimodal pain rehabilitation: Randomized controlled trial. Journal of Medical Internet Research. 2016;18(10):24-41.

48. Schulz A, Stolz T, Vincent A, Krieger T, Andersson G, Berger T. A sorrow shared is a sorrow halved? A three-arm randomized controlled trial comparing internet-based clinician-guided individual versus group treatment for social anxiety disorder. Behaviour Research and Therapy. 2016 9//;84:14-26.

49. Tu AW, Watts AW, Chanoine JP, Panagiotopoulos C, Geller J, Brant R, et al. Does parental and adolescent participation in an e-health lifestyle modification intervention improves weight outcomes? BMC Public Health. 2017;17(1).

50. Wang Z, Wang J, Maercker A. Program use and outcome change in a Web-based trauma intervention: Individual and social factors. Journal of Medical Internet Research. 2016;18(9):4-17.

51. An LC, Perry CL, Lein EB, Klatt C, Farley DM, Bliss RL, et al. Strategies for increasing adherence to an online smoking cessation intervention for college students. Nicotine \& Tobacco Research. 2006;8(Suppl 1):S7-S12

52. Carter MC, Burley VJ, Nykjaer C, Cade JE. Adherence to a smartphone application for weight loss compared to website and paper diary: Pilot randomized controlled trial. Journal of Medical Internet Research. 2013;15(4):56-72.

53. Cruz VT, Pais J, Alves I, Ruano L, Mateus C, Barreto R, et al. Web-Based Cognitive Training: Patient Adherence and Intensity of Treatment in an Outpatient Memory Clinic. Journal of Medical Internet Research. 2014 May;16(5):130-40.

54. Farrer LM, Griffiths KM, Christensen H, Mackinnon AJ, Batterham PJ. Predictors of adherence and outcome in internet-based cognitive behavior therapy delivered in a telephone counseling setting. Cognitive Therapy and Research. 2014;38(3):358-67.

55. Joseph RP, Dutton GR, Cherrington A, Fontaine K, Baskin M, Casazza K, et al. Feasibility, acceptability, and characteristics associated with adherence and completion of a culturally relevant internet-enhanced physical activity pilot intervention for overweight and obese young adult African American women enrolled in college. BMC Research Notes. 2015.

56. Kelders SM, Bohlmeijer ET, Van Gemert-Pijnen J. Participants, Usage, and Use Patterns of a WebBased Intervention for the Prevention of Depression Within a Randomized Controlled Trial. Journal of Medical Internet Research. 2013 Aug;15(8).

57. Mâsse LC, Watts AW, Barr SI, Tu AW, Panagiotopoulos C, Geller J, et al. Individual and household predictors of adolescents' adherence to a Web-based intervention. Annals of Behavioral Medicine. 2015;49(3):371-83.

58. Mattila E, Orsama AL, Ahtinen A, Hopsu L, Leino T, Korhonen I. Personal health technologies in employee health promotion: Usage activity, usefulness, and health-related outcomes in a 1-year randomized controlled trial. Journal of Medical Internet Research. 2013;15(7).

59. Nicholas J, Proudfoot J, Parker G, Gillis I, Burckhardt R, Manicavasagar V, et al. The Ins and Outs of an Online Bipolar Education Program: A Study of Program Attrition. Journal of Medical Internet Research. 2010;12(5). 
60. Postel MG, Haan HAd, Huurne EDt, van der Palen J, Becker ES, de Jong CAJ. Attrition in web-based treatment for problem drinkers. Journal of Medical Internet Research. 2011;13(4):120-33.

61. Richardson CR, Buis LR, Janney AW, Goodrich DE, Sen A, Hess ML, et al. An online community improves adherence in an Internet-mediated walking program. Part 1: Results of a randomized controlled trial. Journal of Medical Internet Research. 2010;12(4):138-53.

62. Rodgers RF, Franko DL, Shiyko M, Intille S, Wilson K, O'Carroll D, et al. Exploring healthy eating among ethnic minority students using mobile technology: Feasibility and adherence. Health Informatics Journal. 2016;22(3):440-50.

63. Titov N, Dear BF, Johnston L, Lorian C, Zou J, Wootton B, et al. Improving Adherence and Clinical Outcomes in self- guided internet treatment for anxiety and depression: randomised controlled trial. PLoS ONE. 2013;8(7).

64. Van den Berg SW, Peters EJ, Kraaijeveld JF, Gielissen MFM, Prins JB. Usage of a generic web-based self-management intervention for breast cancer survivors: Substudy analysis of the BREATH trial. Journal of Medical Internet Research. 2013;15(8):30-40.

65. Van der Zanden R, Curie K, Van Londen M, Kramer J, Steen G, Cuijpers P. Web-based depression treatment: Associations of clients' word use with adherence and outcome. Journal of Affective Disorders. 2014;160:10-3.

66. Wangberg SC, Bergmo TS, Johnsen JAK. Adherence in Internet-based interventions. Patient Preference and Adherence. 2008;2:57-65.

67. Alley S, Jennings C, Plotnikoff RC, Vandelanotte C. Web-based video-coaching to assist an automated computer-tailored physical activity intervention for inactive adults: A randomized controlled trial. Journal of Medical Internet Research. 2016;18(8).

68. Carlsen K, Jakobsen C, Houen G, Kallemose T, Paerregaard A, Riis LB, et al. Self-managed eHealth Disease Monitoring in Children and Adolescents with Inflammatory Bowel Disease: A Randomized Controlled Trial. Inflammatory Bowel Diseases. 2017;23(3):357-65.

69. Fernandes-Taylor S, Gunter RL, Bennett KM, Awoyinka L, Rahman S, Greenberg CC, et al. Feasibility of Implementing a Patient-Centered Postoperative Wound Monitoring Program Using Smartphone Images: A Pilot Protocol. JMIR RES Protoc. 2017 Feb;6(2):12.

70. Rutledge T, Skoyen JA, Wiese JA, Ober KM, Woods GN. A comparison of MOVE! versus TeleMOVE programs for weight loss in Veterans with obesity. Obesity Research and Clinical Practice. 2016.

71. Schuster R, Leitner I, Carlbring P, Laireiter A-R. Exploring blended group interventions for depression: Randomised controlled feasibility study of a blended computer- and multimediasupported psychoeducational group intervention for adults with depressive symptoms. Internet Interventions. 2017;8:63-71.

72. Zarski AC, Lehr D, Berking M, Riper H, Cuijpers P, Ebert DD. Adherence to Internet-Based MobileSupported Stress Management: A Pooled Analysis of Individual Participant Data From Three Randomized Controlled Trials. Journal of Medical Internet Research. 2016 Jun;18(6):15.

73. Nijland N, van Gemert-Pijnen JEWC, Kelders SM, Brandenburg BJ, Seydel ER. Factors influencing the use of a web-based application for supporting the self-care of patients with type 2 diabetes: A longitudinal study. Journal of Medical Internet Research. 2011;13(3):118-33.

74. Reinwand DA, Schulz DN, Crutzen R, Kremers SPJ, de Vries H. Who follows eHealth interventions as recommended? A study of participants' personal characteristics from the experimental arm of a randomized controlled trial. Journal of Medical Internet Research. 2015;17(5). 
75. Zeng EY, Heffner JL, Copeland WK, Mull KE, Bricker JB. Get with the program: Adherence to a smartphone app for smoking cessation. Addictive Behaviors. 2016;63:120-4.

76. Beatty L, Kemp E, Binnion C, Turner J, Milne D, Butow P, et al. Uptake and adherence to an online intervention for cancer-related distress: older age is not a barrier to adherence but may be a barrier to uptake. Supportive Care in Cancer. 2017;25(6):1905-14.

77. Mertens A, Becker S, Theis S, Rasche $P$, Wille M, Brohl C, et al. Mobile Technology Improves Therapy-Adherence Rates in Elderly Patients Undergoing Rehabilitation-A Crossover Design Study. In: Duffy VG, Lightner N, editors. Advances in Human Factors and Ergonomics in Healthcare. Cham: Springer Int Publishing Ag; 2017. p. 295-308.

78. Carolan S, Harris PR, Greenwood K, Cavanagh K. Increasing engagement with, and effectiveness of, an online CBT-based stress management intervention for employees through the use of an online facilitated bulletin board: study protocol for a pilot randomised controlled trial. Trials. 2016 Dec;17:10.

79. Cuijpers P, Berking M, Andersson G, Quigley L, Kleiboer A, Dobson KS. A Meta-Analysis of CognitiveBehavioural Therapy for Adult Depression, Alone and in Comparison with other Treatments. The Canadian Journal of Psychiatry. 2013;58(7):376-85.

80. Zachariae R, Lyby MS, Ritterband LM, O'Toole MS. Efficacy of internet-delivered cognitivebehavioral therapy for insomnia - A systematic review and meta-analysis of randomized controlled trials. Sleep Medicine Reviews. 2016;30:1-10.

81. Kaushal N, Rhodes RE. Exercise habit formation in new gym members: a longitudinal study. Journal of Behavioral Medicine. 2015;38(4):652-63.

82. Sieverink F, Kelders SM, Poel M, Van Gemert-Pijnen JEWC. Opening the Black Box of Electronic Health: Collecting, Analyzing, and Interpreting Log Data. JMIR RES Protoc. 2017.

83. Van Gemert-Pijnen JE, Kelders SM, BohImeijer ET. Understanding the Usage of Content in a Mental Health Intervention for Depression: An Analysis of Log Data. J Med Internet Res. 2014;16(1):e27.

84. Ryan C, Bergin M, Wells JS. Theoretical Perspectives of Adherence to Web-Based Interventions: a Scoping Review. International Journal of Behavioral Medicine. 2017 July 20. 


\section{Appendix 1. Keywords literature search}

\begin{tabular}{|l|l|l|l|}
\hline Technology & Intervention & Adherence & Health \\
\hline "web*page" & Intervention* & *Adheren* & Health* \\
\hline "web*application" & Treatment* & & Behavio* \\
\hline website & Program* & & "self*help" \\
\hline "Internet*delivered" & Therap* & "self*control" \\
\hline "web*based" & Coach* & "self*management" \\
\hline "internet*based" & Platform* & "self*are" \\
\hline "internet*mediated" & App* & \\
\hline "internet*supported" & & & \\
\hline online* & & & \\
\hline "medical informatics" & & & \\
\hline "information tech*" & & & \\
\hline "e*health" & & & \\
\hline "e*therap*" & & & \\
\hline telemedic* & & & \\
\hline Telecare & & & \\
\hline Telehealth & & & \\
\hline "e*mental health" & & & \\
\hline "emental health" & & & \\
\hline Wearable* & & & \\
\hline M*health & & & \\
\hline "Mobile tech*" & & & \\
\hline "Smart*watch*" & & & \\
\hline
\end{tabular}


Appendix 2. Included studies and technologies, levels of adherence operationalization, and measures 


\begin{tabular}{|c|c|c|c|c|c|c|c|c|c|c|c|c|c|c|c|}
\hline 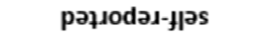 & & $x$ & & & & & & & & & & & & & \\
\hline żodoup-^pm,s & & & $\times$ & & & & $\times$ & & & & & & & & \\
\hline ұuads әu!! & & & & & & $x$ & & & & & & & & & \\
\hline 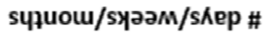 & & & & & & $\times$ & & & $\times$ & & $x$ & & & & \\
\hline səรిed \# & & & & & & $\times$ & $x$ & $x$ & & & & & & & \\
\hline səsı̣วนวха \# & $x$ & $\times$ & & & & & & & & & & & & & \\
\hline sұนәшәр \# & $\times$ & $\times$ & & $\times$ & & & & $x$ & & & & & & & \\
\hline suossə|/sə|npou \# & & & $x$ & & $x$ & $\times$ & & & & & & $x$ & & $x$ & $\times$ \\
\hline suo!̣sas/su!̨o| \# & & & & & & $\times$ & & & & $x$ & & & $x$ & & \\
\hline ¿рәұе!̨uеłsqns & $\stackrel{\circ}{z}$ & ż & ? & $\stackrel{\circ}{2}$ & $\stackrel{0}{z}$ & $\stackrel{y}{=}$ & $\stackrel{\circ}{z}$ & ? & $\stackrel{0}{z}$ & 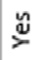 & 2 & $\stackrel{0}{z}$ & $\stackrel{\circ}{z}$ & 울 & $\stackrel{\circ}{z}$ \\
\hline ¿ə2ిesn pәрuәұи| & $\stackrel{2}{2}$ & 2 & $\check{y}$ & $\stackrel{y}{=}$ & 2 & $\stackrel{y}{\rightleftharpoons}$ & 2 & $\stackrel{2}{2}$ & y & 气 & 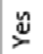 & $\stackrel{9}{z}$ & 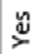 & $2^{\circ}$ & $\stackrel{2}{\check{y}}$ \\
\hline 总 & 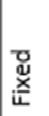 & $\begin{array}{l}\text { D. } \\
\text { 这 }\end{array}$ & 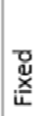 & ఖัّ & $\begin{array}{l}\text { 은 } \\
\text { 主 }\end{array}$ & $\begin{array}{l}\text { 름 } \\
\text { 롯 }\end{array}$ & 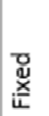 & 巡 & 离 & $\begin{array}{l}\text { 은 } \\
\text { 竞 }\end{array}$ & 巡 & $\begin{array}{l}\text { 은 } \\
\text { 主 }\end{array}$ & 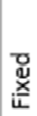 & 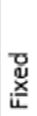 & $\begin{array}{l}\text { 은 } \\
\text { 齐 }\end{array}$ \\
\hline هั & 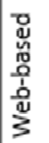 & 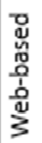 & 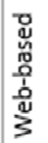 & 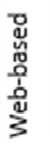 & 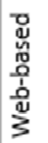 & 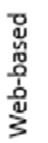 & 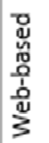 & 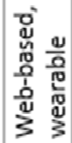 & 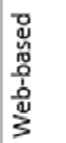 & 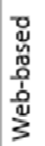 & 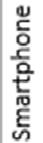 & 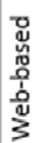 & 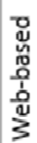 & 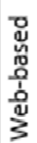 & 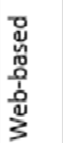 \\
\hline 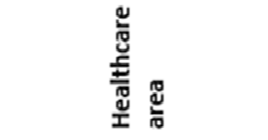 & 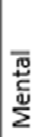 & 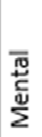 & 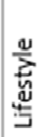 & 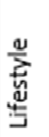 & $\begin{array}{l}\text { 苞 } \\
\text { 㟧 }\end{array}$ & 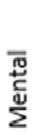 & 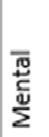 & 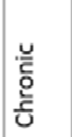 & 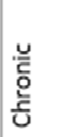 & 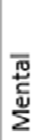 & 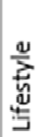 & 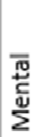 & 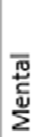 & $\begin{array}{l}\text { 焉 } \\
\text { 㟧 }\end{array}$ & 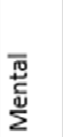 \\
\hline 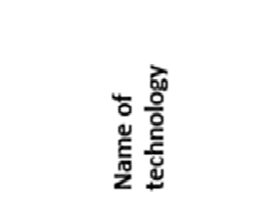 & $\stackrel{\pi}{=}$ & $\stackrel{n}{2}$ & 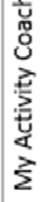 & 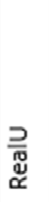 & $\begin{array}{l}\frac{5}{J} \\
\frac{1}{0} \\
\frac{0}{2}\end{array}$ & 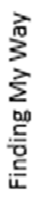 & 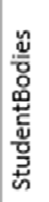 & 旁 & 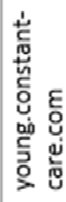 & 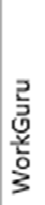 & 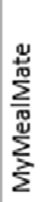 & 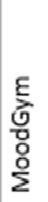 & 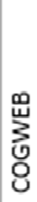 & $\stackrel{m}{=}$ & 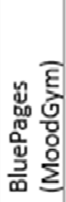 \\
\hline ֻ & $\stackrel{\bullet}{\circ}$ & $\stackrel{\bullet}{\circ}$ & 올 & ర్లి & రัలి & 공 & ఫ্ন & 올 & 공 & $\stackrel{\bullet}{\circ}$ & ํㅗㅁ & นัญ & ఫ্ন & ํํำ & ন্ন \\
\hline 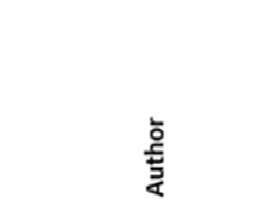 & 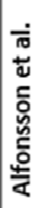 & 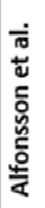 & 离 & $\begin{array}{l}\text { 荡 } \\
\frac{ \pm}{4}\end{array}$ & 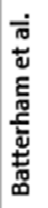 & 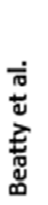 & 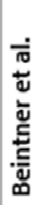 & 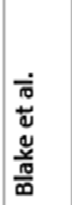 & 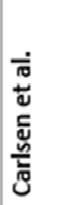 & $\begin{array}{l}\frac{10}{10} \\
\frac{ \pm}{0} \\
\frac{10}{0} \\
\frac{0}{8}\end{array}$ & 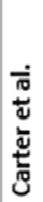 & 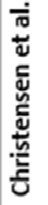 & 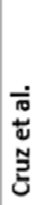 & 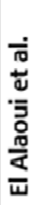 & 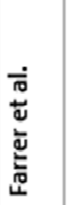 \\
\hline
\end{tabular}




\begin{tabular}{|c|c|c|c|c|c|c|c|c|c|c|c|c|c|c|c|c|c|c|}
\hline & & & & & & & $\times$ & & & & & & & & & & & \\
\hline & $\times$ & $\times$ & $\times$ & $\times$ & $\times$ & & $\times$ & & & $\times$ & & & & & & & & \\
\hline$\times$ & & $\times$ & & & & & & & & & & & & & $\times$ & $\times$ & $\times$ & \\
\hline & $\times$ & & $\times$ & $\times$ & & & & & & & & & & & $\times$ & $\times$ & & \\
\hline & $\times$ & & & & $\times$ & & & & $\times$ & $\times$ & & & & & & & & \\
\hline & & $\times$ & & $\times$ & $\times$ & $\times$ & & & & $\times$ & & & & & $\times$ & $\times$ & & \\
\hline & & & & & $\times$ & $\times$ & $\times$ & & $\times$ & & $\times$ & $\times$ & $\times$ & $\times$ & & & & $\times$ \\
\hline & $\times$ & $\times$ & $\times$ & $\times$ & $\times$ & & & $\times$ & & $\times$ & & & & & & & & \\
\hline 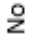 & $\stackrel{0}{2}$ & $\stackrel{0}{2}$ & $\stackrel{0}{2}$ & $\stackrel{\circ}{z}$ & $\stackrel{0}{2}$ & $\stackrel{\circ}{z}$ & $\stackrel{0}{2}$ & $\stackrel{0}{2}$ & 2 & $\stackrel{0}{2}$ & $\stackrel{\circ}{2}$ & $\stackrel{2}{2}$ & $\frac{0}{2}$ & $\stackrel{\circ}{2}$ & $\stackrel{0}{2}$ & $\stackrel{0}{2}$ & $\stackrel{0}{2}$ & 2 \\
\hline$\stackrel{y}{\check{y}}$ & $\stackrel{0}{2}$ & 울 & $\stackrel{0}{2}$ & $\stackrel{0}{z}$ & $\stackrel{0}{2}$ & $\stackrel{\circ}{z}$ & $\stackrel{\circ}{2}$ & $\stackrel{0}{2}$ & $\stackrel{\circ}{z}$ & $\stackrel{y}{\nu}$ & $\stackrel{y}{\check{2}}$ & $\stackrel{\circ}{2}$ & 을 & $\stackrel{\circ}{2}$ & $\stackrel{0}{2}$ & $\stackrel{y}{\rightleftharpoons}$ & $\stackrel{y}{\nu}$ & $\stackrel{\circ}{z}$ \\
\hline 总 & 岕 & 岕 & 岕 & 巡 & 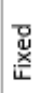 & 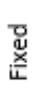 & $\begin{array}{l}\text { 밀 } \\
\text { x. }\end{array}$ & 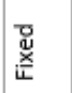 & 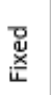 & 岕 & 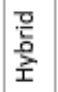 & 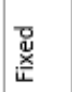 & $\begin{array}{l}\text { 은 } \\
\text { 童 } \\
\text { I }\end{array}$ & $\begin{array}{l}\text { D } \\
\text { 这 }\end{array}$ & 岕 & 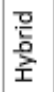 & 选 & $\begin{array}{l}\text { 莺 } \\
\text { 爻 }\end{array}$ \\
\hline 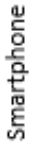 & 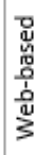 & 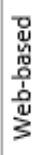 & 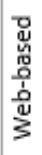 & 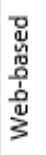 & 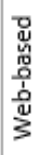 & 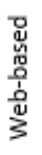 & 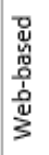 & 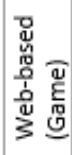 & 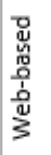 & 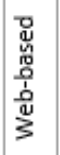 & 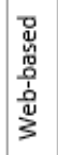 & 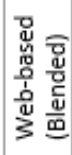 & 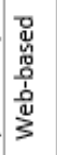 & 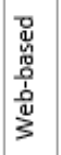 & 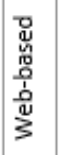 & 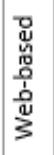 & 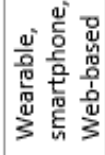 & $\begin{array}{l}0 \\
0 \\
0 \\
0 \\
0 \\
\vdots \\
\vdots \\
3 \\
3\end{array}$ \\
\hline 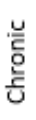 & 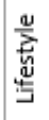 & 岂 & 亗 & 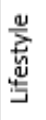 & 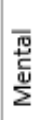 & 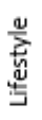 & 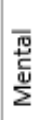 & 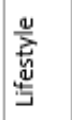 & 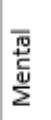 & 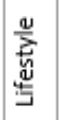 & 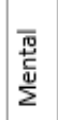 & 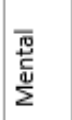 & 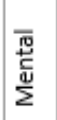 & 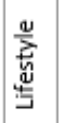 & 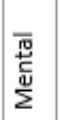 & 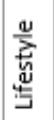 & 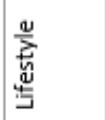 & 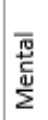 \\
\hline 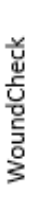 & 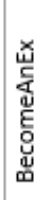 & 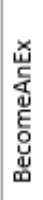 & 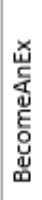 & 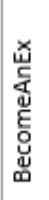 & 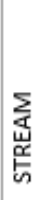 & 旁 & $\stackrel{\text { I0 }}{\mathrm{I}}$ & $\begin{array}{l}\frac{t}{\frac{1}{4}} \\
\frac{\pi}{0} \\
\frac{0}{0} \\
\frac{3}{4}\end{array}$ & $\stackrel{\mathfrak{m}}{\mathrm{I}}$ & $\stackrel{\mathbb{N}}{\mathrm{I}}$ & 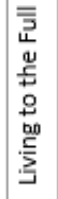 & $\stackrel{\mathscr{N}}{\mathrm{I}}$ & $\begin{array}{l}\xi \\
\frac{5}{J} \\
\frac{0}{2} \\
0 \\
\Sigma\end{array}$ & 岂 & 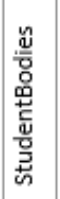 & 岁 & $\stackrel{\mathfrak{m}}{\mathrm{I}}$ & 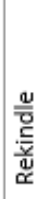 \\
\hline ิㅗํ & 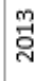 & $\stackrel{m}{3}$ & $\stackrel{2}{\circ}$ & ิㅗํ & 옹 & సิํำ & ন্ّ & $\stackrel{9}{\circ}$ & 올 & นี้ & $\stackrel{m}{\stackrel{n}{2}}$ & $\stackrel{\square}{\circ}$ & 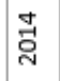 & ¿্่ & : & 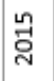 & $\stackrel{m}{\stackrel{D}{~}}$ & ठ্ণ \\
\hline 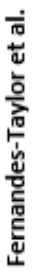 & 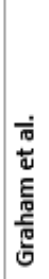 & 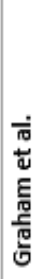 & 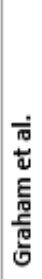 & 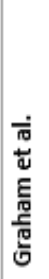 & 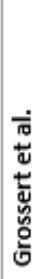 & 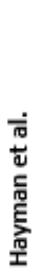 & $\begin{array}{l}\frac{j}{\pi} \\
\text { d̃ } \\
\text { 오 }\end{array}$ & 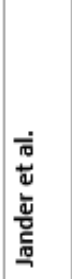 & 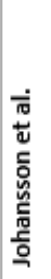 & 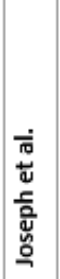 & 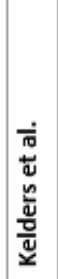 & 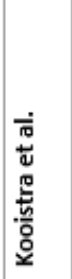 & 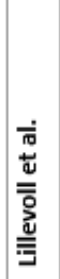 & 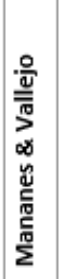 & 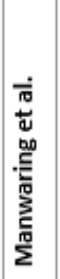 & 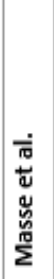 & 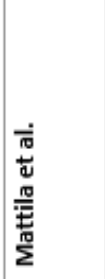 & 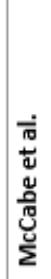 \\
\hline
\end{tabular}




\begin{tabular}{|c|c|c|c|c|c|c|c|c|c|c|c|c|c|c|c|c|}
\hline & & & & & & & & & & & & & $\times$ & $\times$ & $\times$ & \\
\hline & & & $x$ & & $\times$ & & & & $\times$ & & & & & & & \\
\hline & $\times$ & & & $\times$ & & $\times$ & & & & & & & & & & $\times$ \\
\hline & $\times$ & & $\times$ & & $\times$ & & $\times$ & & $\times$ & $\times$ & $\times$ & $\times$ & & & & $\times$ \\
\hline & & $\times$ & & & & & & & & & & & & & & \\
\hline$\stackrel{y}{\check{y}}$ & $\stackrel{2}{2}$ & $\stackrel{2}{2}$ & $\stackrel{2}{2}$ & $\stackrel{0}{2}$ & $\stackrel{2}{2}$ & $\stackrel{0}{2}$ & $\stackrel{2}{2}$ & $\stackrel{\circ}{z}$ & 을 & $\stackrel{0}{2}$ & 을 & $\stackrel{y}{\nu}$ & $\stackrel{\circ}{z}$ & 을 & $\stackrel{0}{2}$ & $\stackrel{0}{z}$ \\
\hline$\stackrel{y}{\check{y}}$ & 2 & 을 & 을 & 을 & $\stackrel{y}{\check{2}}$ & $\stackrel{y}{y}$ & 을 & $\stackrel{\circ}{2}$ & $\stackrel{y}{\simeq}$ & $\stackrel{\circ}{2}$ & 2 & $\stackrel{y}{\nu}$ & $\stackrel{y}{\check{y}}$ & $\stackrel{0}{\beth}$ & $\stackrel{y}{\searrow}$ & $\stackrel{9}{z}$ \\
\hline 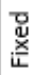 & $\begin{array}{l}\text { Zु } \\
\text { X } \\
\text { L }\end{array}$ & $\begin{array}{l}\text { 은 } \\
\text { 竞 }\end{array}$ & 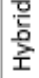 & 离 & 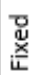 & 离 & $\begin{array}{l}\text { 은 } \\
\text { 咅 }\end{array}$ & 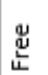 & $\begin{array}{l}\text { 离 } \\
\text { uxu }\end{array}$ & 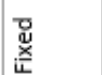 & 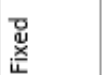 & 离 & $\begin{array}{l}\text { 은 } \\
\text { 졷 }\end{array}$ & 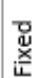 & 莺 & $\begin{array}{l}\text { 은 } \\
\text { 곧 }\end{array}$ \\
\hline 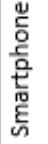 & 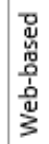 & 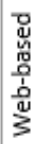 & 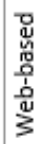 & 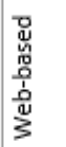 & 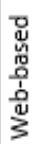 & 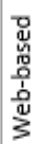 & 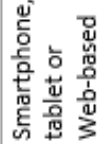 & 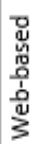 & 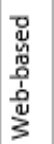 & 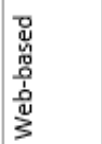 & 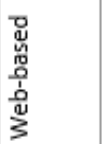 & 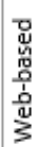 & 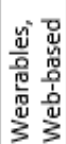 & 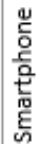 & 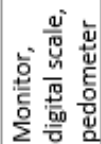 & 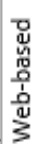 \\
\hline 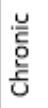 & 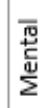 & 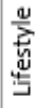 & 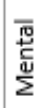 & 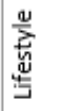 & 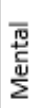 & 元 & 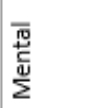 & 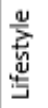 & 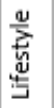 & 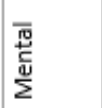 & 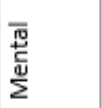 & 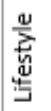 & 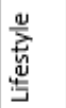 & 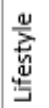 & 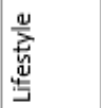 & 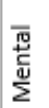 \\
\hline 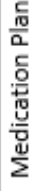 & 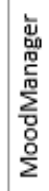 & 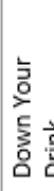 & 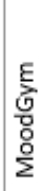 & 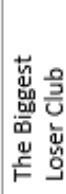 & $\stackrel{\mathbb{m}}{\mathrm{I}}$ & 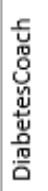 & 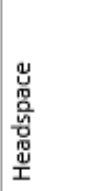 & $\stackrel{\text { I0 }}{=}$ & 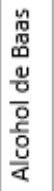 & 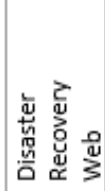 & 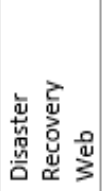 & $\stackrel{\text { I0 }}{\mathrm{C}}$ & 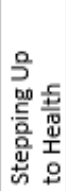 & $\stackrel{\mathbb{2 0}}{\mathrm{C}}$ & $\frac{\sum_{0}^{u}}{\frac{u}{2}}$ & $\stackrel{\text { IO }}{\mathrm{I}}$ \\
\hline 今ิ & त्र & 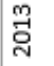 & ठั. & 옴 & 음 & न्: & 号 & 串 & ت्. & 苛 & సี & 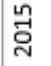 & 오․ & 号 & ने & 号 \\
\hline 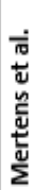 & 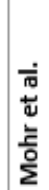 & 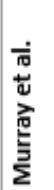 & $\begin{array}{l}\frac{10}{\pi} \\
\frac{d}{0} \\
\frac{0}{2}\end{array}$ & 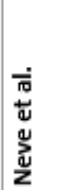 & 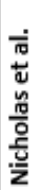 & 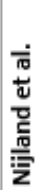 & 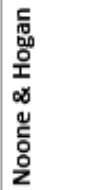 & 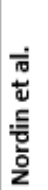 & 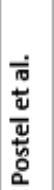 & 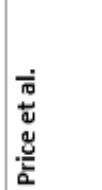 & 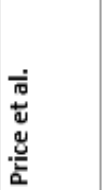 & 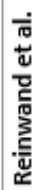 & 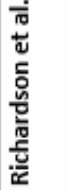 & 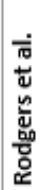 & 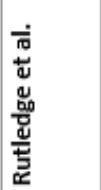 & 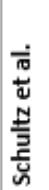 \\
\hline
\end{tabular}





\section{Chapter 3}

\section{Opening the Black Box of Electronic Health \\ Collecting, Analyzing, and Interpreting Log Data}

F. Sieverink, S. Kelders, M. Poel, J. van Gemert-Pijnen

JMIR Research Protocols, 2017;6(8):e156

DOI: $10.2196 /$ resprot.6452 


\section{Abstract}

Background: In eHealth research, limited insights have been obtained on process outcomes or how the use of technology has contributed to the users' ability to have a healthier life, improved wellbeing, or activate new attitudes in their daily tasks. As a result, eHealth is often perceived as a black box. To open this Black Box of eHealth, methodologies must extend beyond the classic effect evaluations. The analyses of log data (anonymous records of realtime actions performed by each user) can provide continuous and objective insights into the actual usage of the technology. However, until now the possibilities of log data in eHealth research has not been exploited to its fullest extent.

Objectives: The aim of this paper is to describe how log data can be used to improve the evaluation and understand the use of an eHealth technology with a broader approach than only descriptive statistics. This paper serves as a starting point for using log data analysis in eHealth research.

Methods: First, we describe what log data is and an overview is given of research questions to evaluate the system, the context, the users of a technology as well as the underpinning theoretical constructs. Secondly, requirements for log data, the starting points for the data preparation and methods for data collection are explained.

Results: In the third part, some methods for data analysis are described. Finally, a conclusion is drawn regarding the importance of the results for both scientific and practical applications.

Conclusion: The analysis of log data can be of great value for opening the black box of eHealth. A deliberate log data analysis can give new insights into how the usage of the technology contributed to the found effects and can thereby help to improve the persuasiveness and effectiveness of the eHealth technology and the underpinning behavioural models.

Keywords: eHealth, black box, evaluation, log data analysis 


\section{Introduction}

Many eHealth technologies, such as behaviour change technologies, aim to support users in reaching certain health-related behavioural outcomes. While such technologies can be effective [1, 2], one of the main problems is that users' adoption and long-term use remains lower than expected [3-5]. Moreover, eHealth research is dominated by a classic conception of medical research where randomized controlled trials (RCTs) are the golden standard for measuring outcomes [6]. Although a RCT provides valuable insights into the effectiveness of an intervention, fundamental to this methodology is to have the technology as a fixed entity for all participants throughout the whole intervention period. In contrast, (eHealth) technology can be characterized by its constant evolution and consequently, apps or interventions often become obsolete by the time the results of the RCT are available.

Furthermore, to conform to the complexity of behaviour change, eHealth technologies often consist of multiple components that may interact in reaching a certain effect and that people can use in many different ways in terms of the elements they use as well as the frequency, time and place of use $[7,8]$. RCTs however, do only provide insight into outcomes at fixed time points and treat technologies as a singular entity. Therefore, no insights can be obtained on process outcomes or how the use of the different components of the technology has contributed to healthier living, improved wellbeing, or a user's ability to conduct daily tasks $[7,9,10]$. This particular lack of insights is known as the 'Black Box Phenomenon' $[2,10,11]$. To open the Black Box of eHealth and to investigate why, how and for whom a certain technology is of the most value, methodologies must extend beyond the classic evaluations of effect only. In other words, the characteristics of eHealth technology change the way evaluations are conducted. In this view, Hekler and colleagues for example, plea for an 'agile science' approach that enables early and frequent insights into the process of behavior change via technology [7].

The CeHRes Roadmap (Figure 1) adopts an agile process in the development and evaluation process of eHealth technology. This roadmap is based on an extensive literature review of eHealth frameworks and follows a holistic and participatory research and development approach. Five phases can be distinguished in the development and evaluation of eHealth: (1) contextual inquiry, (2) value specification, (3) design, (4) operationalization, and (5) summative evaluation. Furthermore, the results of each phase should be the subject of formative evaluation, in order to collect input for improving the product [4].

According to the roadmap, technology development and evaluation is an iterative, flexible and dynamic process without a fixed endpoint. In this approach, continuous (formative and summative) evaluation is needed that is interwoven with all stages of technology development. The outcomes of such evaluations will be used for analyzing the process, recognizing the areas of improvement and diving deeper into the usage (the dose) that is 
needed to reach certain effects (the response). Thus, technology will already in early stages of development be reshaped by its usage. In order to do so, more advanced methods are needed to understand what people do with eHealth technology and how this is related to the impact.

The analysis of log data, defined as anonymous records of real-time actions performed by each user, has the potential to provide continuous and objective insights into the actual usage (of the different components) of the technology. Such analyses are therefore a promising approach to explain the outcomes of the more traditional methods such as RCTs, by gaining insight into the mediating mechanisms that contributed to the found effects $[8,9]$ and have also the potential to identify unexpected effects of a technology. The use of log data thus fits the aim of eHealth evaluation according to the CeHRes Roadmap by enabling early improvements of the technology in order to improve the evaluation outcomes.

Up to now, log data analyses are used in diversified domains, such as education [12, 13], human-computer interaction [14, 15], and network security [16]. Here, log data analyses are mainly used to analyse system performance and acceptance based on models for information retrieval. However, technology has evolved and the aim of behavior change technologies is not only to provide information, but rather to stimulate and support people in their process of behavior change [17]. Information is therefore needed regarding how the use of technology can explain the engagement and involvement of the individual user, the found effects and how the technology fits the user and the context.

Log data analyses in eHealth research have mainly focused on descriptive statistics, such as the number of logins, time spent and the frequency of use of the different elements by all users as a group (e.g., [18-20]). Although these statistics do provide valuable information regarding the usage of the technology, they also assume a dose-response relationship without taking the goal of the user into account. Furthermore, such analyses do not always provide insight into the actual process technology use in relation to behavior change. For example, a longer and more frequent exposure to an eHealth technology might indicate how well the system fits the users' needs, but it can also signify unfocused and/or non-strategic use or an inefficient system $[9,21]$. The same applies to only counting the number of logins, that say less when a user logs out directly and thus did not use the technology. We therefore need measures that indicate how the actual use of the (different elements of the) technology can explain the found effects.

To summarize, there is evidence that the use of log data can be of added value to the more traditional approaches of eHealth evaluation, but its possibilities have not yet been exploited to their fullest extent or have mostly been described on a conceptual level (e.g. [7-9]). The aim of this paper is therefore to give a more practical description of how log data can be of added value to understand the use of an eHealth technology with a broader approach than 
only descriptive statistics. In the following paragraphs we describe what research questions can be answered using log data. Furthermore, we will give directions on which steps need to be taken on the collection, preparation and analysis of log data, and how to interpret and apply the results (Figure 1 ).

The results and ideas as presented in this paper are substantiated with examples from prior log data research. These examples do not provide an exhaustive overview of all research conducted in this field but are used to illustrate the possibilities of log data as a starting point for further research to open the Black Box of eHealth.

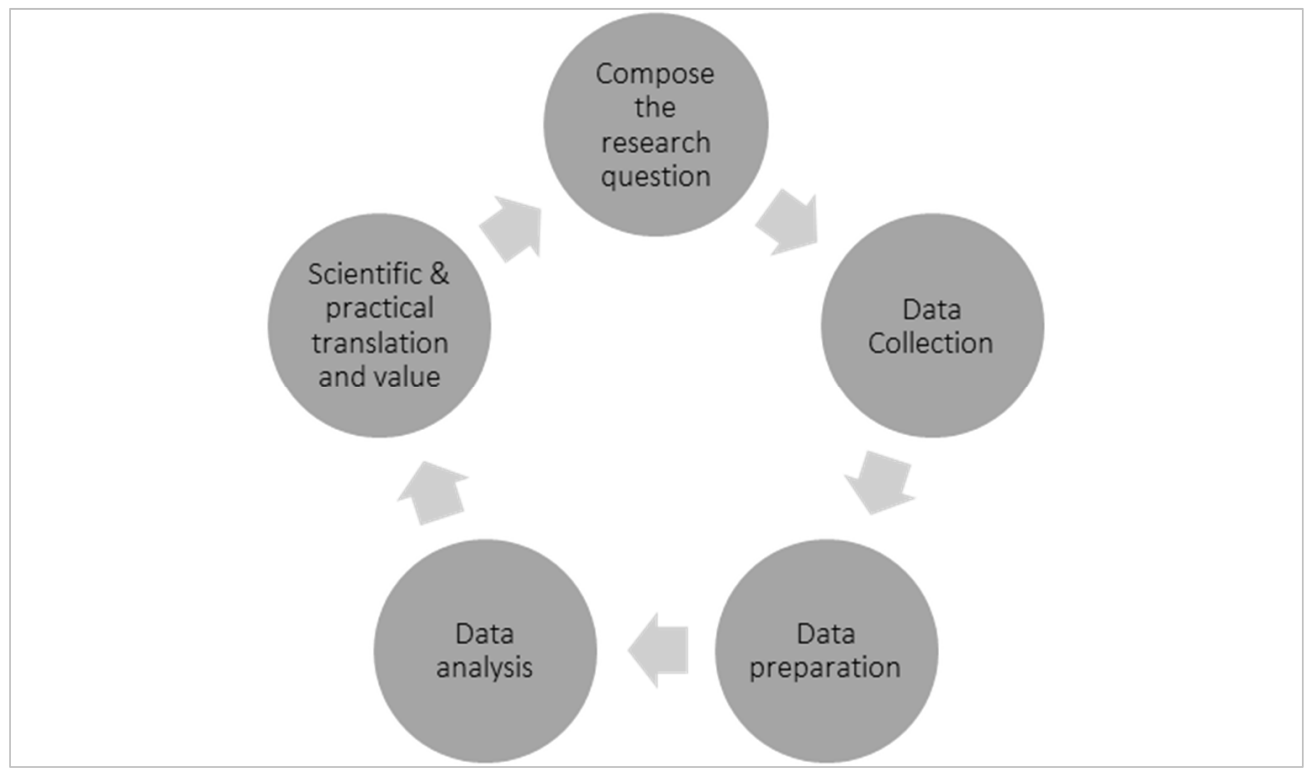

Figure 1. Steps in log data analysis

\section{Methods}

\section{What is log data?}

Generally (transactional) log data can be seen as behavioural logs that contain information regarding the interactions between a system and the users of that system $[9,14,15]$. These interactions can include information regarding time of the action, the content that is viewed or used, the mouse clicks, browsing patterns or saving information in the system. An important benefit of log data is that it represents the actual and continuous usage behaviour and not subjective, recalled behaviour.

Up to now, the use of log data mainly focused on the interaction with web-based technologies. However, eHealth technology has evolved from web- or telephone-based 
systems that required users' active involvement into unobtrusive and pervasive systems that are embedded into users' daily lives. For example, many people currently use lifestyle apps, such as 'Runkeeper' or food diaries, to support their daily routines. Wearable devices like the Fitbit, Jawbone or Apple Watch can continuously collect real-time health-related data for personalized coaching via smartphone or tablet applications. To be able to understand as much as possible of what people do with technologies or how they experience it, log data is not limited to the actual usage of web-based technologies anymore but it can include interactions with smartphone apps and wearables as well.

\section{Composing research questions}

Before the log data can be collected and analyzed, an important step is to revisit the goals of the technology and the subsequent research questions. A variety of research questions can be addressed with log data analysis, depending on the type and the goal of the eHealth technology and on the phase of development. According to the CeHRes Roadmap, log data analyses can be of added value in both the formative and summative evaluation phases [4].

Formative evaluation is conducted within and between the different phases of the roadmap. The aim of this type of evaluation is to check whether the goals of that phase have been reached. In the early operationalization phase for example, log data has the potential to evaluate the use of the system and to assess what people do (or not do) with a technology. Critical moments for dropping out can be identified, as well as profiles for both users and usage. The results from these evaluation can be used to improve an early release of the technology before it will be available for a bigger group of users, which fits the 'agile science' approach [7]. Possible research questions for formative system evaluation are:

- What usage patterns emerge when users navigate through the technology?

- Which (combinations of) elements of the technology are used?

- When do users drop out?

- How do users respond to behavior change strategies and persuasive triggers (strategies to support users in performing certain (usage) behaviors and/or long-term use, such as reminders)?

In a previous study we found for example that users of a Personal Health Record (PHR), an electronic application consisting of different tools for monitoring and coaching patients with chronic conditions to support self-management [22] follow the global menu structure when exploring the PHR for the first time. Furthermore, most users were likely to drop out when they visited the education section as a first step after the first login [23].

The uptake and impact of a technology are measured during the summative evaluation phase. With the impact we refer to whether the intended goals of the technology have been realized in terms of behavioral, clinical and organizational outcomes. The uptake refers to 
the implementation and usage of the technology. Log data can be used to assess the uptake of the technology. Where a log data analysis in the operationalization phase mainly focuses on the system performance, in this phase research questions to evaluate the whole of the system, the user and the context can be formulated. Possible research question to evaluate the system are:

- How do users use the technology in order to complete an intervention or to achieve their health-related goals (in terms of frequency or combinations of elements they use)?

- How well do the users adhere to the intended usage of the technology?

- What are predictors for adherence or dropping-out?

- How does the use of the technology change over time?

- How did these usage patterns contribute to the (clinical, behavioural and organizational) impact? In other words: what is the dose-response relationship?

Studies of Kelders et al. [21] and Van Gemert et al. [24] for example, showed at what stages of a web-based intervention for the early treatment of depressive symptoms support ("Living to the Full") it was relevant to prevent users from dropping out from that intervention. Another example comes from a study of Freyne et al. [25], who found that uploading a profile picture on a diet support site in the first week, resulted in higher return rates. Research questions to evaluate the users of the technology are:

- Who is motivated and capable of using the eHealth technology?

- Who are the long-term users?

- Who are the drop-outs?

Possible context-related research questions are:

- How does the responsiveness of caregivers (e.g., time until replying to a users' message) influence the use of eHealth technology by patients?

- How does the technology integrate into users' daily lives?

Log data analyses can provide answers to more fundamental research questions as well, for example to test existing models and theories [8]. Behaviour change theories and behaviour models often form the basis for the content and the structure of eHealth technologies. For example, mental health interventions are often based on the principles of cognitive behavioural therapy and principles from the goal setting theory are used to support users in reaching their health-related goals. Log data can be used to check whether the incorporated (combination of) elements that represent certain theoretical concepts (e.g., a chat box to facilitate social support) have been used. Or, when one of the goals of a technology is e.g. to improve self-efficacy, log data can provide insight into what the most effective usage 
patterns are and for whom to experience any improvements in the self-efficacy. This results in the following research questions:

- To what extent did the users find and use the (combination of) elements of the technology that represent certain theoretical concepts?

- How did the use of (a combination of) these elements contributed to any improvements in the outcomes?

The "Living to the Full" intervention for example, is based on the principles of acceptance and commitment therapy (ACT), where mindfulness is an important part of. An effect evaluation showed positive results, indicating that a web-based technology based on ACT might help users in reducing depressive complaints [26]. A log data analysis however, revealed that many users of that intervention did not open the mindfulness exercises that are assumed to be an important element of ACT [24]. This might be an indicator that the concept of mindfulness is insufficiently operationalized in the intervention and that the found effects are an underestimation of the attainable effects.

While not complete or exhaustive, this overview serves as a starting point for composing suitable research questions for a holistic and agile evaluation eHealth technology. The proposed questions can be adjusted based on the goal of the technology and the incorporated behavioural models and/or theories. The answers to the research questions can provide input for improving the look and feel and the architecture of the technology as well as the fit between the technology, the user and the context. In turn, this information can be used to increase the effectiveness, persuasiveness and the long-term usage of the technology.

\section{Data collection}

Depending on the research questions, there are different ways to collect log data. To gain rich and in-depth knowledge regarding the usage patterns of individual users, server-side log data, containing information about communications with the server (requests such as opening a page, clicking a link, saving health values or other information), can be collected. In web-based applications, this type of logging is preferably a file where the web addresses of the requested subpages of the system are registered. This is the most efficient way without substantially losing system performance. Another advantage is that, after updates and modifications of the system, web addresses referring to new subpages and functions are automatically logged.

On the other side, a single user action (e.g. clicking a button to add a health measurement) can lead to multiple server requests, leading to multiple web addresses in the log data file. This can make it harder to identify single user actions. It is therefore necessary to determine 
which (combinations of) server requests specify certain actions and to link these to a specific identification for that action, such as a code or description.

Besides server-side logging, client-side logging can as well provide valuable information regarding the usage of the technology. Client-side information contains actions that do not require server requests, such as scrolling up and down the screen, moving the pointer, and clicking and filling out a text field. The research questions determine the logging method that provides the most valuable information. However, it is also important to take the possibilities and the consequences of the different logging methods into account, such as a loss of system performance.

\section{Requirements of log data}

Log data files are most often Comma-Separated Values (.csv) files that can easily be opened using Excel or SPSS. Information regarding the user identity, date and time of the action and an identification of the action is essential to identify the user, logins and the usage patterns within and between logins. Figure 2 shows an example of a fictional log data file. Depending on the research questions, additional information can be desirable regarding the device (e.g., PC, smart phone, tablet, or wearable), a specification of the action (e.g., the measurements or other information that is saved in the database), the GPS coordinates of the user, but also the status of the user collected via wearables, such as stress, sleep, and activity patterns.

\begin{tabular}{|llll|}
\hline User & Time Stamp & Action & Extra information \\
\hline $\mathbf{1}$ & January $12 ; 01: 14$ p.m. & Login & \\
\hline $\mathbf{1}$ & January $12 ; 01: 21$ p.m. & Login & \\
\hline $\mathbf{2}$ & January $12 ; 01: 20$ p.m. & Login & \\
\hline $\mathbf{2}$ & January $12 ; 01: 22$ p.m. & Opening monitoring & Blood pressure \\
\hline $\mathbf{2}$ & January $12 ; 01: 47$ p.m. & Adding monitoring value & \\
\hline $\mathbf{3}$ & January $21 ; 10: 11$ a.m. & Login & \\
\hline $\mathbf{3}$ & January $21 ; 10: 12$ a.m. & Opening mailbox & \\
\hline $\mathbf{3}$ & January $21 ; 10: 13$ a.m. & Opening monitoring & To general practitioner \\
\hline $\mathbf{3}$ & January 21; 10:13 a.m. & Opening mailbox & \\
\hline $\mathbf{3}$ & January 21; 10:21 a.m. & Send message & To nurse practitioner \\
\hline $\mathbf{1}$ & January 23; 10:11 a.m. & Login & \\
\hline $\mathbf{1}$ & January 23; 10:13 a.m. & Opening mailbox & \\
\hline $\mathbf{1}$ & January 23; 10:15 a.m. & Send message & \\
\hline
\end{tabular}

Figure 2. A fictional example of log data 
In order to answer the research questions, data files should be of sufficient quality, wherein the goal of the technology and the used behaviour change theories and models form the basis for the data that is needed for the analyses. For example, if the research question is "What are predictors for adherence to the technology?" then the data should contain information from which the adherence can be derived, as well as the variables (such as user or usage characteristics) that might possibly predict adherence. When the focus is on exploring the dose-response relationship, there must be a possibility to link the log data to other outcomes, for instance via the user identification number.

The amount of data needed depends on the complexity of the research question and can only be determined empirically. In general, a reasonable amount of data per user and a reasonable amount of users are needed in the dataset. When analysing 100 usage sessions for example, 10 usage sessions of 10 different users provide more generalizable information than 50 usage sessions of two different users. Of course, the more data the better, but it needs to contain the needed information to answer the research question as well.

Importantly, the data should be available for analysis under the applicable privacy regulations, whether or not with informed consent of the individual users of the technology. Informed consent depends upon whether log data includes or needs to be combined with personal data. Currently, it remains undecided whether log data in itself is personal data, as usage data only does not, per se, contain information that can be traced back to individual users. However, as the possibilities for data analytics develop, it may become quite possible in the (near) future to trace users back to specific individuals based on their usage patterns on other technologies. Narayanan \& Shmatikov for example, were able to de-anonymize Netflix users based on reviews in the Internet Movie Database (IMDB) [27] and it can thus be hard to truly anonymize log data. Therefore researchers need to always consider the applicable regulations to determine whether informed consent is needed from the users before log data can be collected and analysed for research purposes.

\section{Data preparation}

Preparing the data before analysis is vital since, for the most part, typical log data consist of tens of thousands records. Hence, these records must be first filtered for the information that is needed for the analysis (e.g., the web addresses or the codes for specific actions). Then this information needs to be translated into new variables, such as the number of sessions and/or activities per user, sequence of the activities, and/or time spent per login. Figure 3 shows an example of how the raw data as shown in Figure 2 is translated into data for analyses. There are different variables that can be calculated.

In Figure 3, every row in the new data set represents one user. The number of sessions is defined as a period of activity ended by a period of at least 30 minutes of inactivity. In this example, this definition has consequences for User 1 , having three logins and two sessions. 
By counting the number of logins it would seem like this user has a higher activity level then by counting the number of sessions. It is well possible however, that a user picks up the activities where he left off when returning within 30 minutes after the last action. Furthermore, no user actions were registered during the first and the second login. Hence, counting the number of logins (only) might give a distorted image on the amount of actual use of the different elements of technology.

Second, a distinction was made in this example between visiting a certain element of the technology and actually using it (e.g., opening monitoring and adding a value, or opening the mailbox and sending a message). Thus, two out of the three users in this example have opened the function for monitoring, but only one of these users has actually used this function by adding a value to the database. Furthermore, two out of three users have opened their mailboxes and sent a message to a caregiver, where User 3 opened the mailbox twice but sent a message once.

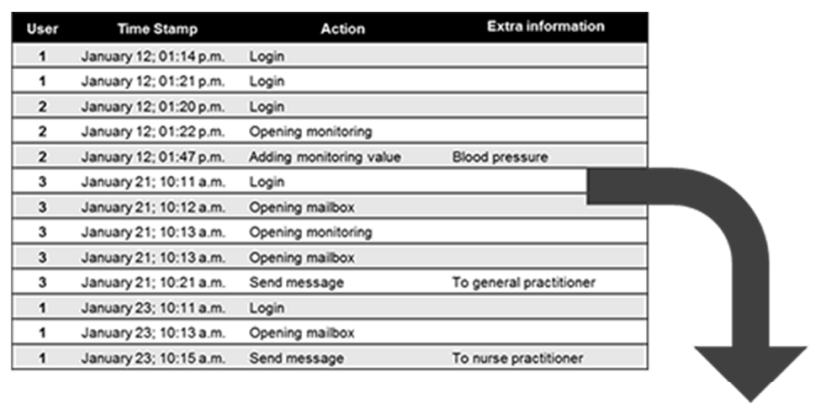

\begin{tabular}{|cccccc|}
\hline User & \#Sessions & $\begin{array}{c}\text { \# Monitoring } \\
\text { opened }\end{array}$ & $\begin{array}{c}\text { \# Values } \\
\text { added }\end{array}$ & $\begin{array}{c}\text { \# Mailbox } \\
\text { opened }\end{array}$ & \# Messages sent \\
\hline 1 & 2 & 0 & 0 & 1 & 1 \\
\hline 2 & 1 & 1 & 1 & 0 & 1 \\
\hline 3 & 1 & 1 & 0 & 2 & 0 \\
\hline
\end{tabular}

Figure 3: An example of data transformation, based on Figure 2

These are only a few examples of the variables that can be calculated from a raw log data set. Depending on the research questions, new or other variables can be calculated as well. When the question is for instance how the usage in the first sessions correlates to adherence, a distinction can be made between the activities (e.g., opening the mailbox and sending a message) in the first, second, third, and further sessions. Also, variables can be added regarding the adherence by a user (where an adherent user is indicated with a ' 1 ' and a nonadherent user with a ' 0 '); whether the sent message has been answered (and within what time period); the location of the user (where GPS coordinates are given for every session); 
the emotional status of the user; activity levels; or the time (in days or hours) between the previous and the current sessions. By combining different data sources, new variables do not have to contain, by definition, information from the same data file.

\section{Data analysis}

Once the log datasets are prepared, the files are ready for analysis. The first and up to now most commonly used method is to describe the frequencies of use, including: the number of logins or sessions per user, the use of the different elements of the technology, the moment of use, and the time spent on the technology. Although more exposure to an eHealth technology does not always lead to better health outcomes, this information might still provide a starting point for further research. Next, pathway analyses and predictive modelling provide deeper insights into the usage patterns of individual users.

A pathway analysis can provide more information regarding the different usage patterns that occur. In previous research, for example, the usage patterns of adherers and early and late non-adherers to the "Living to the Full" intervention were compared [21]. A 1-way analyses of variance (ANOVA) and chi-square tests (X2) showed that early non-adherers used fewer and shorter sessions than late non-adherers and adherers. Early non-adherers also used fewer sessions to complete a lesson. Furthermore, late non-adherers had a shorter total duration of sessions than the adherers. Logistic regression was used to assess the baseline characteristics of adherers and non-adherers.

We also have analysed usage patterns of first visits to the PHR for patients with chronic conditions [23]. The results showed that users followed the structure of the system. While these analyses were conducted by hand on a subset of all users, for analysing the dominant path through the system of a more extended group of users, Markov modelling methods can be more convenient $[28,29]$. This methodology can be used, for example, to analyse how people use the different elements of a technology in terms of frequency and the order they select to reach a certain goal [30].

More advanced and predictive information for pattern recognition within complex data sets can be obtained by applying machine-learning algorithms [31]. To do so, the Waikato Environment for Knowledge Analysis (Weka) tool is a relatively accessible and easy to use software package for applying machine learning algorithms for data mining tasks [32]. By using Weka, the following methods for analysis can be applied: 
- Supervised learning. Predicts adherence and effects from early use patterns, which enables early intervention for users at risk [31,33,34]. This method has the potential to answer research questions concerning when users drop out and what the predictors are for users dropping out or returning to the application.

- Unsupervised learning. Determines what usage profiles appear from the log data and if this data can be matched to a certain group of participants [33, 35]. This method has the potential to answer the research questions: What are the characteristics of (non)users, and who are the dropouts?

- Market-basket analysis. This method allows researchers to ask what elements of the technology are often used together [36]. Examples of such analyses can be found in the domain of online shopping, where customers get to see suggestions of products based on the products they looked at.

Although it is difficult to make predictions based on the usage data of relatively small groups of users (e.g., in a RCT) and not all research questions can be answered with this data, even these analyses can provide valuable scientific and practical input for future system improvements.

\section{Discussion}

Log data analyses can be used as part of the formative as well as the summative evaluation of eHealth technology. As a formative evaluation, log data can provide ongoing and real-time information on how to improve the technology and on the process in which the technology is embedded. As part of the summative evaluation, log data can provide explanations on the uptake and the outcomes of the technology, which can be both scientifically and practically valuable.

\section{Scientific translation}

As stated in the Introduction, log data analyses provide input for opening the Black Box of eHealth. Log data analysis not only provides insights into the effects of the single elements of a technology, but can also stipulate essential information about the effects of combinations of elements. In this way, log data can provide input to better understand the results of experimental research designs, such as RCTs or the Multiphase Optimization Strategy (MOST) for eHealth evaluation. In a MOST, potential effective elements are selected for incorporation in an intervention (based on existing theories and/or previous research) and tested in three subsequent phases (screening phase, refining phase and the confirming phase) [37]. Log data has the potential to validate the results of a RCT or these different phases of the MOST. For example, did the users actually find all the incorporated elements of the technology and are these elements used in the intended way? [24] And how does the use of the different elements correlate to the found effects, and for whom? Based on such 
insights, existing technologies can be improved and effective elements can be identified and combined into new technologies. These results are not always revealed through questionnaires, interviews, or usability tests.

Another advantage of a log data analysis is that it can reveal real-time insights into the user's response to specific persuasive triggers in different situations (e.g., in terms of location, status of the user), providing new possibilities for the timing of persuasion [38]. Furthermore, several studies have demonstrated that individuals respond differently to the same persuasive strategies [39], indicating that personalization of a technology (adapting a technology to individual users) might increase a program's persuasiveness and thus its longterm use and effectiveness [40].

In the domain of eHealth, personalization is often limited to adapting the content of the technology to a confined user profile based on user characteristics like age, gender and level of education [41]. However, there is evidence that such demographics (user profiles) do not predict engagement [42]. Usage profiles, such as early usage patterns for example, do potentially predict whether or not an individual will maintain long-term use of an application. For example, Freyne et al. [25] found that individuals' use of specific elements of a technology in the first week influences their use of that technology in the second week. Based upon these results, more extended user profiles can be created that take (early) usage behaviour into account and thus extending user profiles beyond a limited set of user characteristics.

Log data analyses also allow a timely response to flaws in the technology, a shorter evaluation cycle, and the development of more transparent technology, as stressed in the 'agile science' approach [7]. Until now, modifications of technologies have often been made after an evaluation period. However, technology use is often not stagnant and changes over time and we therefore need statistics that fit with this characteristic [43]. With a real-time analysis of log data, adaptive interfaces can be created that respond to individual (changes in) usage patterns. The effects of these adaptive interfaces on the usage can then be analysed further and improved.

Log data can as well be used to test the models and theories that are incorporated in the technology in order to improve the existing behavioural models. Patrick and his colleagues make the following comparison for this approach: "It could be argued that today's current behavioral theories are akin to the Farmer's Almanac as they are largely descriptive, pastoriented, and simplified to a few elements. These models for understanding behavior and behavior change provide largely "on average" insights without the level of specification and prediction that could occur in behavioral science if the approach to communication, data, and iterative evaluation of computationally complex, multilevel models now common in meteorology could be replicated" [31], p. 819. Log data has the potential to predict usage 
behaviour and can thus be of added value in the development of complex, multilevel models for behaviour change.

Furthermore, machine-learning algorithms can, for instance, make predictions regarding whether and when a user might dropout from using the technology, making it possible to intervene in a timely manner and thus to increase adherence to the technology. However, when focusing on research questions to assess adherence, it is important to substantiate this term: when is a user considered to be adherent? In many research, assumptions are made about the intended usage of a technology which are not well defined or evidence-based [4446]. As a result, it is hard to assess the results of the analyses and compare those to the outcomes of other, similar technologies. A definition of adherence does not always have to be derived from the extent to which a technology is completed (e.g., a user is adherent when he/she completed 3 out of 4 lessons), but can also be extracted from other literature. For example, Kaushal and Rhodes discovered that exercising for at least four times a week for six weeks was the minimum activity to establish an exercise habit [47]. This type of evidence can well be used for defining adherence to a technology, e.g., a user is adherent when at least four usage sessions per week can be identified for a period of six weeks. In this example, mere login data (e.g., the more logins, the better the adherence is) does not reveal adherence, but assigning a substantiated threshold value does.

An advantage of log data is that it is always available and easy to collect, without requiring any extra effort from the participants. A common problem in (eHealth) research is that participants often find it time consuming and labour intensive to complete questionnaires at different time points or to participate in an interview or focus group, resulting in dropouts from the research study. However, this result does not necessarily mean that the same participants who did not participate in the research dropped out from using the technology as well. By using log data in addition to questionnaires, researchers have more than one method to collect data, and are no longer dependent on having a majority of the participants complete questionnaires or participate in interviews or focus groups.

However, there are important limitations for using log data in eHealth evaluation as well. First, the results of the log data analyses in itself do not always indicate why certain usage patterns occur. It is therefore important to combine the analyses with additional research via interviews, usability tests, or other quantitative and qualitative research methods. For example, the log data analysis from the "Living to the Full" intervention showed that a fairly large group stopped to adhere during the sixth lesson. A check among the counsellors who have given this course revealed that this is indeed a hard lesson for participants, because of the focus the observing self and learning new skills to accept suffering [21]. Additional research can thus provide more precise insights into what users experience or why they tend to drop out at certain points. Log data analysis focused on such questions can thus provide 
researchers specific areas or user groups to examine through future interviews, questionnaires, or usability tests. The results of these evaluations can then be used to improve the technology as well as to highlight the crucial moments in the treatment protocols for blended therapy.

Furthermore, using log data in research might require an extra effort from researchers, developers, database managers, et cetera. For example, it takes time to develop a plan for data collection, management and analysis, as well as to incorporate the possibility for data collection into the technology.

\section{Practical value}

Besides the scientific value, the results of a log data analysis can be of added value for eHealth developers and healthcare providers. For example, the results of a pathway analysis and the identified usage profiles can be used as input for adapting and matching the system design to the users, in order to make the technology more persuasive. Information regarding the elements that are often used together can also provide real-time feedback and suggestions to the users, guiding their follow-up actions in the system (e.g., "You have added a goal. Other users have added their current weight as well. Click here to add your weight.")

Because log data analysis via (un)supervised learning can provide information about users that might potentially drop out from an intervention, on a practical level, healthcare providers can then make use of this information to intervene and stimulate these users to continue using the system. In addition, log data can be used to show healthcare providers how their responsiveness to client messages influences a client's adherence to the therapy. When composing protocols for (blended) care via eHealth technologies, researchers can then take advantage of the added value of log data analyses.

Until recently, technologies have often changed after an evaluation period, but with a realtime analysis of log data, adaptive interfaces can be created that respond to individual users. The effects of the interface on the use of the technology can then be directly identified, allowing a fast response to flaws in the technology, a shorter evaluation cycle and the development of more transparent technology.

\section{Conclusion}

The analysis of log data can be of great value for scientists and designers as well as caregivers and policy makers in their research into the black box of eHealth technology. A deliberate analysis of log data can provide insights into the usage of the technology by all users as a group as well as by individual users, helping to accelerate the persuasiveness and effectiveness of eHealth technology. Furthermore, log data can be used to assess the theories that underpin a technology. However, from the collection of log data to translating 
the results into valuable information, various steps need to be taken, each with their own considerations. This paper serves as a starting point for using log data analysis in eHealth research. 


\section{References}

1. Oinas-Kukkonen H, Harjumaa M. Persuasive systems design: Key issues, process model, and system features. Communications of the Association for Information Systems. 2009;24(1):28.

2. Kelders SM, Oinas-Kukkonen H, Oörni A, van Gemert-Pijnen JEWC. Health Behavior Change Support Systems as a research discipline; A viewpoint. International Journal of Medical Informatics. 2016;96:3-10.

3. Nijland N, van Gemert-Pijnen JE, Kelders SM, Brandenburg BJ, Seydel ER. Factors Influencing the Use of a Web-Based Application for Supporting the Self-Care of Patients with Type 2 Diabetes: A Longitudinal Study. J Med Internet Res. 2011;13(3):e71.

4. Van Gemert-Pijnen JE, Nijland N, van Limburg M, Ossebaard HC, Kelders SM, Eysenbach G, et al. A Holistic Framework to Improve the Uptake and Impact of eHealth Technologies. J Med Internet Res. 2011;13(4):e111.

5. Black AD, Car J, Pagliari C, Anandan C, Cresswell K, Bokun T, et al. The Impact of eHealth on the Quality and Safety of Health Care: A Systematic Overview. PLOS Medicine. 2011;8(1):e1000387.

6. Eysenbach G. CONSORT-EHEALTH: Improving and Standardizing Evaluation Reports of Web-based and Mobile Health Interventions. J Med Internet Res. 2011;13(4):e126.

7. Hekler EB, Klasnja P, Riley WT, Buman MP, Huberty J, Rivera DE, et al. Agile science: creating useful products for behavior change in the real world. Translational Behavioral Medicine. 2016;6(2):31728.

8. Moller AC, Merchant G, Conroy DE, West R, Hekler E, Kugler KC, et al. Applying and advancing behavior change theories and techniques in the context of a digital health revolution: proposals for more effectively realizing untapped potential. Journal of Behavioral Medicine. 2017;40(1):85-98.

9. Han JY. Transaction logfile analysis in health communication research: Challenges and opportunities. Patient Education and Counseling. 2011;82(3):307-12.

10. Resnicow K, Strecher V, Couper M, Chua H, Little R, Nair V, et al. Methodologic and Design Issues in Patient-Centered e-Health Research. American Journal of Preventive Medicine. 2010;38(1):98102.

11. van Gemert-Pijnen J, Peters O, Ossebaard HC. Improving eHealth: Eleven international publishing Den Haag, The Netherlands; 2013. ISBN: 9462360677.

12. Bruckman A. Analysis of Log File Data to Understand Behavior and Learning in an Online Community. In: Weiss J, Nolan J, Hunsinger J, Trifonas P, editors. The International Handbook of Virtual Learning Environments. Dordrecht: Springer Netherlands; 2006. p. 1449-65.

13. Jamali HR, Nicholas D, Huntington P. The use and users of scholarly e-journals: A review of log analysis studies. Aslib Proceedings. 2005;57(6):554-71.

14. Dumais S, Jeffries R, Russell DM, Tang D, Teevan J. Understanding User Behavior Through Log Data and Analysis. In: Olson JS, Kellogg WA, editors. Ways of Knowing in $\mathrm{HCl}$. New York, NY: Springer New York; 2014. p. 349-72.

15. Jansen BJ. Search log analysis: What it is, what's been done, how to do it. Library \& Information Science Research. 2006;28(3):407-32.

16. Choi Y, Chang S, Kim Y, Lee H, Son W, Jin S. Detecting and monitoring game bots based on largescale user-behavior log data analysis in multiplayer online games. The Journal of Supercomputing. 2016;72(9):3572-87. 
17. Chatterjee S, Price A. Healthy Living with Persuasive Technologies: Framework, Issues, and Challenges. Journal of the American Medical Informatics Association. 2009;16(2):171-8.

18. Glasgow RE, Christiansen SM, Kurz D, King DK, Woolley T, Faber AJ, et al. Engagement in a Diabetes Self-management Website: Usage Patterns and Generalizability of Program Use. J Med Internet Res. 2011;13(1):e9.

19. Kim E-H, Stolyar A, Lober WB, Herbaugh AL, Shinstrom SE, Zierler BK, et al. Usage patterns of a personal health record by elderly and disabled users. Annual Symposium proceedings AMIA Symposium. 2007:409-13.

20. Kuijpers W, Groen WG, Oldenburg HS, Wouters MW, Aaronson NK, van Harten WH. eHealth for Breast Cancer Survivors: Use, Feasibility and Impact of an Interactive Portal. JMIR Cancer. 2016;2(1):e3.

21. Kelders SM, Bohlmeijer ET, Van Gemert-Pijnen JE. Participants, Usage, and Use Patterns of a WebBased Intervention for the Prevention of Depression Within a Randomized Controlled Trial. J Med Internet Res. 2013;15(8):e172.

22. Pagliari C, Detmer D, Singleton P. Potential of electronic personal health records. BMJ. 2007;335(7615):330-3.

23. Sieverink F, Kelders SM, Braakman-Jansen LMA, Gemert-Pijnen JEWCV. The Added Value of Log File Analyses of the Use of a Personal Health Record for Patients With Type 2 Diabetes Mellitus. Journal of Diabetes Science and Technology. 2014;8(2):247-55.

24. Van Gemert-Pijnen JE, Kelders SM, Bohlmeijer ET. Understanding the Usage of Content in a Mental Health Intervention for Depression: An Analysis of Log Data. J Med Internet Res. 2014;16(1):e27.

25. Freyne J, Saunders I, Brindal E, Berkovsky S, Smith G. Factors associated with persistent participation in an online diet intervention. $\mathrm{CHI}$ '12 Extended Abstracts on Human Factors in Computing Systems; Austin, Texas, USA: ACM; 2012. p. 2375-80.

26. Bohlmeijer ET, Fledderus M, Rokx TAJJ, Pieterse ME. Efficacy of an early intervention based on acceptance and commitment therapy for adults with depressive symptomatology: Evaluation in a randomized controlled trial. Behaviour Research and Therapy. 2011 1//;49(1):62-7.

27. Narayanan A, Shmatikov V, editors. Robust De-anonymization of Large Sparse Datasets. 2008 IEEE Symposium on Security and Privacy (sp 2008); 2008 18-22 May 2008.

28. Seneta E. Markov and the Birth of Chain Dependence Theory. International Statistical Review / Revue Internationale de Statistique. 1996;64(3):255-63.

29. Borges J, Levene M. Evaluating Variable-Length Markov Chain Models for Analysis of User Web Navigation Sessions. IEEE Transactions on Knowledge and Data Engineering. 2007;19(4):441-52.

30. Akkersdijk SM, Kelders SM, Jansen LMAB, Pijnen LvG, editors. Using Markov Chains to analyze paths through a Personal Health Record. Persuasive Technology XII (Adjunct Proceedings); 2017 April 47, 2017; Amsterdam.

31. Patrick K, Hekler EB, Estrin D, Mohr DC, Riper H, Crane D, et al. The Pace of Technologic Change. American Journal of Preventive Medicine. 2016;51(5):816-24.

32. Hall M, Frank E, Holmes $G$, Pfahringer B, Reutemann P, Witten IH. The WEKA data mining software: an update. SIGKDD Explor Newsl. 2009;11(1):10-8.

33. Han J, Pei J, Kamber M. Data mining: concepts and techniques: Elsevier; 2011. ISBN: 0123814804. 
34. Hekler EB, Michie S, Pavel M, Rivera DE, Collins LM, Jimison HB, et al. Advancing Models and Theories for Digital Behavior Change Interventions. American Journal of Preventive Medicine. 2016 11//;51(5):825-32.

35. Paliouras G, Papatheodorou C, Karkaletsis V, Spyropoulos CD. Discovering user communities on the Internet using unsupervised machine learning techniques. Interacting with Computers. 2002;14(6):761-91.

36. Anand SS, Patrick AR, Hughes JG, Bell DA. A Data Mining methodology for cross-sales. KnowledgeBased Systems. 1998;10(7):449-61.

37. Collins LM, Murphy SA, Strecher V. The Multiphase Optimization Strategy (MOST) and the Sequential Multiple Assignment Randomized Trial (SMART): New Methods for More Potent eHealth Interventions. American Journal of Preventive Medicine. 2007;32(5):S112-S8.

38. Räisänen T, Oinas-Kukkonen H, Pahnila S. Finding Kairos in Quitting Smoking: Smokers' Perceptions of Warning Pictures. In: Oinas-Kukkonen H, Hasle P, Harjumaa M, Segerståhl K, Øhrstrøm P, editors. Persuasive Technology: Third International Conference, PERSUASIVE 2008, Oulu, Finland, June 4-6, 2008 Proceedings. Berlin, Heidelberg: Springer Berlin Heidelberg; 2008. p. 254-7.

39. Kaptein M, Eckles D. Heterogeneity in the Effects of Online Persuasion. Journal of Interactive Marketing. 2012;26(3):176-88.

40. Kaptein M, Markopoulos P, de Ruyter B, Aarts E. Personalizing persuasive technologies: Explicit and implicit personalization using persuasion profiles. International Journal of Human-Computer Studies. 2015;77:38-51.

41. Kaptein M, Lacroix J, Saini P. Individual Differences in Persuadability in the Health Promotion Domain. In: Ploug T, Hasle P, Oinas-Kukkonen H, editors. Persuasive Technology: 5th International Conference, PERSUASIVE 2010, Copenhagen, Denmark, June 7-10, 2010 Proceedings. Berlin, Heidelberg: Springer Berlin Heidelberg; 2010. p. 94-105.

42. van Mierlo T, Li X, Hyatt D, Ching AT. Demographic and Indication-Specific Characteristics Have Limited Association With Social Network Engagement: Evidence From 24,954 Members of Four Health Care Support Groups. J Med Internet Res. 2017;19(2):e40.

43. Magni M, Susan Taylor M, Venkatesh V. 'To play or not to play': A cross-temporal investigation using hedonic and instrumental perspectives to explain user intentions to explore a technology. International Journal of Human-Computer Studies. 2010;68(9):572-88.

44. Kelders SM, Kok RN, Ossebaard HC, Van Gemert-Pijnen JE. Persuasive System Design Does Matter: A Systematic Review of Adherence to Web-Based Interventions. J Med Internet Res. 2012;14(6):e152.

45. Donkin L, Christensen H, Naismith SL, Neal B, Hickie IB, Glozier N. A Systematic Review of the Impact of Adherence on the Effectiveness of e-Therapies. J Med Internet Res. 2011;13(3):e52.

46. Su W-C, Chih M-Y. Is More eHealth System Use Better for Cancer Patients and Family Caregivers? A Literature Review. American Medical Informatics Association Poster. 2016.

47. Kaushal N, Rhodes RE. Exercise habit formation in new gym members: a longitudinal study. Journal of Behavioral Medicine. 2015;38(4):652-63. 

Part 2

The evaluation of the implementation of e-Vita 



\section{Chapter 4}

The Added Value of Log Data Analyses of the Use of a Personal Health Record for Patients with Type 2 Diabetes Mellitus

Preliminary Results

F. Sieverink, S. Kelders, A. Braakman-Jansen, J. van Gemert-Pijnen Journal of Diabetes Science and Technology, 2014; 8(2): 247-255 DOI: $10.1177 / 1932296814525696$ 


\section{Abstract}

The electronic personal health record (PHR) is a promising technology for improving the quality of chronic disease management. Until now, evaluations of such systems have provided only little insight into why a particular outcome occurred.

The aim of this study is to gain insight into the navigation process (what functionalities are used, and in what sequence) of e-Vita, a PHR for patients with type 2 diabetes mellitus (T2DM), to increase the efficiency of the system and improve the long-term adherence. Log data of the first visits in the first 6 weeks after the release of a renewed version of e-Vita were analysed to identify the usage patterns that emerge when users explore a new application. After receiving the invitation, $28 \%$ of all registered users visited e-Vita.

In total, 70 unique usage patterns could be identified. When users visited the education service first, $93 \%$ of all users ended their session. Most users visited either 1 or 5 or more services during their first session, but the distribution of the routes was diffuse. In conclusion, log file analyses can provide valuable prompts for improving the system design of a PHR. In this way, the match between the system and its users and the long-term adherence has the potential to increase. 


\section{Introduction}

The aging population and increased prevalence of chronic care requires an integral approach to disease management that is well coordinated and consistent with (inter)national care standards in order to support a shift from institutionalized care to home care [1-3]. Disease management may be viewed as a set of interrelated services that spans the continuum from prevention and self-management to intramural care for patients with chronic diseases [4-6]. Information- and communication technology (eHealth) will play an important role in disease management, e.g. in providing online support for self-management, in improving information exchange among professionals and with patients, as well as in monitoring the performance of the disease management program [7, 8].

The electronic personal health record (PHR) is a promising technology for improving the quality of chronic disease management $[9,10]$. The Markle Foundation defined a PHR as "an electronic application through which individuals can access, manage, and share their health information and that of others for whom they are authorized, in a private, secure and confidential environment" [11]. Many researchers adopted this definition over the years [1214]. However, PHRs are becoming more complex and potential functions of current PHRs may not only include sharing clinical and personal data (e.g. history, test results, treatment, appointments), but may also include self-management support, patient-provider communication, information about illness, peer support or monitoring health behaviour data [13].

There are several potential benefits of using a PHR. Access to health data, health information and communication applications have the potential to empower patients in managing their diseases. In addition, deploying a PHR may reduce geographical and communication barriers. An ongoing connection between patient and caregiver may even lead to a transition from episodic to continuous care, which in turn has the potential to shorten the time to address disease-related complaints that may arise $[12,13]$.

Despite the potential benefits of a PHR, the use of such systems in diabetes care has only led to small improvements in diabetes quality measures that were of marginal clinical relevance [10] and up to now, evaluations have only provided little insight into why a particular outcome did occur $[15,16]$. Therefore, it is necessary to look for new methodological approaches that go beyond a before and after measurement of health outcomes, for example, by exploring the process by which users find the needed information, share information and gain benefits out of it [17]. This information is valuable in understanding how individuals want to use the system and what they are willing to do with it [12]. In other words, the logic of the content structure should match with the mental models that the users hold, in order to increase the efficiency of the system and improve the long-term adherence to the PHR. 
Log data have the potential to identify the navigation process (what functionalities are used, and in what order) on a PHR $[17,18]$. With these analyses, it is important to not only investigate the amount of use, because more exposure to a PHR will not necessarily lead to improved health outcomes and may even be an indicator for unfocused and strategic use and inefficient systems [17].

The aim of this study is to collect input for increasing the match between users and the system e-Vita, a PHR for patients with type 2 diabetes mellitus (T2DM). To understand the usage patterns that emerge when users navigate over the PHR, we conducted a log file analysis. Prior studies showed that the attrition starts when users "get lost" in the intervention $[18,19]$. Because a first impression is important, we therefore used the log files of the first visit to the PHR, to identify how users explore a new intervention. This information is important in modifying the content and the design in order to increase the efficiency of eVita and, in turn, increase the adherence of users, the chances of experiencing benefits and patient empowerment.

\section{Methods}

\section{Parent study and participants}

The analyses were performed on data collected in the parent study for effectively implementing a PHR (e-Vita) for patients with chronic diseases. In turn, this study is part of three larger studies on the effects of using a PHR in primary care for patients with T2DM, heart failure (HF) or chronic obstructive pulmonary disease (COPD). This article will focus on data collected in the T2DM-study (ClinicalTrials.gov number NCT01570140).

All participants in this study are diagnosed with T2DM and aged over 18 years. Potential participants were excluded in case of mental retardation or disorders, insufficient knowledge of the Dutch language, cognitive impairment or a short life expectancy ( $\leq 1$ year) due to terminal illnesses.

\section{Intervention}

The PHR e-Vita is an initiative of the Dutch foundation Care Within Reach, a partnership between Philips and Achmea, a Dutch health insurance company. According to Van GemertPijnen et al. [20], a web-based intervention can be seen as the whole of the content, system and the services it provides. In this view, interaction is not just content, system or service, but it rather is an integral part of an intervention. Therefore, we will describe the platform eVita according to these categories. 


\section{Content}

The content of e-Vita was created by experts in response of twelve interviews with patients with T2DM about their thoughts and feelings about living with T2DM and its treatment. Also, observations, interviews and interactive sessions were conducted to gain insight in experiences of health care professionals regarding the treatment of patients with T2DM. With this information, a PHR for patients with T2DM in primary care was developed. The main content of the PHR consists of insight in personal health data, self-monitoring health values, education and a coach for reaching personal health-related goals.

\section{System}

When logging on for the first time to e-Vita, every user sees a pop-up with a brief explanation about e-Vita and the services that can be found on the website. After the pop-up, every user was directed to the home page (Figure 1). From there, users were able to access all functionalities of the PHR.

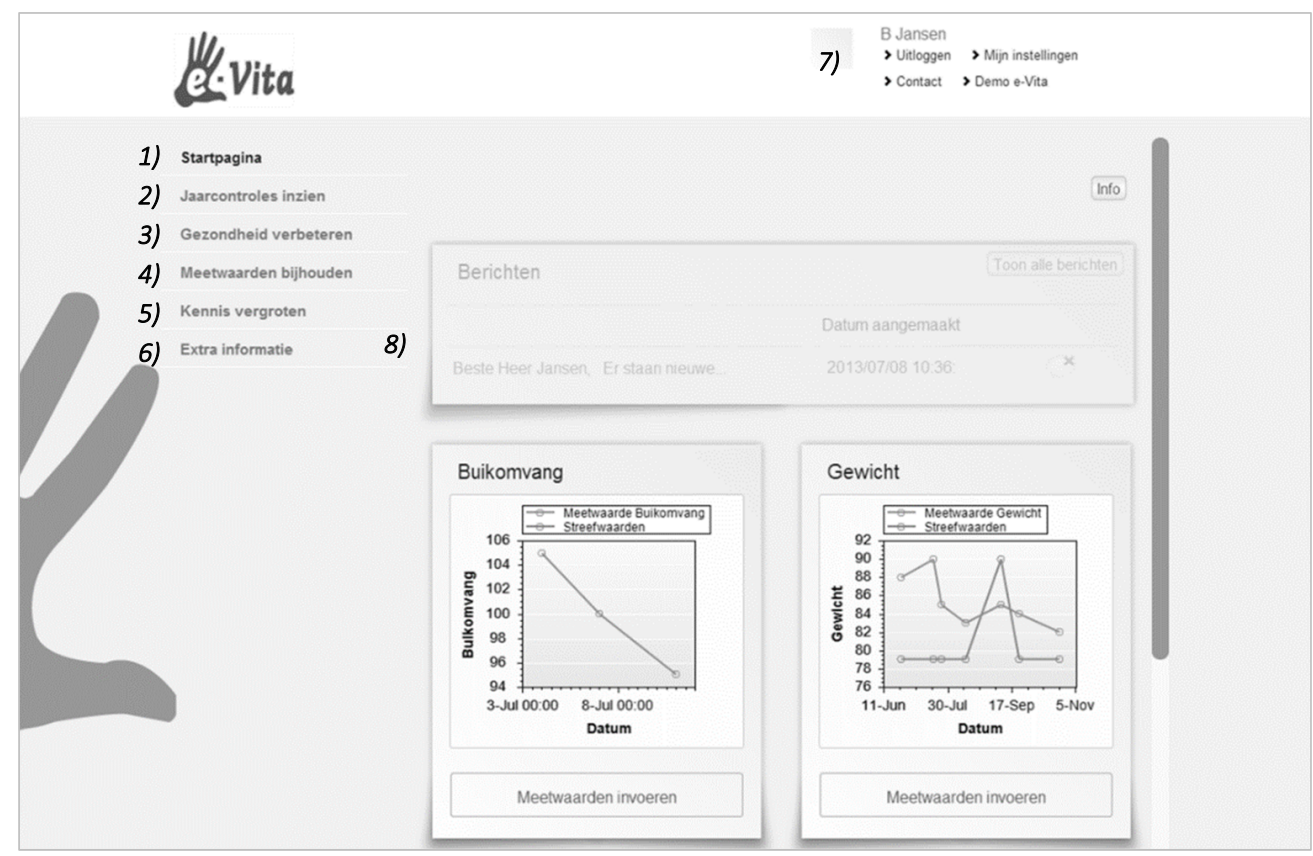

Figure 1. Screenshot of the home page of e-Vita 


\section{Service}

The system e-Vita consists of the following set of interrelated services, which can be accessed via the home page (the numbers between the brackets correspond with the numbers in Figure 1:

2) Insight in health values, provided by the general practitioner (GP). The data was updated after the annual check-up. All values are explained via an information button.

3) An online coach for guidance when working on personal, health-related goals.

4) Self-monitoring personal health values, where users can register the values they measured for blood pressure, waist circumference, weight and BMI.

5) An education module with text and movies about T2DM. Part of the offered education will be tailored to the patient. The content is provided by an independent foundation and checked by physicians.

6) Additional information about T2DM, where the patient will be directed to an external website.

7) Account settings where the user can change personal information.

8) Inbox with personal messages.

\section{Interaction}

Users' interaction with the system was only web-based. When participants finished an education module, a message was sent to the users' health care provider (in most cases, this was the users' primary care nurse), giving health care providers the opportunity to use the information as a topic of conversation during face-to-face appointments in the general practice. Users received system messages when new education or personal messages with feedback from the coach were available (8). The interaction was unidirectional and users were not able to send their own messages to their health care provider and coach (and vice versa).

\section{Data collection}

In July 2013, a renewed and extended version of e-Vita was released. All registered users were informed about this new release and were invited via e-mail to visit the PHR. Every visit to e-Vita was tracked objectively by collecting log data. In this paper, we focus on the log data of the first six weeks after the release. No major changes were made to e-Vita in this period.

The log files contained anonymous records of actions performed by each participant. For every action on e-Vita, the following information was collected by the web server and added to a log file: 1) the participants' identification number, 2) time and day of the action, 3) the type of action taken and 4) optional additional information about the action (for example, what information was viewed by the users or what personal health goals are added). For 
every user, sessions (actions taken between logging in and logging out to the system) were identified. When a user logged in to e-Vita within half an hour after the last action, this was considered to be the same session. When logging in to e-Vita, every user had to accept the general conditions which contained an informed consent for logging for research purposes. With accepting the general conditions, the user gave permission for logging their actions.

\section{Results}

\section{General}

At the time of the release of the renewed version of e-Vita, 1197 potential participants were invited to register on the PHR. In total, 568 users (46\%) agreed to register. After the invitation via e-mail to visit the renewed e-Vita, 161 users visited the platform at least once in the first six weeks ( $28 \%$ of the registered users and $13 \%$ of the potential participants).

In total, 249 sessions were conducted, an average of 1.5 sessions per visitor in this period. In Figure 2, an overview of the distribution of these sessions over the weeks is given.

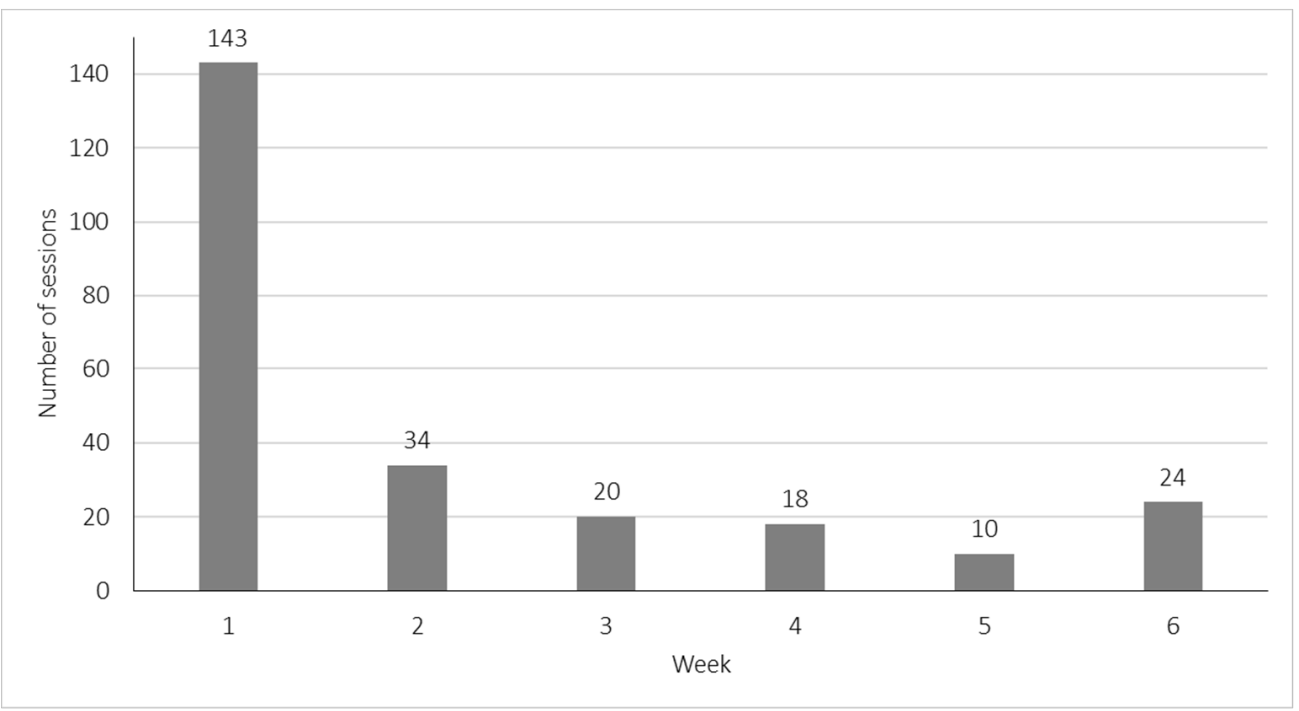

Figure 2. Number of sessions per week in the first six weeks after the release of the renewed e-Vita In the first week after the release, 110 different users visited e-Vita in 143 sessions. In the following weeks, the number of sessions decreased. Overall, most users visited e-Vita on Tuesday or Wednesday in the afternoon. 


\section{The first session}

An overview of all usage patterns that were identified for the first visit, is given in Appendix 1. In total, 70 different usage patterns were identified. An overview of the services that were visited as a first step after the login, is given in Table 1.

Table 1. Services that were visited as a first step after the login $(N=161)$

\begin{tabular}{|l|c|}
\hline Service & $\mathrm{n}(\%)$ \\
\hline Health values & $55(34 \%)$ \\
\hline Education & $46(29 \%)$ \\
\hline Inbox & $21(13 \%)$ \\
\hline End of session & $17(11 \%)$ \\
\hline Coaching & $10(6 \%)$ \\
\hline Settings & $6(4 \%)$ \\
\hline Self-monitoring & $5(3 \%)$ \\
\hline Information & $1(1 \%)$ \\
\hline
\end{tabular}

Regarding the first step after the login, three main usage patterns were identified. First, of the 161 users, 55 (34\%) visited the service for insight in health values directly after the first login. After this step, the user was most likely to follow the structure of the main menu (marked with the numbers 2 to 6 in Figure 1). This route occurred nine times.

Second, when a user visited the education service as the first step after the login, 93\% (42 out of 46 users) ended their session there. In total, 36 of 42 users (86\%) viewed less than five education topics, while $14 \%$ viewed five or more topics (median is 1 topic).

Third, 17 users ended their session immediately after the first login. Seven users returned for a second visit of the platform in the first six weeks after the release. During the second session, six of these users visited the service for insight in health values first. The distribution of the other usage patterns was diffuse. In Table 2, an overview is given of the number of services that are visited during the first session. 
Table 2. Number of visited services during the first login, before ending the session

\begin{tabular}{|l|c|}
\hline Number of visited services & $\mathrm{n}(\%)$ \\
\hline Login - end of session & $17(11 \%)$ \\
\hline One service & $60(37 \%)$ \\
\hline Two services & $19(12 \%)$ \\
\hline Three services & $11(7 \%)$ \\
\hline Four services & $12(7 \%)$ \\
\hline Five or more services & $41(25 \%)$ \\
\hline
\end{tabular}

In their first session, 60 users (37\%) visited one service after the login. A quarter of all users visited five or more services after the first login. The percentages of users that visited two to four services, is lower.

\section{Discussion}

\section{Principal results}

The aim of this study was to collect input for increasing the match between users and e-Vita, a PHR for patients with T2DM in primary care, in order to increase the adherence of users, the chances of experiencing benefits and patient empowerment. Therefore, we conducted a log file analysis to gain insight in the usage patterns that emerge when users explore the PHR.

After receiving an invitation to visit the renewed version of e-Vita, only $28 \%$ of the registered users visited the PHR at least once in the first six weeks. The number of logins decreased over the weeks, which is a common finding in eHealth research, also known as the law of attrition [21].

In terms of the usage patterns that emerged, there are some important findings. First, users were most likely to follow the structure of the main menu. The results of our analyses have thus shown that the layout of the menu structure is important, and that the routes that users take on a PHR probably can be influenced by the sequence in which the services are presented. This information is valuable in marking the intended routes by the developer on a PHR.

Second, when users visit the education service as the first step after the first login, 93\% ended their session. When users visit the education service after they visited another service, this pattern was less likely to emerge. There are several possible explanations for this finding. The first explanation might be that the amount of information that is presented in the education service is too overwhelming, causing users to end their session. A second explanation is that the users who visited the education service as the first step after the first login, spend more time to explore the available topics and explore the rest of the PHR in the next sessions. 
Results have shown that de median number of visited education topics is one, supporting the idea that viewing the education service as a first step after the first login, might be too overwhelming. This information can be used to improve the design of the service, for instance by making the design more clear and compact.

Third, except the route that follows the menu structure on the home page of the PHR, the distribution of the other routes was very diffuse. This is an indication that it might be unclear for users how they should explore the PHR, and might possibly hinder a second visit. Because diffuse patterns may be an indication for unfocused and non-strategic use [17], it might therefore be useful for developers to give an explanation to users about the possibilities of a PHR and to guide users over the platform.

Last, when a user logged in for the first time, they were either likely to log out after visiting one service, or to visit five or more services on the website, indicating that the first impression of the PHR of users could be more attractive. However, when users overcame this first impression, they made an effort to explore the rest of the PHR. This is a prompt for more persuasive support at the first login, for guiding users over the PHR.

In summary, our results of the log file analyses have shown that the identification of usage patterns can provide us valuable information about how users navigate over a PHR when visiting it for the first time, which is in line with previous research [17, 18]. Also, the importance of the layout has been demonstrated. This information can be used to make the purpose of e-Vita more evident with the first login. For example, a tutorial could be made to show new users the evident and effective routes.

\section{Limitations}

The first limitation of this study is that we did not involve the users in analysing the log data. In other words, we have not checked our interpretations regarding the usage patterns that emerged and we are not able to derive mental models out of the results. It is therefore important to involve users in the future, to learn more about the mental models the users hold when using an PHR, like Tang previously suggested [12]. On the other hand, we were interested in usage patterns in this study and the log data has provided us with objectively measured and real-time information that would have been hard to be recalled by users after their first session on the PHR.

Second, this data have only revealed the usage in the first six weeks after a new release in a relative small sample, and we were not able yet to track the usage over a longer period of time to see what long-term usage patterns emerge. 


\section{Future research}

The results of the analyses have raised several questions for our future research. First, it would be interesting to track the usage of e-Vita over a longer period of time, e.g. over three to six months. In this way, we are able to track changes in usage patterns over time and more definitive usage patterns can be revealed.

Second, it would be interesting to link these patterns to the information about the users, for example demographics (age, educational level, disease history), health values, opinions about the PHR or the quality of care, to predict what factors influence a return to the PHR, and, in addition, to identify the most effective patterns in terms of the adherence to the PHR, satisfaction about the care that has been delivered and the development of selfmanagement skills as a result of using the PHR. Third, it would be useful to conduct an interview study concerning the mental models that the users hold when navigating over a PHR.

\section{Conclusions}

In conclusion, we have shown that log file analyses can provide valuable prompts for improving the system design of eHealth applications, for example a PHR in order to increase both the adherence to and the efficiency of eHealth applications. 


\section{References}

1. Shaw, J.E., R.A. Sicree, and P.Z. Zimmet, Global estimates of the prevalence of diabetes for 2010 and 2030. Diabetes Research and Clinical Practice, 2010. 87(1): p. 4-14.

2. Klonoff, D.C., Using telemedicine to improve outcomes in diabetes-an emerging technology. Journal of diabetes science and technology (Online), 2009. 3(4): p. 624.

3. Bellazzi, R., Telemedicine and diabetes management: current challenges and future research directions. Journal of diabetes science and technology (Online), 2008. 2(1): p. 98.

4. Bodenheimer, T., et al., Patient self-management of chronic disease in primary care. JAMA, 2002. 288(19): p. 2469-2475.

5. Norris, S.L., et al., Recommendations for healthcare system and self-management education interventions to reduce morbidity and mortality from diabetes. American Journal of Preventive Medicine, 2002. 22(4 SUPPL. 1): p. 10-14.

6. Wagner, E.H., et al., Quality improvement in chronic illness care: a collaborative approach. The Joint Commission journal on quality improvement, 2001. 27(2): p. 63-80.

7. Verhoeven, F., et al., Asynchronous and Synchronous Teleconsultation for Diabetes Care: A Systematic Literature Review. Journal of Diabetes Science and Technology, 2010. 4(3): p. 666-684.

8. Goldberg, H.I., et al., Using an Internet comanagement module to improve the quality of chronic disease care. Joint Commission Journal on Quality and Patient Safety, 2003. 29(9): p. 443-451.

9. Osborn, C.Y., et al., Patient web portals to improve diabetes outcomes: A systematic review. Current Diabetes Reports, 2010. 10(6): p. 422-435.

10. Tenforde, M., et al., The Association Between Personal Health Record Use and Diabetes Quality Measures. Journal of General Internal Medicine, 2012. 27(4): p. 420-424.

11. Markle Connecting for Health. The Personal Health Working Group final report. July 1, 2003 Retrieved from: https://www.markle.org/publications/1429-personal-health-working-group-finalreport.

12. Tang, P.C., et al., Personal Health Records: Definitions, Benefits, and Strategies for Overcoming Barriers to Adoption. Journal of the American Medical Informatics Association, 2006. 13(2): p. 121126.

13. Pagliari, C., D. Detmer, and P. Singleton, Potential of electronic personal health records. BMJ, 2007. 335(7615): p. 330-333.

14. Tenforde, M., A. Jain, and J. Hickner, The value of personal health records for chronic disease management: what do we know? Family Medicine-Kansas City, 2011. 43(5): p. 351.

15. Black, A.D., et al., The Impact of eHealth on the Quality and Safety of Health Care: A Systematic Overview. PLoS Med, 2011. 8(1): p. e1000387.

16. Kaplan, B., Evaluating informatics applications-some alternative approaches: theory, social interactionism, and call for methodological pluralism. International journal of medical informatics, 2001. 64(1): p. 39-56.

17. Han, J.Y., Transaction logfile analysis in health communication research: Challenges and opportunities. Patient Education and Counseling, 2011. 82(3): p. 307-312.

18. Kelders, S.M., E.T. Bohlmeijer, and J.E.W.C. Van Gemert-Pijnen, Participants, usage, and use patterns of a web-based intervention for the prevention of depression within a randomized controlled trial. Journal of Medical Internet Research, 2013. 15(8). 
19. Nijland, N., et al., Factors influencing the use of a Web-based application for supporting the selfcare of patients with type 2 diabetes: a longitudinal study. Journal of Medical Internet Research, 2011. 13(3).

20. Van Gemert-Pijnen, J.E.W.C., et al., A holistic framework to improve the uptake and impact of eHealth technologies. Journal of medical Internet research, 2011. 13(4).

21. Eysenbach, G., The law of attrition. Journal of Medical Internet Research, 2005. 7(1). 


\section{Appendix 1 - Usage patterns identified for the first session after the release.}

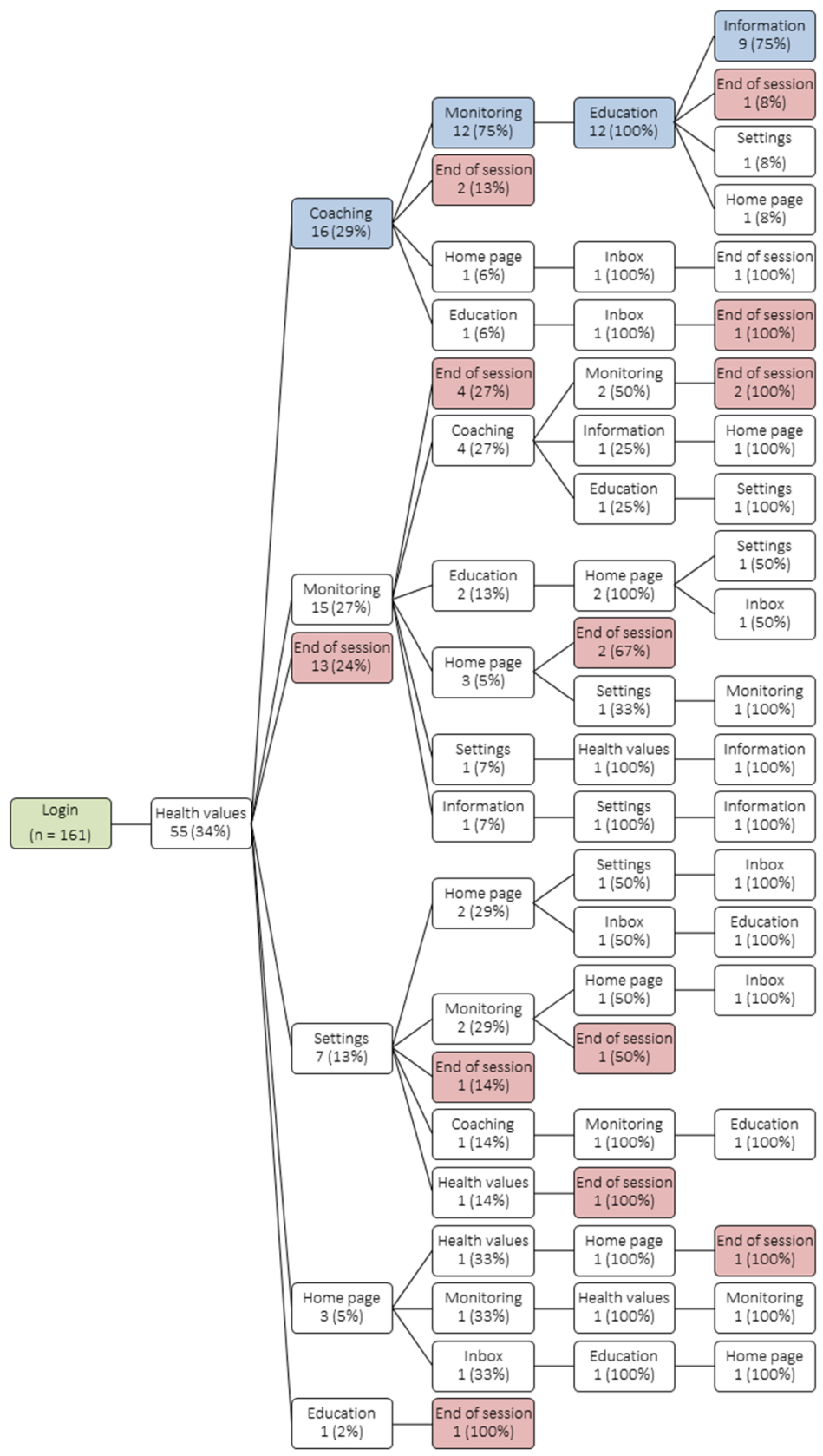




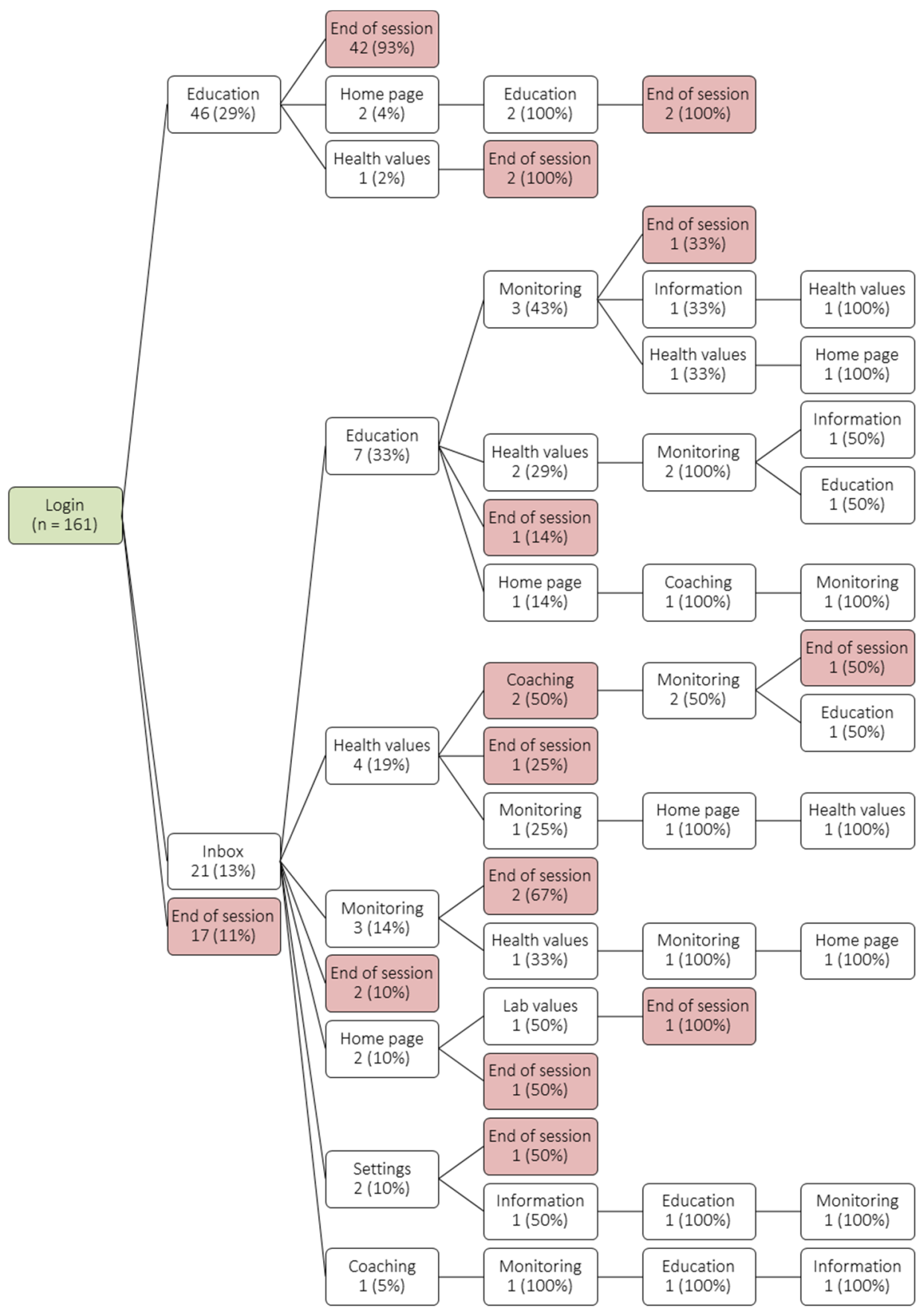




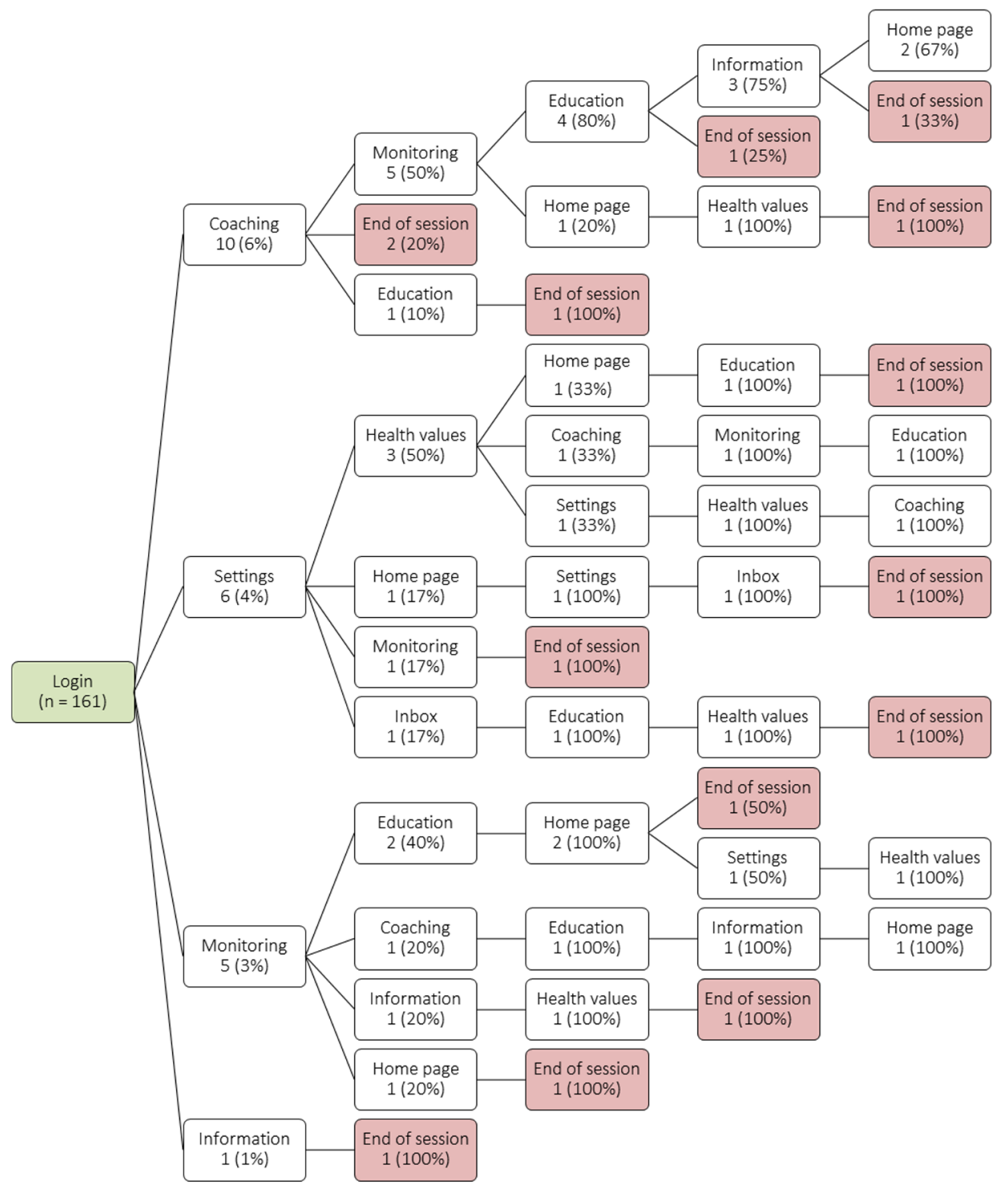






\section{Chapter 5}

\section{The Diffusion of a Personal Health Record for Patients with Type 2 Diabetes Mellitus in Primary Care}

F. Sieverink, A. Braakman-Jansen, Y. Roelofsen, S. Hendriks, R. Sanderman, H. Bilo, J. van Gemert-Pijnen International Journal on Advances in Life Sciences, 2014; 6(3\&4): 177-183 


\section{Abstract}

A Personal Health Record is a promising technology for improving the quality of chronic disease management. Despite the efforts that have been made in a research project to develop a Personal Health Record for patients with type 2 diabetes mellitus in primary care (e-Vita), considerable differences have been reported between the number of registered users in the participating primary practices. Interviews among practice nurses indicated that a lack of infrastructure (integration in daily care processes), the ability to try the Personal Health Record with minimal investments and without commitment (trialability) and the relative advantage of using a Personal Health Record in comparison with other methods were important factors for the diffusion of the Personal Health Record in primary care.

Keywords - Personal Health Record; type 2 diabetes mellitus; implementation; diffusion of innovation; interviews; contextual inquiry; value specification; summative evaluation. 


\section{Introduction}

In this paper, we provide an extended version of our paper as presented at eTelemed 2014, the sixth International Conference on eHealth, Telemedicine and Social Medicine [1]. We present the results of an interview study to identify factors perceived as important in the diffusion of a personal health record (PHR) for patients with type 2 diabetes mellitus (T2DM) in primary care, from the perspective of health care workers.

\section{Personal Health Records}

The aging population and the increasing need for chronic care requires an integral approach to disease management that is well coordinated and consistent with (inter)national care standards in order to support a shift from institutionalized care to home care [2-4]. Disease management may be viewed as a set of interrelated services that spans from prevention and self-management to intramural care for patients with chronic diseases [5-7]. Informationand communication technology (eHealth) will play an important role in disease management, e.g., in providing online support for self-management, in improving information exchange among professionals and with patients, as well as in monitoring the performance of the disease management program $[8,9]$.

The electronic PHR is a promising technology for improving the quality of chronic disease management $[10,11]$. A PHR can be defined as "an electronic application through which individuals can access, manage, and share their health information and that of others for whom they are authorized, in a private, secure and confidential environment" [12], a definition that is adopted by many researchers over the years (e.g., [13-15]. However, PHRs are becoming more complex and potential functions of current PHRs may not only include sharing clinical and personal data (e.g., history, test results, treatment, appointments), but may also include self-management support (patient-provider communication, information about the illness, peer support or monitoring health behavior data) [14]. Potential benefits of a PHR include empowering patients in managing their diseases and the reduction of geographical and communication barriers. This may, in turn, lead to a transition from episodic to continuous care, which has the potential to shorten the time to address diseaserelated complaints that may arise $[13,14]$.

Despite these benefits, the use of such systems in diabetes care has only led to small improvements in diabetes quality measures that were of marginal clinical relevance [10], and up to now, evaluations have only provided little insight into why a particular outcome did occur $[16,17]$. Consequently, the added value of the existing evidence is often limited for decision making in relation to the strategic direction of implementation efforts [18]. To gain insight into factors that contribute to a successful implementation of eHealth technologies 
in daily health care processes, it is therefore necessary to look for methodological approaches that go beyond a baseline and follow-up measurement of health outcomes.

\section{e-Vita}

The PHR e-Vita is an initiative of the Dutch foundation Care Within Reach (in Dutch: Zorg Binnen Bereik), a partnership between Philips and Achmea, a Dutch health insurance company. Currently, e-Vita is deployed in in the Netherlands via three trials to study the effects of the PHR for patients with T2DM, chronic heart failure or COPD. In this paper, we will focus on results from the T2DM study. For patients with T2DM, the main content of eVita consists of insight into personal health data (e.g., lab values, blood pressure), selfmonitoring health values (e.g., weight), education and a coach for reaching personal healthrelated goals.

The T2DM research project consists of two parts. First, a prospective observational cohort study (a benchmarking study) is being conducted to assess clinical parameters and, on the long term, quality of life and disease-related complications. Within this study, questionnaires among participants are administered periodically and health data and blood samples are collected. When patients agreed to participate in this benchmarking study, they are invited to participate in the PHR trial. Main goal of this trial is to study the effects of using a PHR in primary care for patients with T2DM (ClinicalTrials.gov number NCT01570140) [19]. In total, 44 primary care practices participate in this trial.

\section{Diffusion of Innovations}

Despite the efforts that have been made to develop a technology that has added value in the treatment of patients with T2DM in primary care, differences in the diffusion of the PHR are signaled between the primary practices that participate in the research project. The pace at which new innovations in health care diffuse through the system, depends on several factors. In Table 1, an overview of critical factors for the diffusion of innovations according to Cain \& Mittman [20] is given. To gain insight into the factors that influence the diffusion of a PHR in a primary care practices, an evaluation via interviews has been conducted. The main research question was:

What factors influenced the diffusion of a PHR for patients with type 2 diabetes (T2DM) in primary health care, according to primary health care workers? 
The sub-questions were:

1. What were the reasons and incentives of primary health care workers to participate in research project regarding the use of a PHR?

2. What training did the primary health care workers receive at the start of the research project?

3. How did the primary health care workers embed the PHR in their daily care routines?

4. What were the perceived and expected barriers and facilitators for embedding a PHR in daily care routines, according to primary health care workers?

5. What are the expectations of primary health care workers regarding the use of PHRs in the future?

The outcome of the interviews provides critical factors for the improvement of the diffusion and implementation process of a PHR in primary care.

This paper is organized as follows: in Section II, we will describe how the interviews were conducted. In Section III, the results of the interviews are described. In Section IV, the results and directions for future research are discussed. Finally, in Section $V$, the conclusions of this paper are given. 
Table 1. Critical dynamics for the diffusion of innovations [20]

\begin{tabular}{|c|c|}
\hline Critical Dynamic & Explanation \\
\hline $\begin{array}{l}\text { Relative } \\
\text { advantage }\end{array}$ & $\begin{array}{l}\text { The higher the potential of the technology in comparison to current } \\
\text { practice, the more rapidly it will diffuse. }\end{array}$ \\
\hline Trialability & $\begin{array}{l}\text { The ability to try out an innovation without total commitment and } \\
\text { with minimal investment. }\end{array}$ \\
\hline Observability & $\begin{array}{l}\text { The extent to which potential users follow the adoption of an } \\
\text { innovation by others. }\end{array}$ \\
\hline $\begin{array}{l}\text { Communication } \\
\text { channels }\end{array}$ & $\begin{array}{l}\text { The communication channels through which others communicate } \\
\text { about the innovation. }\end{array}$ \\
\hline $\begin{array}{l}\text { Homophilous } \\
\text { groups }\end{array}$ & Innovations diffuse faster among groups with similar characteristics. \\
\hline $\begin{array}{l}\text { Pace of } \\
\text { innovation }\end{array}$ & $\begin{array}{l}\text { The extent to which innovations evolve and are being altered by its } \\
\text { users. }\end{array}$ \\
\hline $\begin{array}{l}\text { Norms, roles and } \\
\text { social networks }\end{array}$ & $\begin{array}{l}\text { Innovations are shaped by the rules, formal hierarchies, and informal } \\
\text { mechanisms of communication operative in the social systems in } \\
\text { which they diffuse. }\end{array}$ \\
\hline Opinion leaders & $\begin{array}{l}\text { Individuals whose opinions are respected by others in a population } \\
\text { affect the pace of diffusion. }\end{array}$ \\
\hline Compatibility & $\begin{array}{l}\text { The ability of an innovation to coexist with technology and social } \\
\text { patterns already in place. }\end{array}$ \\
\hline Infrastructure & $\begin{array}{l}\text { The presence of some form of infrastructure that cluster with the } \\
\text { innovation. }\end{array}$ \\
\hline
\end{tabular}

\section{Methods}

In this section, we will present the framework for the interview study, the participants, the design and procedure of the interviews and how the data was analysed.

\section{The Center for eHealth Research (CeHRes) Roadmap}

The CeHRes roadmap [21] is a framework that is used to develop new and to evaluate and improve existing eHealth technologies. The roadmap states that eHealth development is a participatory process and that development is intertwined with implementation into daily health care processes. The roadmap consists of five different phases (Figure 1): 
1. Contextual inquiry: First, the needs and problems of the stakeholders (e.g., patients, caregivers, health insurers) are described, in order to gain insight into the context and whether or how technology can contribute to minimizing problems.

2. Value specification: Second, information about the added values that keystakeholders attribute to the eHealth intervention is gathered. Together with the contextual inquiry, the value specification provides the functional requirements for the design of the technology.

3. Design: On the basis of these requirements, prototypes of the technology are developed and tested.

4. Operationalization: The final version of the eHealth technology is launched.

5. Summative evaluation: Finally, the uptake, effects and impact of the eHealth intervention are evaluated.

According to the roadmap, the development of eHealth technology also requires continuous evaluation cycles after every step, in order to create eHealth technologies that have the potential to diffuse among its end-users.

For this study, the interviews serve as both a forward evaluation (contextual inquiry and value specification) and a backward (summative) evaluation to gain insight into the uptake and impact of e-Vita as well as into the possibilities to improve the content of e-Vita according to health care providers.

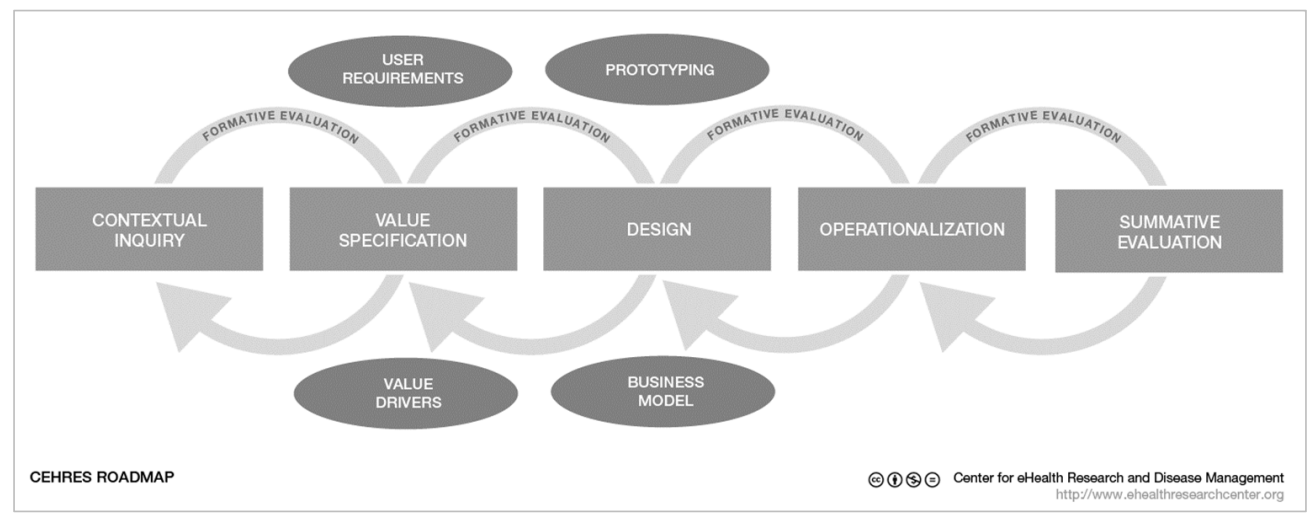

Figure 1. The Center for eHealth Research and Disease Management Roadmap [21]

\section{Participants}

For the interview study, primary care nurses (PNs) of general practices in Drenthe, a province in the north of the Netherlands, were invited to participate in the interview study. In the Netherlands, PNs are the main responsible caregivers for educating patients about their (chronic) disease, advising patients regarding medication use and lifestyle changes and 
performing health checks. In the current trial, all selected PNs are responsible for explaining the purpose of the PHR to the participants in the study and administering questionnaires regarding the effects of the PHR. No guidelines for intended use of the PHR are defined.

To reveal the differences between the diffusion processes of practices with high and low numbers of participants, potential practices for the interview study were selected by the means of an inclusion percentage (high, middle, low). The inclusion percentage was calculated as follows:

\section{Inclusion percentage $=$}

(number of included patients for the benchmarking study / total number of patients with T2DM in the practice)*100.

Our aim was to conduct five interviews in every group, 15 interviews in total. We therefore invited the five primary practices with the highest and lowest percentages. Also, five primary practices with an average inclusion percentage were invited to participate. When primary practices had indicated on beforehand that the inclusion of participants was postponed due to explainable circumstances (e.g., long-term diseases among the staff), practices were not contacted to participate in the interview study.

All PNs who met the criteria for the interview study received an e-mail with information about the purpose and the topics of the interview. When PNs agreed to participate, they were contacted to make an appointment for the interview.

\section{Design and Procedure}

Semi-structured in-depth interviews were conducted. During the interviews, questions were asked regarding the following themes: reasons and incentives to use and implement a PHR in their primary practice, the use and users of the PHR so far, bottlenecks and barriers that are encountered or expected, the (positive) results so far and the expected changes a PHR will make in the primary health care for patients with T2DM. The duration of the interviews was 45-60 minutes (non-stop). All participating PNs received a gift voucher of 50 euros. Ethical approval for this interview study was obtained by the ethics committee of the University of Twente.

\section{Data Analysis}

All interviews were transcribed verbatim and themes and categories were subsequently coded via open coding, axial coding and selective coding [22]. In this way, recurring themes and items of interest regarding the implementation and use of eHealth technologies in primary health care practice were identified. Occurring themes were categorized using the critical dynamics for the diffusion of innovations, according to Cain \& Mittman [20]. 


\section{Results}

In this section, we will present the results of the interview study.

\section{General Results}

After receiving the invitation, 11 PNs agreed to participate in the interview study. An overview of the number of potential and included participants for the primary care practice of every PN at the start of the interviews (August 2013) is provided in Table 2.

Table 2. Overview of participating primary practices in the interview study

\begin{tabular}{|c|c|c|c|}
\hline \multirow{2}{*}{$\begin{array}{l}\text { PN } \\
\text { number }\end{array}$} & \multirow{2}{*}{$\begin{array}{l}\text { Inclusion } \\
\text { group }\end{array}$} & \multicolumn{2}{|l|}{ Patients } \\
\hline & & $\begin{array}{l}\text { \# T2DM } \\
\text { patients }\end{array}$ & $\begin{array}{c}\text { \# participants } \\
\text { included (\%) }\end{array}$ \\
\hline 1 & High & 204 & $126(62)$ \\
\hline 2 & High & 56 & 33 (59) \\
\hline 3 & High & 61 & $37(61)$ \\
\hline 4 & High & 88 & $45(51)$ \\
\hline 5 & High & 146 & $63(66)$ \\
\hline 6 & Middle & 98 & $22(22)$ \\
\hline 7 & Middle & 122 & $25(20)$ \\
\hline 8 & Middle & 182 & $45(25)$ \\
\hline 9 & Middle & 94 & 18 (19) \\
\hline 10 & Low & 163 & $4(2)$ \\
\hline 11 & Low & 235 & $7(3)$ \\
\hline
\end{tabular}

In total, 5 PNs of practices with high inclusion percentages, 4 PNs of practices with average inclusion percentages and 2 PNs of practices with low inclusion percentages participated in the interview study. 


\section{Reasons and Incentives to Participate}

In total, 5 PNs (interview numbers 2, 4, 7, 8 and 11) indicated that they participate in the trial because they find it important to stimulate the development of self-management skills of their patients:

\section{"If we can offer patients tools to learn about their own disease and to take their own responsibilities, we must not miss this opportunity."}

Two PNs indicated that they want to keep abreast of the times (interview numbers 1 and 2), that they are curious about the added value of a PHR in primary practice (interview numbers 1 and 6 ), and that they want to offer their patients something extra during their treatment, for example information about T2DM (interview numbers 7 and 8). Also, one PN indicated that the primary care practice wanted to participate because of their (relatively young) patient population (interview number 3 ) or to participate in a research project besides the daily working routines (interview number 9). Two PNs (interview numbers 6 and 11 ) indicated that they were curious about the results of the benchmarking study: how do their practices perform in comparison with other practices and how satisfied are their patients about the care they receive?

\section{Training}

Regarding the training the PNs received before the start of the project, 6 PNs indicated that they attended a plenary information meeting (interview numbers 1, 2, 3, 4, 8 and 9). During this meeting, information was given about the purpose and the course of the research project and instructions were given regarding the inclusion of participants and administering the questionnaires for the benchmarking study. One PN (interview number 4 ) indicated that there was a short explanation about the purpose and the functions of the PHR during that meeting.

Eight PNs indicated that they were not trained in using the PHR and how to integrate the PHR in daily care routines (interview numbers $1,3,5,7,8,9,10,11$ ). One PN received some instructions for using the PHR (interview number 6 ) and another PN logged in once with one of the researchers (interview number 2).

\section{Integrating the PHR in Daily Care Routines}

In total, 8 PNs indicated that they did not integrate the PHR with consultations with their patients. The remaining 3 PNs occasionally ask their patients if they visited the PHR and if they have questions regarding the information on the PHR. 


\section{Perceived Barriers for Using a PHR}

All PNs indicated that there is a lack of time to use the PHR in the treatment of their patients. According to 7 PNs (interview numbers 1, 2, 3, 5, 6, 7 and 8), this is due to a lack of integration of the PHR in daily work routines:

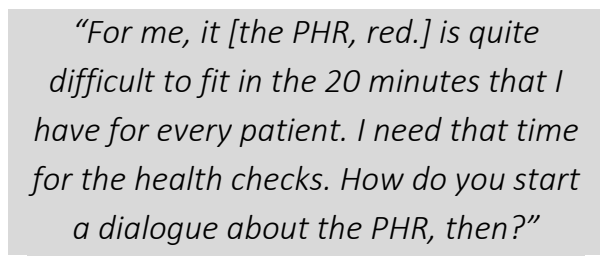

Also, 9 PNs (interview numbers 1, 2, 3, 5, 6, 7, 9, 10 and 11) indicated that they did not have access to the PHR. They were not able to see what patients see, and find it therefore difficult to promote and explain the PHR among their patients:

"It is quite difficult. You don't know

e-Vita, and you have to explain it to

the patients. That doesn't work."
"I was not able to see what patients see for a long time. I have to recommend something I don't know."

When they were able to log in to the PHR via their patients, 4 PNs (interview numbers 1, 5, 6 and 9) experienced usability problems:

"When you want to visit e-Vita, you

have to take the hurdle of logging in

$$
\text { first." }
$$

Also, 4 PNs (interview numbers 2, 8, 9 and 11) indicated that they have easy accessible alternatives:

"One patient was quite motivated, so I

printed the health data and gave it to

him. That is a much shorter way."

Regarding their patients, 5 PNs indicated that many of their patients do not have Internet access (interview numbers 1, 6, 7, 8 and 10) or experience usability problems (interview numbers 1, 2, 3, 4 and 6).

Furthermore, according to 5 PNs, the PHR does not meet the needs of their patients (interview numbers 1, 4, 5, 6 and 7), and patients are afraid that the usual care will become less personal (interview numbers 1, 6, 8 and 10). 


\section{Perceived Facilitators for Using a PHR}

Although the PHR is not yet used in daily care routines, some potential factors for the successful implementation of a PHR were mentioned during the interviews.

All PNs indicated that they could easily contact a help desk when they experienced technical problems with the PHR or when they have questions regarding the benchmarking study:

"Nothing is too much. It is really important that it is never inconvenient to have contact."

\section{"A strong point is that they are easy} to reach. When I have a problem, I send an email and the next day, I have an answer."

Second, 5 PNs (interview numbers 1, 3, 4, 7 and 10) indicated that they were able to fit the activities necessary for the benchmarking study into their own workflow:

"I made a Word file with a list of participants, this gives me an overview of the procedure."
"Every time I realize that I have to establish a system to create a routine."

Third, 4 PNs (interview numbers 4, 5, 7 and 9) indicated that triggers for using the PHR are probably important, for example, via news letters or via (financial) incentives:

"I think you should give a financial incentive, for example, a bonus from the health insurance company."
"Maybe a newsletter, a stimulus to let patients think, 'let's visit e-Vita again'."

\section{The Future of PHRs in Primary Care}

Finally, PNs were asked about their opinion regarding the future of PHRs in primary health care. In total, 5 PNs (interview numbers 1, 2, 4, 5 and 11) indicated that they find it hard to predict whether there is a future for PHRs in health care, and how PHRs will be used in the future.

On the other hand, 5 PNs (interview numbers 2, 4, 6, 9 and 11) expect better-informed patients during consultations, but they also believe that their own role is not likely to change.

Three PNs (interview numbers 3, 5 and 6) believe that a PHR in primary care will mainly be used for communication purposes between patients and health care providers. 


\section{Discussion}

The interviews indicated that, despite the participants' enthusiasm and understanding of the importance of stimulating self-management skills of patients with chronic conditions, the diffusion of the PHR for patients with T2DM in primary care is still rather low. The goal of this study was to identify the factors that influenced the diffusion. We signaled differences in the inclusion percentages between the participating primary practices in the research project, and therefore we wanted to gain insight into the factors that influenced the diffusion from the view of primary health care workers.

Because we believe that the development of eHealth technologies is an ongoing process that requires continuous evaluation cycles [21], we conducted both a forward and a backward evaluation. With this evaluation, we tried not only to gain insight into the factors that influenced the uptake and impact of a PHR, but also to identify possibilities for improving the PHR in the future.

Although we aimed to identify differences between the factors that influenced the diffusion of the PHR experienced by PNs from primary practices with high, medium and low inclusion percentages, the experiences of all PNs were fairly similar, which indicates that a high inclusion of participants in the study does not necessarily lead to using the system in daily practice. This finding made it difficult to identify factors that contributed to the use of the PHR. However, we did find some important factors that influenced the diffusion of the PHR.

First, a lack of infrastructure that is necessary for the implementation of an innovation in health care $[20,23]$ played an important role. Most PNs indicated that at the start of the research project, little attention has gone towards education and guidance regarding the integration of the PHR with daily practice, and thus, with national guidelines for the treatment of chronic diseases in primary care. Most PNs indicated that they were mainly trained to administer the questionnaires for the benchmarking study, and during that training, only little attention has gone to the content and the functions of the PHR and the integration of the system with daily practice.

As a result, the awareness of PNs regarding the added value of the PHR is low, which can reduce its diffusion and subsequent use [24]. PNs indicated that they do not use the PHR in the treatment of their patients. They have a certain amount of time for every patient during the consultations, and in this time, PNs have to finish the health check-ups (blood pressure, control of the feet, et cetera), talk with the patient about how they are doing and administer the questionnaires for the benchmarking study. Because no guidelines were given regarding the use of the PHR, asking patients about their experiences is not on top of the minds of the PNs. Also, PNs indicated that there is often no time to ask their patients about the experiences and the use of the PHR. 
A first study of log data of the PHR showed that the use by patients is suboptimal. After an invitation to visit the renewed PHR, 28\% of all registered users visited the PHR at least once, with a mean of 1.5 visits in the first six weeks [25]. It is well possible that creating a routine in using a PHR during consultations will lead to an increased use of the PHR by patients at home. After all, it is likely that patients will use a system that is being promoted by their health care providers, who are often in a relationship of trust with their patients and are therefore seen as opinion leaders [20].

An another finding of the interview study is that the trialability [20] of the PHR played an important role in the diffusion of the technology. Due to technical problems, PNs were not able to log on to the PHR with test accounts and could therefore not see what their patients see. Subsequently, PNs reported that they find it difficult to use and promote a technology they hardly know.

At the time of the interviews, test accounts were available for PNs, which enhanced the trialability of the system [20]. However, because of the reported work pressure and established working routines for administering the questionnaires regarding the benchmarking study, it often had no priority anymore to visit the PHR.

Also, 4 PNs indicated that they have easy accessible alternative tools and resources available for the PHR, for example by providing patients with lab values on paper, instead of viewing them on the PHR. In other words, the relative advantage $[20,23]$ of the PHR is rather low, which is probably another important factor for the slow diffusion of the PHR [23].

To increase the diffusion of the PHR in the future, it is therefore useful to guide health care providers in integrating the system in daily routines. By creating an infrastructure for the use of the PHR, new working routines are being established. Also, it might be of added value to appoint ambassadors: health care providers who already successfully use the PHR in daily practice. By increasing the observability of the PHR, other health care providers are able to see how others use the PHR and can acknowledge that the use of the PHR is safe and beneficial [20].

However, the results also showed that the PHR is mostly illness-driven instead of usercentred (with little attention for the needs of the end-users), indicating that involving the end-users (via a contextual inquiry and value specification [21]) is valuable in the development of new technologies [24]. By involving the end-users and having an eye for their needs, the added value of the new technology is already evident in the first stages of the developmental cycle [26], which may in turn lead to a better diffusion of the technology.

The interviews not only served as a backward evaluation, but also as an forward evaluation to gain insight into the possibilities to improve the PHR in the future. However, because of the lack of insight into the system, PNs found it rather hard to provide directions for the 
improvement of the PHR. Also, they find it hard to indicate how PHRs will be used in the future. In general, PNs expect better informed patients during consultations, but they also believe that their own role in the treatment of patients is not likely to change.

In the future, we are planning to conduct a further process analysis of the implementation of the PHR using a mixed methods approach via interviews and usability tests among both patients and health care providers. Also, we are planning to conduct advanced log file analyses, containing real-time data about the actual use of the PHR, collected by the web server. With this data, we will analyse who the actual users of the PHR are, how they use the system and how the PHR supports the users in reaching their health related goals in order to gain insight in how a PHR can be of added value in primary care.

\section{Conclusion and future work}

The results of the interviews indicate that PNs understand the importance of stimulating selfmanagement skills of patients with chronic diseases via a PHR. However, the diffusion of the PHR is still rather low, mostly due to a lack of training in using the PHR and a lack of guidance in integrating the system in daily care routines. Also, the trialability and the relative advantage of the PHR played an important role in the uptake and impact of the new system.

In the future, we will involve end-users (both patients and health care providers) in our research, in order to create a PHR that is of added value for patients with chronic diseases in primary care. 


\section{References}

1. Sieverink, F., et al. The Uptake and Impact of a Personal Health Record for Patients with Type 2 Diabetes Mellitus in Primary Care: A Research Protocol for a Backward and Forward Evaluation. in eTELEMED 2014, The Sixth International Conference on eHealth, Telemedicine, and Social Medicine. 2014.

2. Shaw, J.E., R.A. Sicree, and P.Z. Zimmet, Global estimates of the prevalence of diabetes for 2010 and 2030. Diabetes Research and Clinical Practice, 2010. 87(1): p. 4-14.

3. Klonoff, D.C., Using telemedicine to improve outcomes in diabetes-an emerging technology. Journal of diabetes science and technology (Online), 2009. 3(4): p. 624.

4. Bellazzi, R., Telemedicine and diabetes management: current challenges and future research directions. Journal of diabetes science and technology (Online), 2008. 2(1): p. 98.

5. Bodenheimer, T., et al., Patient self-management of chronic disease in primary care. JAMA, 2002. 288(19): p. 2469-2475.

6. Norris, S.L., et al., Recommendations for healthcare system and self-management education interventions to reduce morbidity and mortality from diabetes. American Journal of Preventive Medicine, 2002. 22(4 SUPPL. 1): p. 10-14.

7. Wagner, E.H., et al., Quality improvement in chronic illness care: a collaborative approach. The Joint Commission journal on quality improvement, 2001. 27(2): p. 63-80.

8. Verhoeven, F., et al., Asynchronous and Synchronous Teleconsultation for Diabetes Care: A Systematic Literature Review. Journal of Diabetes Science and Technology, 2010. 4(3): p. 666-684.

9. Goldberg, H.I., et al., Using an Internet comanagement module to improve the quality of chronic disease care. Joint Commission Journal on Quality and Patient Safety, 2003. 29(9): p. 443-451.

10. Tenforde, M., et al., The Association Between Personal Health Record Use and Diabetes Quality Measures. Journal of General Internal Medicine, 2012. 27(4): p. 420-424.

11. Osborn, C.Y., et al., Patient web portals to improve diabetes outcomes: A systematic review. Current Diabetes Reports, 2010. 10(6): p. 422-435.

12. Markle Connecting for Health. The Personal Health Working Group final report. July 1, 2003 Retrieved from: https://www.markle.org/publications/1429-personal-health-working-group-finalreport.

13. Tang, P.C., et al., Personal Health Records: Definitions, Benefits, and Strategies for Overcoming Barriers to Adoption. Journal of the American Medical Informatics Association, 2006. 13(2): p. 121126.

14. Pagliari, C., D. Detmer, and P. Singleton, Potential of electronic personal health records. BMJ, 2007. 335(7615): p. 330-333.

15. Tenforde, M., A. Jain, and J. Hickner, The value of personal health records for chronic disease management: what do we know? Family Medicine-Kansas City, 2011. 43(5): p. 351.

16. Black, A.D., et al., The Impact of eHealth on the Quality and Safety of Health Care: A Systematic Overview. PLoS Med, 2011. 8(1): p. e1000387.

17. Kaplan, B., Evaluating informatics applications-some alternative approaches: theory, social interactionism, and call for methodological pluralism. International journal of medical informatics, 2001. 64(1): p. 39-56.

18. Clamp, S. and J. Keen, Electronic health records: Is the evidence base any use? Informatics for Health and Social Care, 2007. 32(1): p. 5-10. 
19. Roelofsen, Y., et al., Design of the e-Vita diabetes mellitus study: Effects and use of an interactive online care platform in patients with type 2 diabetes (e-VitaDM-1/ZODIAC-40). Acta Veterinaria Scandinavica, 2014: p. 22.

20. Cain, M. and R. Mittman, Diffusion of innovation in health care. 2002: California Healthcare Foundation Oakland CA.

21. Van Gemert-Pijnen, J.E.W.C., et al., A holistic framework to improve the uptake and impact of eHealth technologies. Journal of medical Internet research, 2011. 13(4).

22. Corbin, J. and A. Strauss, Basics of qualitative research: Techniques and procedures for developing grounded theory. 2008: Sage.

23. Nazi, K.M., The personal health record paradox: Health care professionals' perspectives and the information ecology of personal health record systems in organizational and clinical settings. Journal of Medical Internet Research, 2013. 15(4).

24. Archer, N., et al., Personal health records: A scoping review. Journal of the American Medical Informatics Association, 2011. 18(4): p. 515-522.

25. Sieverink, F., et al., The Added Value of Log File Analyses of the Use of a Personal Health Record for Patients With Type 2 Diabetes Mellitus: Preliminary Results. Journal of Diabetes Science and Technology, 2014. 8(2): p. 247-255.

26. Van Gemert-Pijnen, J.E.W.C., O. Peters, and H.C. Ossebaard, Improving eHealth. 2013, The Hague, the Netherlands: Eleven International Publishing. 



\section{Chapter 6}

Evaluating the implementation of a Personal Health Record for chronic primary and secondary care

A mixed methods approach

F. Sieverink, S. Kelders, J. van Gemert-Pijnen (Submitted) 


\section{Abstract}

Background: eHealth, and more specifically Personal Health Records (PHRs), provide the opportunity for self-management support and maintaining and/or improving the quality of chronic disease management by engaging patients in their own healthcare. Despite the potential benefits of PHRs in chronic disease management, recent evidence regarding the effectiveness of PHRs for self-management remains sparse. Most of the evaluations focus on the effectiveness of PHRs as stand-alone, patient-centered technologies. The implementation of eHealth is a multi-level and complex process, and creating and implementing sustainable eHealth technologies thus requires a holistic development and evaluation approach that takes into account the triad of the technology, its users and the context of implementation.

Objectives: To gain a more complete understanding of what difference the PHR can make in the context of self-management support for the patients and the working routines of caregivers and why.

Methods: A convergent parallel mixed methods design was used, where qualitative and quantitative data were collected in parallel, analyzed separately, and finally merged. Log data was used to gain insight into how the PHRs were actually used on the long term. Focus group meetings were used to assess how caregivers believe that the use of the PHRs by patients adds value to their working routines. Interviews with caregivers and usability tests with potential end-users of the PHR were used to understand the differences and similarities between the intended and actual use of the PHR. All results were merged via an iterative process to find emerging themes, to consider these themes in the light of the research questions and to generate interpretive conclusions.

Results: The main problem that was identified was on the level of the context: A technology was offered to patients and their caregivers, without their involvement during earlier phases of development and without any guidance regarding the integration of the PHR into the daily working routines of caregivers. As a result, caregivers did not know what was expected from them with regards to using the services of PHR, and were ignorant on how to deploy these in daily care routines. The PHR was seen as a burden on top of the regular care and therefore, caregivers find it difficult to motivate their patients in using the PHR. During the focus groups, the coaching service was perceived as the core service of the PHR. However, the log data analyses showed that the actual use of the PHR by patients is lagging and did not match the intended use as indicated during the focus groups. It is likely that the usability of the PHR influenced the actual use as well.

Conclusion: The actual use of the PHRs by the patients was strongly influenced by the attitudes of caregivers. In turn, these attitudes are likely to be strongly influenced by the 
perceived support regarding the actual deployment the PHR in daily care routines. It is of utmost importance to involve all end-users already in early stages of eHealth development to increase the added value and to facilitate the implementation. Short and iterative development cycles are crucial for identifying problems already in early stages of development and to increase the chances for a successful implementation. When evaluating the implementation process, a mixed methods approach is of added value in providing explanations for the found effects that could not be revealed by solely focusing on the effectiveness of the technology in an experimental trial.

Keywords: Implementation, Personal Health Record, T2DM, CHF, COPD, mixed methods 


\section{Introduction}

In 2015, over 1.8 million people in the Netherlands were registered as having type 2 diabetes mellitus (T2DM), congestive heart failure (CHF) and/or COPD, and it is expected that these numbers will only grow in the upcoming years (approximately $11 \%$ of the Dutch population) [1-3].

With this growing prevalence, a number of issues arise. For example, due to a reimbursement shift from secondary to primary care, primary care providers (e.g., general practitioners and practice nurses) are responsible for a growing number of tasks concerning the treatment and counselling of chronically ill people. At the same time, the aging population causes a decrease in the number of care providers to deal with this shift. Furthermore, the relationship between patients and their care providers traditionally consists of a great dependence of the patient on the care provider, which can impose a costly burden [4]. Thereby, the healthcare system largely focuses on acute illnesses, while ageing with a chronic disease is becoming the norm [5]. This results in a mismatch with the needs of patients with chronic diseases for effective clinical management, psychological support, and information [6]. Sustainable solutions are needed to realize a transformation in health care delivery and to support the shifts from 1) institutionalized care to home care; 2) acute episodic care to a more continuous chronic care; and 3 ) the patient as a passive recipient of care to an active patient that is able to self-manage [7].

eHealth, and more specifically Personal Health Records (PHRs), provide the opportunity for self-management support and maintaining and/or improving the quality of chronic disease management by engaging patients in their own healthcare [8-10]. Originally, PHRs were defined as 'An electronic application through which individuals can access, manage, and share their health information and that of others for whom they are authorized, in a private, secure and confidential environment' [8]. To improve chronic health care and to support patients in developing self-management skills however, a PHR must be more than just a repository for managing and sharing health information [8]. Additional functions for supporting patients when working on health-related goals, learning more about (living with) chronic conditions and/or by supporting patient-provider communication can support patients in taking control over their own health [9].

Despite the potential benefits of PHRs in chronic disease management, recent evidence regarding the effectiveness of PHRs for self-management remains sparse [11, 12]. Services to support (a)synchronous communication between the diabetes patient and caregivers are associated with improved glycaemic control [13, 14], but the results regarding the effectiveness of other tools on improvements in clinical outcomes or the organization of care remain inconclusive $[13,15-18]$. This is often due to difficulties in the implementation [19]. 
Most of the evaluations focus on the effectiveness of PHRs as stand-alone, patient-centred technologies. However, according to the definition by Eysenbach [20], eHealth is much more than just a thing or a tool, but it is rather an infrastructure for knowledge dissemination, communication, and the organization of care. The implementation of eHealth is a multi-level and complex process $[19,21]$, and creating and implementing sustainable eHealth technologies thus requires a holistic development and evaluation approach that takes into account the triad of the technology, its users and the context of implementation. This hypothesis is supported by Nazi, who found that patient adherence to a PHR (using the PHR as intended) is likely to be directly influenced by the attitudes and actions of care providers [22]. The implementation of a PHR should therefore as well be seen in the greater context of the health care setting with a major role for the interplay between patients and caregivers. This implies that the deployment of a PHR with added value for both patients (e.g., selfmanagement support) and caregivers (e.g., disease management support) is a complex process that is influenced by several factors on multiple levels [19] that cannot be evaluated by focusing on only one level or outcome. However, up to now this holistic evaluation approach is often missing in current PHR research. In this study, we therefore focus on the interplay between the technology, its users and the context on the implementation of a PHR for patients with T2DM, CHF, or COPD in primary and secondary care. The research questions are as follows:

1. How did the attitudes and the actions of care providers regarding the PHR influence the actual use by patients?

2. To what extent does this actual use match with the use that adds value to the working routines in primary and secondary care, according to the caregivers (the intended use)?

3. How can any differences between the intended and actual use be understood?

To gain a more complete understanding of what difference the PHR can make in the context of self-management support for the patients and the working routines of caregivers and why, a mixed methods evaluation approach will be applied to combine quantitative and qualitative data on multiple levels [23].

\section{Methods}

\section{The e-Vita project}

Potentially significant savings in healthcare costs, together with a shortage of medical and healthcare professionals, are likely drivers for insurance companies and healthcare providers to invest in innovate approaches for chronic care management [24]. A partnership between Philips and Achmea, a Dutch health insurance company, made such an investment by developing the PHR e-Vita. The goal of this PHR was to improve the quality of life of 
chronically ill people and to improve the accessibility to care facilities [25]. Two PHRs were developed for T2DM and CHF. Based on these, a second version of the PHR was developed for patients with COPD.

The PHRs were implemented in primary care (T2DM and COPD) and secondary care (CHF) in the Netherlands for the duration of four trials to evaluate the effects of the PHR on the quality of life and health outcomes of patients with T2DM [26], CHF [27], and COPD [28]. The T2DM study included a randomized controlled trial to evaluate the effectiveness of a coaching service for self-management support [29]. A fifth research project was conducted to evaluate the impact of the PHR on health care utilization (cost-effectiveness).

The data described in this study are collected in the context of a sixth study, a process evaluation that incorporated all three PHRs. The goal of this study is to understand what differences PHRs can make in health care, why PHRs make these differences, and why PHRs may or may not have the expected impact. Therefore, not all the results from the process evaluation are used for this study; only the results that contributed to an answer to our research questions.

\section{System and content of the PHR}

The underlying system of the three PHRs was similar to a large extent, while the interfaces of the T2DM and CHF PHRs differed from the COPD PHR. Furthermore, the content differed depending on the condition. The PHR for CHF patients was linked to a system for telemonitoring. Patients were instructed to monitor their health values via that system on a daily basis, values were then transferred to the PHR. For the analyses in this study, we focus on the key features of the three PHRs as presented in Table 1. 
Table 1: An overview of the key-features of the PHRs

\begin{tabular}{|c|c|}
\hline Feature & Explanation \\
\hline $\begin{array}{l}\text { Insight in health- } \\
\text { related } \\
\text { measurements }\end{array}$ & $\begin{array}{l}\text { Users of the PHR for T2DM were able to see an overview (both } \\
\text { textually and graphically) of a selection of T2DM-related health } \\
\text { measurements from the past years, as performed at the annual } \\
\text { check-up. A short explanation of each item was provided to } \\
\text { support the participants with the interpretation of the values. }\end{array}$ \\
\hline Education & All three PHRs contained disease- and lifestyle-related education. \\
\hline Coaching & $\begin{array}{l}\text { The PHRs for T2DM and COPD offer the users the possibility to add } \\
\text { health-related goals, action plans and evaluations of these action } \\
\text { plans. The PHR for CHF did not contain this function. The coaching } \\
\text { function of the T2DM PHR was based on existing theories for } \\
\text { behavior change, literature research and previous experiences } \\
\text { and described elsewhere [29] }\end{array}$ \\
\hline Monitoring & $\begin{array}{l}\text { CHF patients received equipment to monitor their weight, blood } \\
\text { pressure, and heart rate on a daily basis (or individually adjusted } \\
\text { in concordance with the heart failure nurse). When the monitored } \\
\text { values were outside the pre-determined range or when no } \\
\text { measurements were recorded, HF nurses received an alert and } \\
\text { could subsequently contact the participant to possibly adjust the } \\
\text { (medical) treatment. } \\
\text { Patients with T2DM could track their health values using their own } \\
\text { equipment (e.g., weight, blood pressure, waist circumference). } \\
\text { Users with COPD could track their complaints and receive an } \\
\text { advice on whether or not to consult their care provider. } \\
\text { Furthermore, COPD users could regularly complete the Clinical } \\
\text { COPD Questionnaire (CCQ) [30] to gain insight into the } \\
\text { development of their clinical complaints. }\end{array}$ \\
\hline $\begin{array}{l}\text { Medication and } \\
\text { co-morbidities }\end{array}$ & $\begin{array}{l}\text { Users with CHF were asked to complete a list of medication and } \\
\text { co-morbidities and to keep this list up-to-date. For the HF nurses, } \\
\text { this overview could serve as an extra tool for the interpretation of } \\
\text { deviating measurements. }\end{array}$ \\
\hline Messaging & $\begin{array}{l}\text { The COPD PHR contained an overview of caregivers with a service } \\
\text { for sending and receiving messages to the caregivers. }\end{array}$ \\
\hline
\end{tabular}




\section{Training}

In all three trials, the caregivers (diabetes and COPD practice nurses in primary care and heart failure nurses in outpatient clinics) received the responsibility for introducing the PHR to their patients and to stimulate the subsequent use. For the T2DM PHR, nurses were trained to offer patients information and guidance with the use of the platform and the content [26]. For the CHF PHR, nurses received a $5 \mathrm{~h}$ training on the study procedures and adjustment of the range of the vital parameters [27]. For the COPD PHR, all caregivers received a refresher course based on an evidence based COPD disease management program and how to deploy the PHR in order to support the self-management skills of their patients [28].

\section{Previous evaluations of the PHRs}

Previous evaluations of e-Vita T2DM showed that of all registered users, 29.1\% logged in once, and 13.9\% logged in at least twice for at least 5 minutes per session [31]. Compared to participants who never logged in, these users had lower HbA1c levels and more often hypertension. Furthermore, these users reported a higher quality of life, better well-being, lower diabetes-related distress, and better medication adherence at baseline [31]. No effects could be shown of the T2DM PHR with self-management support. Of the 132 participants who agreed to participate in the study, only 5 participants used the coaching feature and 3 participants asked a coach for feedback on their set goals. No significant differences on any of the outcome measures were found after 6 months follow-up [32].

A log data analysis of the first use of the PHR showed that most users followed the structure of the technology when exploring the PHR. Users were likely to visit either 1 or 5 services during their first login, indicating the importance of the first impression. When users overcame this first impression, they made the effort to explore the rest of the PHR as well [33]. An interview study among the participating diabetes nurses in primary care indicated that a lack of integration of the PHR in daily care routines, the ability to try out the PHR with minimal investments and without commitment and the relative advantage in comparison to care routines without the deployment of a PHR were important factors for the diffusion of the PHR in primary care [34].

Evaluations of e-Vita CHF showed that of all patients who were eligible to participate in the study, the actual study participants were younger, more often male, healthier, and wealthier than patients who did not want to participate. The PHR improved self-care on the short term, but the effects diminished after 9 months follow-up [35]. However, an evaluation of the costeffectiveness showed that the PHR in combination with telemonitoring may provide health improvements at lowly costs [35].

Almost half of the participants (49.8\%) visited e-Vita for COPD once. Participants who received support in using the PHR (face-to-face or via telephone) logged in more frequently 
[36], but the COPD PHR had no impact on the health status of COPD patients, independently of the type of support [37].

To summarize, the evaluations of the PHR so far showed that the actual use of the PHR is lagging and only marginal health improvements were found. Reasons for the underperformance of the PHR remain unclear.

\section{Mixed methods study}

In the current study, a convergent parallel mixed methods design was used, where qualitative and quantitative data were collected in parallel, analysed separately, and finally merged to gain a more complete understanding of how the PHR was used in the context of selfmanagement support for the patients and the working routines of caregivers. Log data was used to gain insight into how the PHRs were actually used on the long term by patients with T2DM, CHF, or COPD. Results from focus group meetings were used to assess how caregivers believe that the use of the PHRs by patients adds value to their working routines (the intended use). Results from interviews with caregivers and usability tests with potential endusers of the PHR were used to understand the differences and similarities between the intended and actual use of the PHR. Ethical approval for all studies was obtained by the ethics committee of the University of Twente. All participants gave online or written consent to participate in this study.

\section{Log data}

To gain insight into the use of the PHRs for T2DM, CHF, and COPD throughout the study period, an analysis of log data (anonymous records containing information of every action by every user) was performed using a log data research protocol [38]. For every action, the following information was collected: 1) An anonymous identification of the user; 2) the time and date of the action; 3) an identification of the action, and 4) optional additional information (e.g., what information was viewed by the users or which goal was added). When logging in to the PHR, every user had to tick a box to accept the general conditions that contained a paragraph about tracking the usage for research purposes. When accepting these general conditions, users gave permission for collecting their usage data. The developers of the PHR facilitated the collection of log data. All data was stored and processed following the current privacy regulations. The log data of the T2DM PHR was collected from 23 July 2013 to 4 January 2016; the CHF PHR log data was collected from 9 October 2013 to 25 December 2015; and the COPD PHR log data was collected from 30 June 2014 to 29 May 2016.

For every user, sessions (the actions taken between logging in and logging out) were identified first. Due to technical reasons, logging out from the PHR could not be identified from the log data. Therefore, all actions that were performed within half an hour after the 
last action, were considered to be part of the same session. Next, only the actions that indicated visiting or using one of the PHR features (e.g., adding a health value, adding a goal or action plan, opening an education topic) were selected for the analysis. Per session, the frequencies of visiting and actually using the features were calculated.

\section{Focus groups}

To gain insight into to how the different services of the PHR can add value to the working routines of caregivers in order to be consistent with national standards for T2DM care, the data from two focus groups among 11 diabetes nurses and 2 dieticians were used. All participants were involved in the daily care for T2DM patients in primary care, but were not caregivers in the e-Vita T2DM trial.

During these focus groups, all participants were first asked to describe and concretize their current tasks and activities within the different phases of T2DM treatment, based on the national guidelines [39]. Second, all participants received a short explanation and examples of the possibilities of monitoring, coaching, education, and logistic support via a PHR, and were then asked to describe concretely how the use of these features could be supportive in a reduction of their workload and the stimulation of self-management skills among their patients.

Sound recordings were made of the focus groups and transcribed verbatim. The needs and wishes of the participants regarding the use of the potential key features of PHRs (monitoring, coaching, education, and logistic support) were then incorporated into a new care pathway for T2DM that includes the deployment of a PHR.

\section{Interviews and usability tests}

To gain insight into the implementation process of the PHR and to find explanations for any differences between the intended and actual use of the PHRs, the results from 28 semistructured, in-depth interviews among care providers who participated in the e-Vita trials were used.

Details regarding the procedure and results from the interviews among 11 T2DM caregivers are described elsewhere [34]. Furthermore, 9 caregivers in the CHF trial, and 8 caregivers in the COPD trial participated in the interview study. During the interviews, questions were asked regarding the reasons and incentives to use and implement a PHR in chronic care; experiences with the deployment of the PHR so far; encountered or expected bottlenecks and barriers for implementation; and the future of PHRs in chronic care. The duration of the interviews was 45-60 minutes.

To learn more about the experiences of patients regarding the use of the PHRs, 13 usability tests were conducted: 3 for the T2DM PHR and 10 for the COPD PHR. Since CHF patients had 
to perform their measurements using a system different from the PHR and the functionality of the PHR itself was limited, no usability tests for the CHF PHR were performed. Due to privacy issues it was not possible to invite the patient users of the PHRs. Therefore, usability tests among potential end-users were conducted. These participants were no real users of the PHR, but fitted within the profile of a real user with regard to age and/or chronic condition. The usability tests were conducted via a think-aloud protocol [40]. Examples of used scenarios are:

1. Imagine you want to lose $10 \mathrm{~kg}$ of weight. How would you add this goal to the PHR?

2. Today you measured your weight and you lost 2 kilograms. How would you add your new weight to the PHR?

3. When adding your weight, you made a typo error. How would you correct this in the PHR?

With the scenarios, all services of the PHRs as reported in Table 1 were tested. Where possible, the scenarios were kept the same across the different PHRs. Participants were stimulated to think aloud when conducting the tasks and were asked about their thoughts and feelings regarding the PHR afterwards. All interviews with the care providers and usability tests with potential end-users were transcribed verbatim. Themes and categories were coded via open coding, axial coding and selective coding.

\section{Synthesis of the results}

The results from the log data analyses, focus groups, interviews and usability tests were merged via an iterative process to find emerging themes, to consider these themes in the light of the research questions and to generate interpretive conclusions.

\section{Results}

\section{Log data analysis}

In the Figures 1-3, the visits and the actual use of the different services of the PHRs is shown for T2DM, CHF, and COPD, respectively. After their first login, 181 of the 301 T2DM users (60\%) returned for a second session and 50/301 users (17\%) returned for six or more sessions. Half of all COPD users returned for six or more sessions. Since CHF patients were asked to perform their measurements on a daily basis, the results of the analyses show that the CHF PHR was used on a longer term. Therefore, the first 200 sessions are shown in Figure 2. Ninety-one of $147 \mathrm{CHF}$ users (62\%) returned for a second session, 16/147 users (11\%) returned for 101 or more sessions. Across the three PHRs, all services are viewed by the users, but the actual usage is lagging. 
The education services were used the most. For the T2DM PHR, educational topics were on average opened by $20 \%$ of the users across the first six sessions, for the COPD PHR this percentage was 3\%. On average, almost half of the CHF users (48\%) actually opened the educational topics across the first 200 sessions. CHF patients used this education service together with the overviews of monitored values and target values, while COPD patients used the overview of healthcare providers as well. The coaching service was used the least by T2DM and COPD patients, CHF patients used the service for adding medication the least.

For the T2DM PHR, the use of the education service increased after the fourth session, while the use of the monitoring service is slightly decreasing. In $\mathrm{CHF}$, the percentage of individuals that use the monitoring overviews is slightly increasing, indicating more targeted use when individuals return on the long term. The actual use of the education services is the least in sessions 41 to 50, but increases again after 50 sessions.

The use of the different services by the COPD patients is the highest in the first session, which was a training session. After the first session, the actual use of the different services decreases, but stays more or less constant over time. In comparison with the other PHRs, the service for education was relatively less used by the COPD users. 


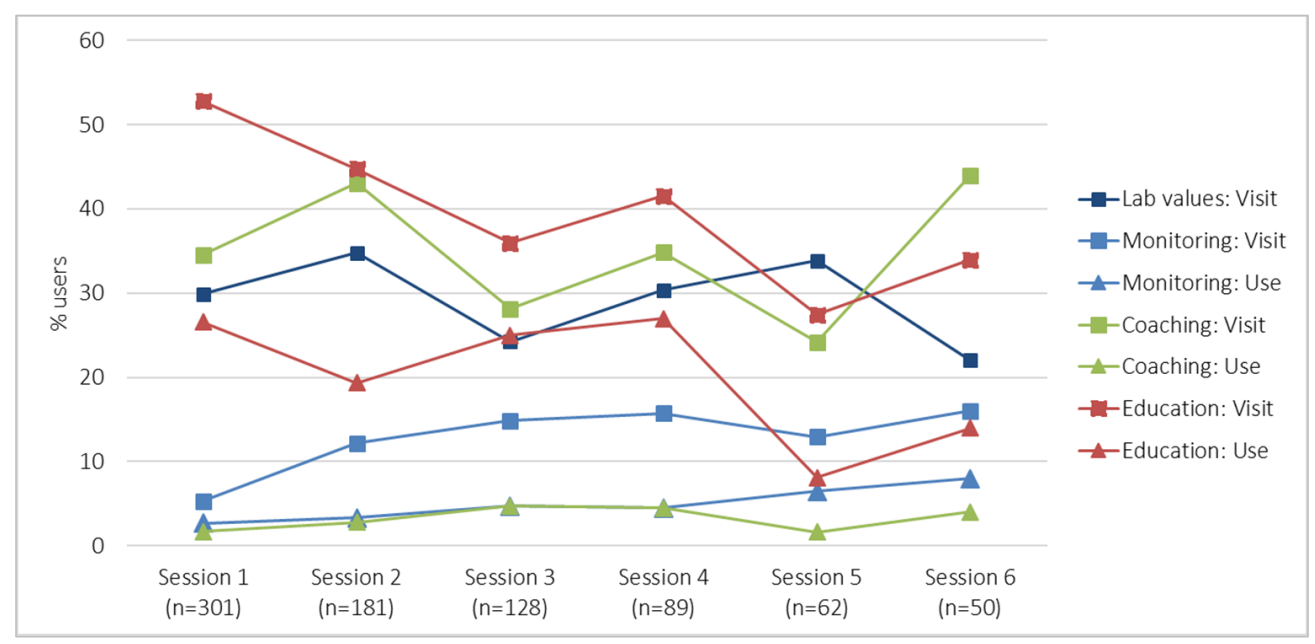

Monitoring action - Adding a new measurement, opening the overview of target values and monitoring history. Coaching action: adding a wish, goal, action or evaluation. Education action: opening education topic. No specific actions could be specified for lab values.

$\%$ users $=$ (number of different users visiting or using the feature in that session / total number of users in that session) * 100

Figure 1. Usage per session and feature (T2DM)

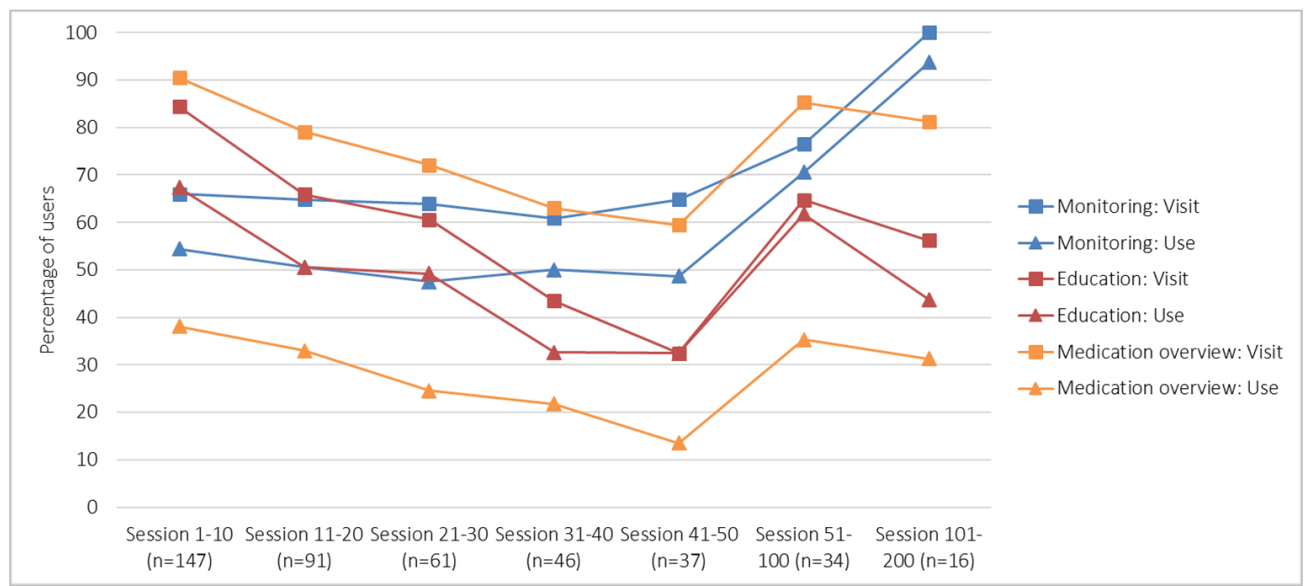

Monitoring action - Adding a new measurement, opening the overview of target values and monitoring history. Coaching action: adding a wish, goal, action or evaluation. Education action: opening education topic. No specific actions could be specified for lab values.

$\%$ users $=$ (number of different users visiting or using the feature in that session / total number of users in that session) * 100

Figure 2. Usage per session and feature (CHF) 


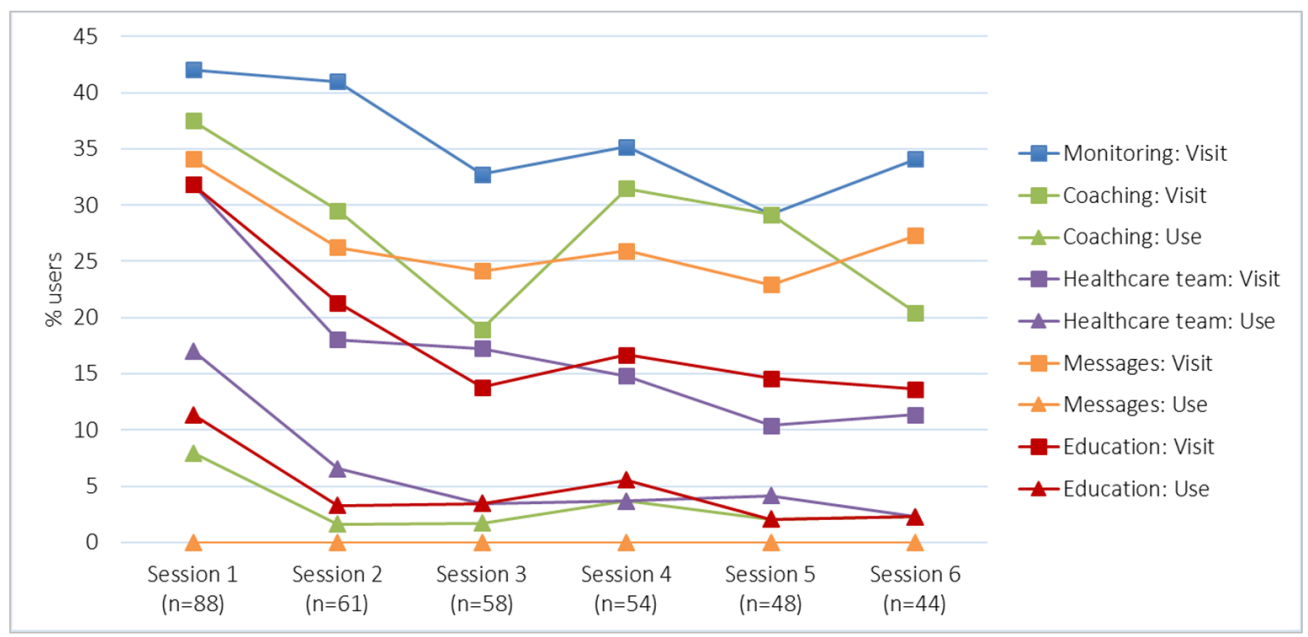

Monitoring action - Adding a new measurement, opening the overview of target values and monitoring history. Coaching action: adding a wish, goal, action or evaluation. Education action: opening education topic. No specific actions could be specified for lab values.

$\%$ users $=$ (number of different users visiting or using the feature in that session / total number of users in that session) * 100

Figure 3. Usage per session and feature (COPD)

\section{Focus groups}

Based on the results of the focus groups, an example of a care pathway with the integration of a PHR was created (Figure 4). The participants of the focus groups indicated that they mainly see added value in the services for monitoring health values such as blood pressure, weight, and blood glucose levels during the day. When the caregiver has access to the data, there is no need to repeat the measurements during the consultation, which can be timesaving. The selection and the frequency of measurements depends, among others, on the treatment goal and the personal goals of the patient and should therefore be determined in consultation with the patient. The selection and frequency of measurements in Figure 4 is therefore indicative. According to the caregivers, performing the measurements at home using valid and reliable tools is an important prerequisite.

Education is seen as an important service of the PHR and is strongly related with the monitoring plan and the personal health-related goals of the patient. In the current situation (without the use of a PHR), caregivers spend much time educating their patients. However, when a caregiver knows the individual goals and the motivation of the patient and what the patient does (not) know, patients can be directed towards the educational topics on the PHR that are of added value at that moment. Thereby, the education service can be time-saving for caregivers. Coaching is strongly associated with monitoring health values (to gain insight 
into the progression towards the health-related goal) and education (what the patient needs to know to be able to reach a certain goal).

Caregivers indicate that is important for patients to contemplate about their health-related goals before a consultation and to add them to a PHR, since this will help to organize their thoughts regarding their goals, which can in turn support self-management. During a consultation, the caregiver can support the patient in formulating achievable goals. According to the caregivers, the personal goals should be leading during the consultations to keep the patient motivated. They also find it important to make arrangements with the patients about if and when feedback is given on evaluations within consultations, to avoid disappointments and demotivation.

As an additional function, caregivers indicated that they see added value in offering preconsultations via the PHR. They experience that their patients sometimes find it hard to talk about certain topics, or forget to mention important information during consultations. According to the caregivers, pre-consultations should include the following topics: complaints since the last consultation, medication use, a questionnaire to address depressive complaints, medical (family) history, and co-morbidities. 


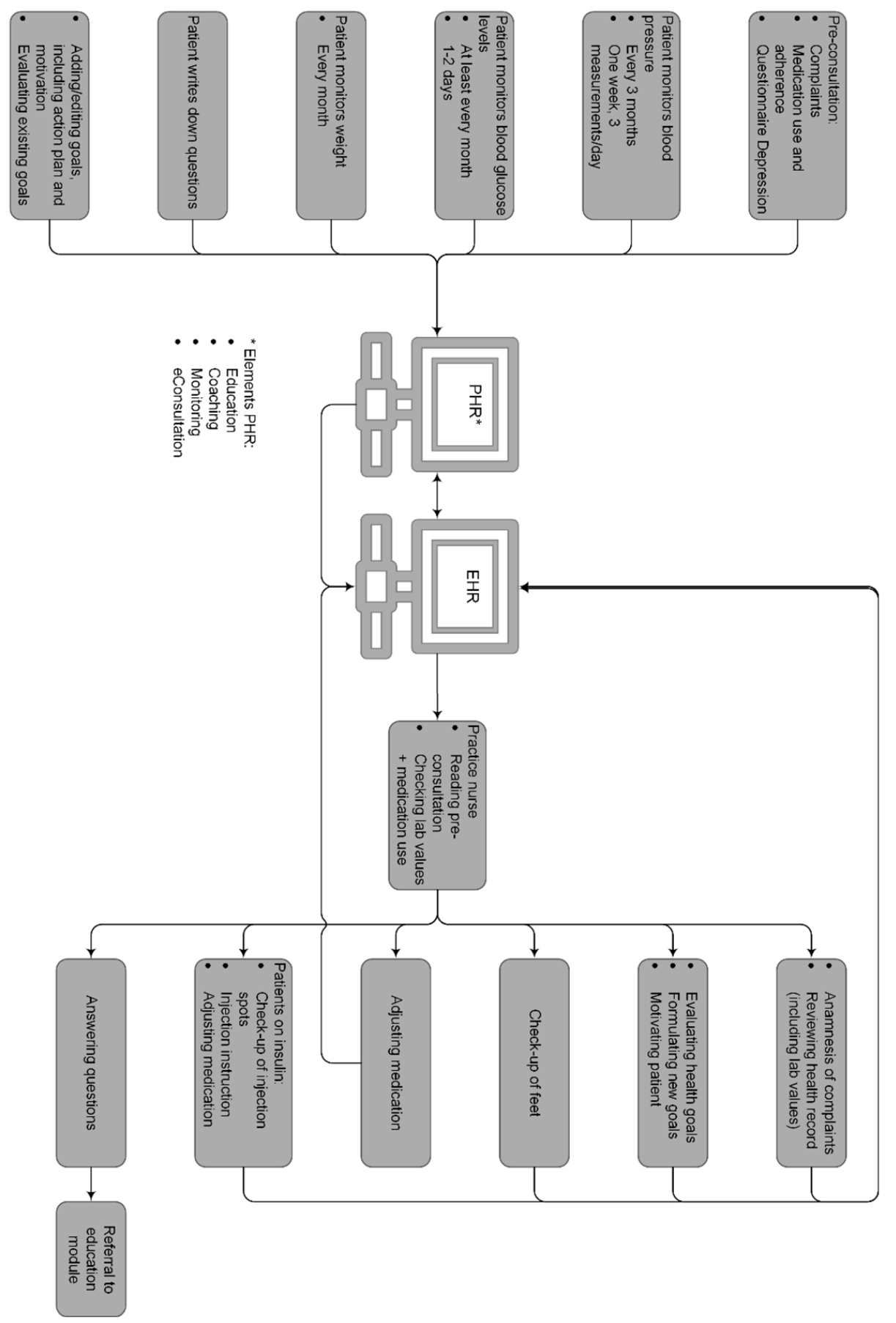

Figure 4. An example of a care pathway with the integration of a PHR for T2DM for the follow-up consultations in primary care. 


\section{Interview study}

Although all participating caregivers in the interview study were mainly enthusiastic regarding the use of a PHR in chronic care in order to support the self-management skills of their patients, there were still some issues that hindered a successful implementation of the PHR in daily care routines (Table 3 ).

\section{Training and guidance}

All participating nurses (in all three groups) were trained once or twice in the services the PHRs offer and the protocols for the evaluation studies. After these training sessions, the nurses mostly felt that the functions of the PHR were easy to use, but they did not feel they had a comprehensive grasp on how to integrate the PHR into their daily care routines.

\section{Compatibility with other systems}

An important and frequently raised issue among all participating caregivers, was the incompatibility of the PHR with the other systems the caregivers had to work with. All caregivers are using at least an EHR and an email client in their daily working routines as well, between which they often had to switch. This was perceived as impractical and labourintensive. The caregivers in the CHF project for example, were asked to check the monitored health values of their patients on a daily basis in the telemonitoring system. When values of a certain patient were deviating, the HF nurses first needed to access the PHR to gain insight into changes in medication and/or co-morbidities. Subsequently, the patient had to be contacted and every follow-up action needs to be registered in the EHR as well.

\section{Integration in daily routines}

A third barrier in the deployment of the PHRs was the lack of integration in daily care routines. Among the care providers, this could either result in forgetting to use the PHR, or in seeing the PHR as an time- and effort consuming technology instead of a technology to support daily tasks. As a result, the services of the PHRs were barely used during consultations with the patients. Also, HF nurses indicated that they often cooperate with other healthcare facilities (such as GPs or nursing homes) to provide the required care for $\mathrm{CHF}$ patients. The PHR should as well be embedded in these structures in order to provide the required care for the patients.

However, care providers do expect that, when the PHR is better integrated, their workload will reduce; patients will be stimulated to take their own responsibilities; and selfmanagement will be stimulated.

\section{Motivating patients}

Some caregivers indicated that only a small fraction of their patient population is using the $\mathrm{PHR}$, and only a very small proportion of this group is an active user. Caregivers indicated 
that they therefore found it hard to establish new working routines, and using the PHR was therefore perceived as time-consuming. Furthermore, the caregivers often did not recognize the added value of the PHR for their patients. Due to technical problems and the unavailability of test accounts, caregivers did not know for a long time what the PHR looked like for their patients. As a result, caregivers found it hard to motivate their patients in using the PHR. Another example comes from the T2DM project, where the majority of caregivers was not aware of the fact that their patients only gained insight into their most recent lab values as soon as the caregiver uploaded these results into a regional benchmarking system. Therefore, these results were not always up-to-date for the patients. Furthermore, as previously described, the medication overviews that CHF users are supposed to keep up-todate via the PHR could serve as an additional information source for caregivers when deviating telemonitoring values are being signaled. However, the caregivers experienced that these overviews are often not accurate, not kept up-to-date, or not filled in at all. Therefore, HF nurses indicated that it is more efficient to directly call the patient when values are deviating, instead of checking the overviews first.

\section{Usability}

The usability of the PHRs and the visualization of data for caregivers are seen as important barriers for the implementation as well. The PHR is perceived as slow and unclear, and instructions on the COPD PHR were sometimes perceived as patronizing. Furthermore, patients and caregivers often did not receive their login credentials or logging in was not possible due to technical issues.

\section{Communication via the PHR}

Caregivers in the three research groups indicated that they would value the opportunity for short interactions with their patients via the PHRs, for example via a chat or messaging function. Furthermore, the caregivers see possibilities for improving the interaction between the users and the PHRs. They noted that patients are sometimes left with unanswered questions, for example when they see deviating health values. Users should then be able to find an answer via the PHR to the first questions they may have (e.g., are values indeed deviating, and why could that be? What actions can be taken now?). Furthermore, HF nurses indicate that they often ask the same questions to their patients in case telemonitoring values are deviating. A short questionnaire comprising health-related questions for patients to complete after every measurement would increase the efficiency of the telemonitoring system and help the HF nurses to increase the accuracy of the decisions that are made based on the telemonitoring values. 
Table 3. Topics that influenced the implementation of the PHR, according to care providers

\begin{tabular}{|c|c|}
\hline Topic & Example \\
\hline \multirow[t]{4}{*}{$\begin{array}{l}\text { Training and } \\
\text { guidance }\end{array}$} & $\begin{array}{l}\text { 'Yes, that was not really difficult. The PHR, seeing health values, it is not } \\
\text { very difficult.' }\end{array}$ \\
\hline & $\begin{array}{l}\text { '...No idea how I had to work so we sat with the manual next to the patient, } \\
\text { we went through it step by step. But then the patient also had enough of } \\
\text { it, no matter how positively you started it, at some point you notice your } \\
\text { own frustration.' }\end{array}$ \\
\hline & 'It was still vague what exactly we had to do and what was expected of us.' \\
\hline & $\begin{array}{l}\text { 'Well, after training you still need to try it yourself and look at the platform } \\
\text { and the PowerPoint again. You need to show initiative by yourself, } \\
\text { otherwise it is difficult.' }\end{array}$ \\
\hline \multirow[t]{3}{*}{$\begin{array}{l}\text { Compatibility } \\
\text { with other } \\
\text { systems }\end{array}$} & $\begin{array}{l}\text { 'At this time, we work with three different systems: the PHR, our own EHR, } \\
\text { and the telemonitoring system. We constantly need to switch between } \\
\text { these three systems, that is just difficult. It is just not workable that way.' }\end{array}$ \\
\hline & $\begin{array}{l}\text { 'It should be user-friendly. You should not have four systems in which you } \\
\text { have to fill the same information.' }\end{array}$ \\
\hline & $\begin{array}{l}\text { 'We must keep track of two systems, which is time consuming, it is not } \\
\text { possible to have a link between the PHR and the EHR at the same time' }\end{array}$ \\
\hline \multirow{2}{*}{$\begin{array}{l}\text { Integration in } \\
\text { daily care } \\
\text { routines }\end{array}$} & $\begin{array}{l}\text { 'I must honestly say that we forget to look at the PHR. That is also because } \\
\text { it is not in your daily routine.' }\end{array}$ \\
\hline & $\begin{array}{l}\text { 'I don't feel like going looking there each day, but maybe once every } \\
\text { month to take a look at what I can improve and how. }\end{array}$ \\
\hline \multirow[t]{2}{*}{ Use by patients } & 'They are often also excited but in practice they still do not use it.' \\
\hline & $\begin{array}{l}\text { 'Because he did not fill in the medication and co-morbidities, while we } \\
\text { clearly stated during inclusion, that they had to fill in so we could take it } \\
\text { into account when health values deviate. The first person did not do it, the } \\
\text { second neither, then you fall back on your own system.' }\end{array}$ \\
\hline \multirow[t]{2}{*}{ Usability } & $\begin{array}{l}\text { 'There were a lot of things in this platform, that did not work. In addition, } \\
\text { it was not really an enchanting site and especially in this hospital very slow. } \\
\text { We did not really work with the PHR, I must say.' }\end{array}$ \\
\hline & $\begin{array}{l}\text { 'You can see recent values, and the last two weeks. That is also a } \\
\text { limitation, because sometimes you want to see for a longer term.' }\end{array}$ \\
\hline \multirow[t]{2}{*}{$\begin{array}{l}\text { Communication } \\
\text { via PHR }\end{array}$} & $\begin{array}{l}\text { 'Implement a certain learning element. For example, that the system } \\
\text { indicates: "You have now gained two pounds, do you think that the care } \\
\text { giver should take action or are you capable to do something on your } \\
\text { own?"' }\end{array}$ \\
\hline & $\begin{array}{l}\text { 'For example, a digital contact with the patient. Especially with younger } \\
\text { patients, or people who are used to e-mail. Contact them via this way.' }\end{array}$ \\
\hline
\end{tabular}




\section{Perceived usability among potential end-users}

The usability tests among three potential T2DM end-users showed that they perceive the PHR as appealing and inviting at first sight. However, they did expect a welcome message and a short explanation regarding the goal and the features of the PHR. As soon as the potential end-users were invited to execute the scenarios, it became clear that the structure of the system did not match their expectations. For example, they expected a function for monitoring blood glucose levels, and within the coaching feature, the concepts of 'wishes', 'goals' and 'actions' remained unclear. Furthermore, they found it confusing that some elements (e.g., videos and some information topics) were opened in a new window.

The 10 usability tests among potential end-users of the COPD PHR revealed that participants experienced difficulties with finding the right information. Because there are many buttons in one screen, the lay-out was perceived as unclear. Some elements at the home-page look like buttons but are not, which causes confusion. Furthermore, the coaching function is perceived as unclear. For the participants, the distinction between different concepts (e.g., wish, challenge) is unclear, as well as how to add wishes, action plans, and evaluations to the PHR. Participants value the overview of care providers. However, when participants select a caregiver to send a message, the message screen opens out of sight of the participants.

The participants in both the T2DM and COPD groups indicate that they would like to use the PHR in the future, providing that the usability will be improved and that the caregivers will use it in daily practice as well. In that case, participants would value a replacement of regular consultations as well. Especially the possibility to monitor health values is appreciated. Participants prefer to look up additional information regarding their condition via search engines such as Google.

\section{Synthesis}

From the interviews with the caregivers, some important factors influencing the actual use could be identified. Prior to the start of the project, no intended use was formulated for the T2DM and COPD PHRs for both the patients and the caregivers and the training mainly focused on the infrastructure of the research projects or the technical functionalities of the different services. As a result, caregivers perceived the support in the actual integration of the PHR into their working routines as insufficient. Caregivers often stressed that they are ignorant on how to deploy the different services of the PHR in such a way that they are of added value in daily care routines. Furthermore, it remained unclear what was expected from them throughout the research project, which influenced their motivation to participate. Therefore, they perceive the PHR as an additional task on top of their current routines. These results can serve as an explanation for most patients using the PHRs for only one session: because it was not integrated in the working routines of caregivers, patients seemed not to see the added value as well. 
Only a small number of all T2DM and COPD users have actually added health-related goals and measurements to the PHRs. This could be due to the possibility that they do not see added value in these services because they are not motivated by their caregivers and the shared information is not used in their treatment. It could also be that they perceive the terminology on the PHR as unclear, as identified by the usability tests among end-users.

The actual use of the PHRs does not match the opinions of caregivers about how to integrate the different services of a PHR within their working routines. They indicated that within the treatment of chronically ill, the health-related goals of the patients should be leading and that a monitoring and education plan should be formulated in concordance with the patients, but this was not seen in the usage data. Furthermore, an important result of the focus group study is that caregivers see added value in using health measurements and other information provided by the patients when creating treatment plans. However, only a very small percentage of T2DM users added their health-related measurements to the PHR and the medication overview was used the least by the CHF users. Moreover, none of the caregivers that participated in the PHR trials indicated that they actually stimulate their patients in using the PHR that way.

\section{Discussion}

The aim of this study was to gain insight into the interplay between the technology, its users and the context of implementation of a PHR for patients with T2DM, CHF, or COPD in primary and secondary care. To get a more complete understanding of how the PHR was used in the context of self-management support for the patients and the working routines of caregivers, a convergent parallel mixed methods evaluation was used to merge quantitative and qualitative data via an iterative process on multiple levels. By this, we gained a more complete understanding of how the attitudes and actions of care providers influenced the actual use of the PHRs by patients; to what extent the actual use matched the use that adds value to the working routines in primary and secondary care, according to caregivers (the intended use), and how any differences between the actual and intended use could be explained.

\section{Principal Results}

The results showed that with the development and implementation of the PHR, insufficient attention has been paid to the triad of the user, the technology, and the context. The main problem that was identified was on the level of the context: A technology was offered to patients and their caregivers, without their involvement during earlier phases of development and without any guidance regarding the integration of the PHR into the daily working routines of caregivers. The PHR was mainly introduced in the light of the evaluation study and the training of the caregivers predominantly focused on how to collect the data 
for this study. As a result, caregivers did not know what was expected from them with regards to using the services of PHR, and were ignorant on how to deploy these in daily care routines. Because the workload is perceived as high, caregivers experienced that they had insufficient time to explore what the PHR could potentially mean for their working routines and as a result, the PHR was seen as a burden on top of the regular care. Therefore, caregivers find it difficult to motivate their patients in using the PHR, and the log data analyses showed that the actual use of the PHR by patients is lagging as well. This limited use is common in PHR evaluations [41] and several recent systematic reviews focusing on the implementation of complex (eHealth) interventions and PHRs stress that the (perceived) fit of eHealth technologies with the current working routines and the interoperability with other systems are key factors for a successful implementation [19, 41, 42]. In turn, an engaged caregiver might be the key to patient engagement [43-45].

Moreover, it is likely that the usability of the PHR influenced its actual use as well. Many T2DM caregivers indicated prolonged troubles with logging in and participants of the usability tests perceived the designation of the concepts within the coaching service (e.g., Wish, Goal, and Action) as unclear and uninviting to work with. This finding might explain why the coaching service (which was perceived as the 'core' service of the PHR during the focus group meetings) was used by so few users.

\section{Implications and recommendations}

The results of this study show that a holistic approach of eHealth development, implementation, and evaluation is crucial in the success of the project. eHealth is not just a tool which will be implemented automatically once it is offered to the end-users without any considerations. Rather, the whole of the interactions between the system, the user, and the context in which the eHealth technology is deployed is of utmost importance. These results are consistent with previous systematic reviews focusing on eHealth implementation, stressing that the (perceived) fit of eHealth technologies with the current working routines and the interoperability with other systems were key factors for a successful implementation $[19,42]$. Furthermore, the findings of the current studies are supported by Nazi, who emphasizes the role of the caregivers in the adoption of PHRs by the patients [22]. Therefore, decent training and support of all end-users in deploying the system is necessary, but when the first impression is modest and the technology does not fit the wishes, needs, and the context of end-users (and the added value is thus lacking), it will hardly or not be used.

Therefore, it is important to involve all stakeholders, including the end-users, already in early stages of development to gain insight into their needs and wishes. However, the inquiry of the context is just as important $[21,46]$, for example by exploring national guidelines or by observing the daily (working) routines of caregivers and patients. Short and iterative development cycles addressing the technology and its interaction with the users and the 
context are crucial [42], because it allows for problems to be identified already early in the development, increasing the chances for a successful implementation $[21,46]$.

To be able to understand what differences a technology can make and why some technologies are effective whilst others are not, data from differences sources in a mixed methods approach has proven to be of added value [23]. In this study, focusing on (the relations between) multiple levels provided explanations for the disappointing results of eVita so far, that could not be revealed by solely focusing on the effectiveness of the technology in an experimental trial. In this research, the log data had the potential to provided objective insights into the actual usage of the different services of e-Vita, but could only be put into perspective by using the results from the focus group study, interview studies and usability tests [38]. At the same time, only focusing on the experiences of caregivers would not have given insight into the influence on the actual usage of the PHR. Thus, understanding the interplay between the technology, its users and the context of implementation are of utmost importance when understanding the effectiveness.

\section{Limitations}

An important limitation of this research was that during the focus groups, only insights from T2DM caregivers could be obtained. This might have decreased the generalizability of the results with respect to the other disease groups. However, because we focused on the aspects that are similar between disease groups and used a mixed methods approach, we could extrapolate these finding to $\mathrm{CHF}$ and COPD.

Furthermore, because of the large number of participants across the three trials, we were able to interview only a small proportion of a diverse population. However, despite this variation, no new insights were obtained from the last conducted interviews and we therefore feel that saturation has been reached. Furthermore, by combining the interview data with objective log data from all users in the mixed methods approach, we were able to cope with this potential limitation.

\section{Conclusions}

In this study, the actual use of the PHRs by the patients is strongly influenced by the attitudes of caregivers. In turn, these attitudes are likely to be strongly influenced by the perceived support regarding the actual deployment the PHR in daily care routines. Moreover, the actual use did not match the intended use according to the caregivers. It could thus be concluded from the results of this study that it is of utmost importance to involve all end-users already in early stages of eHealth development to increase the added value and to facilitate the implementation. Short and iterative development cycles are crucial for identifying problems already in early stages of development and to increase the chances for a successful implementation. When evaluating the implementation process, a mixed methods approach 
is of added value in providing explanations for the found effects that could not be revealed by solely focusing on the effectiveness of the technology in an experimental trial.

\section{Acknowledgements}

The authors would like to thank Pieter Jeekel, Vital Health Software and Coert Blom for their support in collecting the log data; prof. Henk Bilo, Yvonne Roelofsen, Steven Hendriks, Kim Wagenaar, prof. Arno Hoes, Esther Talboom, Anneke Vass, and prof. Niels Chavannes for facilitating the interview studies; and Ingeborg Weuring and Mirande Groener for facilitating the focus group study. Furthermore, the authors would like to thank Stefan Olde Olthof and Anita Borgerink for their contribution in the data collection and analyses of the interview studies. 


\section{References}

1. Baan, C.A. and M.J.J.C. Poos. Diabetes mellitus - Cijfers en Context - Huidige Situatie. Available from: https://www.volksgezondheidenzorg.info/onderwerp/diabetes-mellitus/cijfers-context/huidigesituatie.

2. Engelfriet, P.M., M.J.J.C. Poos, and F.H. Rutten. Hartfalen - Cijfers en Context - Huidige Situatie. Available from: https://www.volksgezondheidenzorg.info/onderwerp/hartfalen/cijferscontext/huidige-situatie.

3. Poos, M.J.J.C. and M. Nielen. COPD - Cijfers en Context - Huidige Situatie. Available from: https://www.volksgezondheidenzorg.info/onderwerp/copd/cijfers-context/huidige-situatie.

4. Roehrs, A., et al., Personal Health Records: A Systematic Literature Review. J Med Internet Res, 2017. 19(1): p. e13.

5. Huber, M., et al., How should we define health? BMJ, 2011. 343.

6. Wagner, E.H., et al., Improving Chronic Illness Care: Translating Evidence Into Action. Health Affairs, 2001. 20(6): p. 64-78.

7. Organization, W.H., Innovative care for chronic conditions: building blocks for actions: global report. 2002.

8. Tang, P.C., et al., Personal Health Records: Definitions, Benefits, and Strategies for Overcoming Barriers to Adoption. Journal of the American Medical Informatics Association, 2006. 13(2): p. 121126.

9. Pagliari, C., D. Detmer, and P. Singleton, Potential of electronic personal health records. BMJ, 2007. 335(7615): p. 330-333.

10. Gee, P.M., et al., The eHealth Enhanced Chronic Care Model: A Theory Derivation Approach. J Med Internet Res, 2015. 17(4): p. e86.

11. Johansen, M.A. and E. Henriksen, The Evolution of Personal Health Records and their Role for SelfManagement: A Literature Review, in Studies in Health Technology and Informatics. 2014. p. 458462.

12. Goldzweig, C.J., et al., Electronic Patient Portals: Evidence on Health Outcomes, Satisfaction, Efficiency, and Attitudes - A Systematic Review. Annals of Internal Medicine, 2013. 159(10): p. 677687.

13. Coughlin, S.S., L.B. Williams, and C. Hatzigeorgiou, A systematic review of studies of web portals for patients with diabetes mellitus. mHealth, 2017. 3(6).

14. Goldzweig, C.L., et al., Secure Messaging between Providers and Patients, and Patients' Access to Their Own Medical Record. 2012.

15. Osborn, C.Y., et al., Patient web portals to improve diabetes outcomes: A systematic review. Current Diabetes Reports, 2010. 10(6): p. 422-435.

16. Kooij, L., W.G. Groen, and W.H. Van Harten, The effectiveness of information technology-supported shared care for patients with chronic disease: A systematic review. Journal of Medical Internet Research, 2017. 19(6).

17. Giardina, T.D., et al., Patient access to medical records and healthcare outcomes: A systematic review. Journal of the American Medical Informatics Association, 2014. 21(4): p. 737-741.

18. Tenforde, M., A. Jain, and J. Hickner, The value of personal health records for chronic disease management: what do we know? Family Medicine-Kansas City, 2011. 43(5): p. 351. 
19. Ross, J., et al., Factors that influence the implementation of e-health: a systematic review of systematic reviews (an update). Implementation Science, 2016. 11(1): p. 146.

20. Eysenbach, G., CONSORT-EHEALTH: Improving and Standardizing Evaluation Reports of Web-based and Mobile Health Interventions. J Med Internet Res, 2011. 13(4): p. e126.

21. Glasgow, R.E., S.M. Phillips, and M.A. Sanchez, Implementation science approaches for integrating eHealth research into practice and policy. International Journal of Medical Informatics, 2014. 83(7): p. e1-e11.

22. Nazi, K.M., The Personal Health Record Paradox: Health Care Professionals' Perspectives and the Information Ecology of Personal Health Record Systems in Organizational and Clinical Settings. J Med Internet Res, 2013. 15(4): p. e70.

23. Creswell, J.W., Research design: Qualitative, quantitative, and mixed methods approaches. 2013: Sage publications.

24. van Gemert-Pijnen, J., O. Peters, and H.C. Ossebaard, Improving eHealth. 2013: Eleven international publishing Den Haag, The Netherlands.

25. Stichting Zorg Binnen Bereik, available from: http://www.zorgbinnenbereik.nl/over-ons/missie-envisie.html.

26. Roelofsen, Y., et al., Design of the e-Vita diabetes mellitus study: effects and use of an interactive online care platform in patients with type 2 diabetes (e-VitaDM-1/ZODIAC-40). BMC Endocrine Disorders, 2014. 14(1): p. 22.

27. Wagenaar, K.P., et al., Effectiveness of an interactive platform, and the ESC/HFA heartfailurematters.org website in patients with heart failure: design of the multicentre randomized e-Vita heart failure trial. European Journal of Heart Failure, 2015. 17(12): p. 1310-1316.

28. Talboom-Kamp, E.P.W.A., et al., e-Vita: design of an innovative approach to COPD disease management in primary care through eHealth application. BMC Pulmonary Medicine, 2016. 16(1): p. 121.

29. Van Vugt, M., et al., Web-based self-management with and without coaching for type 2 diabetes patients in primary care: design of a randomized controlled trial. BMC Endocrine Disorders, 2013. 13(1): p. 53.

30. Van der Molen, T., et al., Development, validity and responsiveness of the Clinical COPD Questionnaire. Health and Quality of Life Outcomes, 2003. 1(1): p. 13.

31. Roelofsen, Y., et al., Demographical, Clinical, and Psychological Characteristics of Users and Nonusers of an Online Platform for T2DM Patients (e-VitaDM-3/ZODIAC-44). Journal of Diabetes Research, 2016. 2016: p. 16.

32. Van Vugt, M., et al., Uptake and Effects of the e-Vita Personal Health Record with Self-Management Support and Coaching, for Type 2 Diabetes Patients Treated in Primary Care. Journal of Diabetes Research, 2016. 2016: p. 9.

33. Sieverink, F., et al., The Added Value of Log File Analyses of the Use of a Personal Health Record for Patients With Type 2 Diabetes Mellitus. Journal of Diabetes Science and Technology, 2014. 8(2): p. 247-255.

34. Sieverink, F., et al., The Diffusion of a Personal Health Record for Patients with Type 2 Diabetes Mellitus in Primary Care. International Journal on Advances in Life Sciences, 2014. 6(3\&4): p. 177 183.

35. Wagenaar, K., E-health in heart failure. 2017, University Utrecht. 
36. Talboom-Kamp, E.P., et al., High Level of Integration in Integrated Disease Management Leads to Higher Usage in the e-Vita Study: Self-Management of Chronic Obstructive Pulmonary Disease With Web-Based Platforms in a Parallel Cohort Design. J Med Internet Res, 2017. 19(5): p. e185.

37. Talboom-Kamp, E.P., et al., The Effect of Integration of Self-Management Web Platforms on Health Status in Chronic Obstructive Pulmonary Disease Management in Primary Care (e-Vita Study): Interrupted Time Series Design. J Med Internet Res, 2017. 19(8): p. e291.

38. Sieverink, F., et al., Opening the black box of eHealth: Collecting, analyzing, and interpreting log data. JMIR Research Protocols, 2017.

39. Rutten, G., et al., NHG-Standaard Diabetes mellitus type 2 (derde herziening). Huisarts Wet, 2013. 56(10): p. 512-525.

40. Jaspers, M.W.M., A comparison of usability methods for testing interactive health technologies: Methodological aspects and empirical evidence. International Journal of Medical Informatics, 2009. 78(5): p. 340-353.

41. Otte-Trojel, T., et al., What do we know about developing patient portals? a systematic literature review. Journal of the American Medical Informatics Association, 2016. 23(e1): p. e162-e168.

42. Lau, R., et al., Achieving change in primary care-causes of the evidence to practice gap: systematic reviews of reviews. Implementation Science, 2016. 11(1): p. 40.

43. Krist, A.H., et al., Engaging Primary Care Patients to Use a Patient-Centered Personal Health Record. The Annals of Family Medicine, 2014. 12(5): p. 418-426.

44. Amante, D.J., et al., A Systematic Review of Electronic Portal Usage Among Patients with Diabetes. Diabetes Technology \& Therapeutics, 2014. 16(11): p. 784-793.

45. Agarwal, R., et al., If We Offer it, Will They Accept? Factors Affecting Patient Use Intentions of Personal Health Records and Secure Messaging. J Med Internet Res, 2013. 15(2): p. e43.

46. Van Gemert-Pijnen, J.E.W.C., et al., A holistic framework to improve the uptake and impact of eHealth technologies. Journal of medical Internet research, 2011. 13(4). 



\section{Chapter 7}

General Discussion 


\section{A brief look back}

The aim of this thesis was to gain more understanding of the potential added value of personal health records (PHRs) in healthcare, why PHRs can have this added value, and why PHRs may not have the expected impact on self-management support of patients and the daily working routines of caregivers. In Part 1 of this thesis, we focused on technology usage as a proxy for implementation and the ambiguity around the concept of adherence. These chapters have taught us that adherence to a technology is an important outcome in eHealth evaluation, but how adherence to different technologies should be defined often remains underexposed. Furthermore, log data is of great importance for determining adherence and to gain input on how to improve the persuasiveness and efficiency of eHealth technologies.

In Part 2 of this thesis, we used the knowledge obtained from Part 1 to evaluate the relation between the user, the technology and the influence of the context on the actual implementation of e-Vita. Here we learned that in the e-Vita study, the technology, the users and the context are three interrelated concepts that influenced the actual implementation of the PHR. These insights are made clear by combining quantitative and qualitative data in a mixed methods approach that highlighted the implementation process on different levels. The different studies not only served as a more traditional backward evaluation, but also as an forward evaluation to gain insight into the possibilities to improve the PHR and its implementation in the future.

In the next section, we will discuss the main findings of our studies. Thereafter, we will discuss the lessons learned and with that, we will come to directions for practice and future research and a conclusion.

\section{Main findings}

\section{Adherence and intended use}

Chapter 2 described a systematic review to clarify the concept of adherence and to find a concise way to operationalize adherence to eHealth technologies. By defining the intended use, a norm can be set to value the actual use of that technology. Our aim was to use the results of this review for formulating intended use of eHealth technologies such as PHRs. The review showed that it is often assumed that more use of a technology is always better. However, the characteristics of the technology, the (individual goals of) the users, and the context in which the technology is used, should be leading when defining the intended use. Despite these directions, it is still complicated to find an empirical or conceptual basis for the operationalization of the intended use of eHealth technologies. Therefore, only limited directions for formulating the intended use to PHRs could be derived [1]. 
We used the results of Chapter 2 for preliminary analyses to find predictors for (nonladherence. In accordance to Nijland et al., who defined the intended use for a comparable eHealth technology [2], we first used the log data of all e-Vita users to define high and low active users. However, these analyses did not yield reliable results with sufficient power. Therefore, we aimed to specify the intended use of the PHR based on the context in which it was used. As a basis, we used the pathway for chronic care incorporating the main services of a PHR that was the result of the focus groups in Chapter 6 . In this view, a user was adherent when health values were monitored at least once in every three months (before the periodic consultation with the caregiver), or when at least one health-related goal was added in the first year of use. However, these preliminary analyses showed very skewed distributions with only a few adherent users. In both preliminary analyses, the volume and the variety of the data was not sufficient for predicting (non-)adherence. Explanations for the skewed distributions, the lack of reliable results, and the consequences for our research are discussed below.

\section{The potentials of log data}

Chapter 3 described a log data protocol to show the potential of log data in eHealth evaluations [3], such as predicting adherence (see the previous section); creating user and usage profiles (e.g., [4, 5]); and predicting usage behaviour (e.g., [6]). An important precondition for these analyses however, is to have sufficient data of sufficient quality available. Unfortunately, due to implementation issues as reported in Chapters 5 and 6, the log data evaluations showed that the log data of the three PHRs was not only insufficient to predict adherence and dropouts, but we were also unable to create usage and user profiles, and to identify effective usage patterns by linking the log data to the outcomes of the e-Vita T2DM [7], CHF [8], and COPD [9] trials. The studies in Chapter 6 showed that the PHRs were hardly used due to a mismatch between the technology, the users and the context of implementation, resulting in a relatively small database of log data [10]. We could therefore conclude that the volume and the variety of the log data was influenced by the quality of the system and its implementation.

On the other hand, the analyses of the first use of the T2DM PHR [11] and the long-term use of all three PHRs [10] showed that, even with relatively small amounts of data, it was still possible to collect valuable information on how to improve the match between the technology and the users in a certain context. Thus, collecting log data in different phases of eHealth development and implementation provided the knowledge that was needed at that moment. In our studies, it provided directions on how to improve the technology and its implementation. In this way, the log data of early use allows for timely responses to flaws in the technology and directions for further (qualitative) research. By applying such shorter and more transparent evaluation cycles that allow for interim optimizations of the technology and its implementation, such analyses fit well in the agile science approach [12]. In turn, by 
optimizing the technology for users in a specific context, a richer data set for more advanced log data analyses for summative evaluations (predicting usage behaviour, creating user and usage profiles) can be obtained.

\section{The implementation of e-Vita}

\section{The intended use of e-Vita}

The systematic review in Chapter 2 showed the importance of using an empirical or conceptual substantiation of intended use to a technology, for example via an operationalization that reflects the goal of the technology or its working mechanisms. Although the review was initially conducted to improve adherence research, Chapters 5 and 6 showed that considering the intended use of a technology is important in practice as well.

For the T2DM and COPD PHRs, no specific directions for the intended use of the technology were provided to the end-users. The interviews among caregivers showed that this lack of practical guidelines for deploying the technology into daily care routines has led to several issues regarding the implementation of the $\operatorname{PHR}[10,13]$. First, the participating caregivers perceived the training regarding the intended use and the actual deployment of the different services of the PHRs in daily working routines as insufficient. After the training, caregivers were still mostly ignorant on what was expected from them (except for administering questionnaires at certain points in time) and how to deploy the different services of the PHRs in such a way that they add value to the current protocols, their own working routines, and the development of self-management skills of their patients. As a result, caregivers found it hard to instruct their patients in using the PHR and they established workarounds for the different services of the PHR. For example, printing lab results from their own EHR instead of referring patients to the PHR (T2DM) or contacting a patient via telephone, instead of checking patient reported information via the PHR (CHF). The relative advantage (the benefit of the PHR compared to current practice), which is seen as an important determinant for the diffusion of potential innovations $[14,15]$ is thus perceived as low. Moreover, the interviews in Chapters 5 and 6 showed that a stimulus to establish new working routines requires incentives (e.g., guidance, time, money, or other benefits) that were missing in the project. A previous systematic review regarding the barriers for PHR adoption showed that this might have caused a resistance to change [16].

Several systematic reviews emphasize the role of caregivers in the process of PHR adoption by patients. Archer et al. for example, found that low caregiver awareness and preparedness reduce the chance of a successful adoption of PHRs [16], and Nazi emphasizes the importance of decent training and support in this process [17]. The results from our studies showed that this training should go beyond just providing information and should provide the opportunity to try the technology, e.g., by using scenarios or simulations. 
The involvement of all end-users (both patients and caregivers) in determining the intended use that adds value to self-care and the integration of the technology in daily (working) routines can create a basis for such training [16]. This hypothesis is supported by the findings in Chapter 6 , showing that only a relatively small investment of involving 13 caregivers in two focus group meetings of $1,5 \mathrm{~h}$ each resulted in a draft of an updated care pathway that incorporates the main services of PHRs (monitoring, coaching, education, logistic support). By incorporating these services into the current clinical pathways, this draft has the potential to support the shift from the more clinical working routines for institutionalized and episodic care for chronic patients as passive recipients, to integrated care pathways that provide continuous chronic care and support patients to be actively involved [18].

During the subsequent operationalization phase, it might then be of added value to increase the observability and the trialability of the PHR [15], for example by appointing ambassadors, peers who use the PHR in daily routines. By experiencing the technology (e.g., during training) and by seeing how someone else is using the technology and takes advantage of it, a larger group of potential end-users can acknowledge that the technology is safe and beneficial, which can increase the chances for a larger-scale adoption [14]. However, these chance will only increase when the potential added value of the new technology is already evident in the first stages of the developmental cycle $[16,19]$. The added value of this approach will be discussed below.

\section{Usability and the first impression}

According to previous studies, the first impression of eHealth technologies [2,5] and the usability [16] are key to the subsequent adoption and use. Our results show that there is only one chance for a first impression and that a negative first impression can negatively impact the actual use of the PHR.

In Chapter 6, usability issues (e.g., problems with logging in and unclear terminology) were commonly reported by all end-users. Furthermore, the log data analyses in Chapter 4 showed that when users visited the education service as a first step after the first login, almost all users ended that session after opening one education topic [11]. Moreover, a large proportion of this group did not return for a second session. It could be that the users were overwhelmed by the first impression of the education service, and discouraged to continue using the PHR. Another explanation could be that users got astray by the referral to an external website via a pop-up, although this does not completely explain the lacking longterm use. Furthermore, most users were likely to either log out after visiting one service, or after visiting five or more services. This indicates that the first impression of the PHR might not be attractive, but when users overcame this first impression, they made an effort to explore the rest of the PHR by visiting the other services as well. This situation is probably 
recognizable for every smartphone user: a new app is installed in a snap, but removed just as fast when the first impression does not match the expectations.

Up to now, log data analyses are mainly used to gain insight into the uptake of a technology in the summative evaluation phase. To our concern however, together with research of Kelders et al. [20], the log data analyses in our studies are one of the first that demonstrate their added value during the early operationalization, in order to improve the system design and to identify prompts for motivating and engaging users. In this way, the match between the system and its users can be improved, and the long-term adherence has the potential to increase.

To summarize, understanding the interplay between the technology, its users and the context of implementation were of great importance when interpreting the limited results of the e-Vita project. Furthermore, to increase the added value and to facilitate the implementation, it is essential to not just start the conversation with end-users in the early stages of eHealth development, but to keep this conversation going (e.g., via interviews, focus groups, usability tests) throughout the entire process of development, operationalization, and summative evaluation. A mixed methods approach and short and iterative cycles are of added value in providing explanations for the found effects that could not be revealed by solely focusing on the outcomes of experimental trials.

\section{Hierarchy of needs}

Another striking result from our studies was that caregivers and the potential end-users hardly mentioned privacy issues as barriers for using the PHRs. These barriers are, especially among non-users, mentioned in a large number of previous studies (e.g., [16, 21]). However, although we did not explicitly ask how participants perceived their own privacy (potential end-users) or the privacy of their patients (caregivers) on the PHR, these issues were certainly not on top of their mind in this research project. Our results therefore imply that there could be a hierarchy (comparable to Maslow's Hierarchy of Needs [22]), where the need for user friendly technologies that meet the demands of the users and fit within the context of implementation, should be fulfilled before being able to progress to the higher levels of safety concerns. However, no support for this hypothesis could be found in current literature.

\section{Lessons learned}

No research comes without lessons learned. Therefore, we will now share the most important lessons from this research project.

\section{Lesson 1: Implementation is not a post-design step}

In this thesis, the principles of the CeHRes roadmap [19, 23] were used as the basis for our research. The roadmap states that eHealth development is a holistic, participatory and 
iterative process that is intertwined with the implementation into daily (care) routines. In this view, implementation starts already in the first phases of eHealth development, when gaining insight into the context of the implementation and the needs and values of the stakeholders.

e-Vita was developed on the basis of brainstorm sessions with patients and caregivers to gain insight into their experiences with living with a chronic disease (patients) or providing care for the chronically ill (caregivers). Based on this information, requirements for the PHR were communicated to the technical developers of the PHR, who translated the requirements into a first version of the PHR for T2DM and CHF. The subsequent iterative development cycles that were used within this process mainly consisted of expert-based usability tests among the researchers and other project participants. Although such tests have the potential to identify technical errors or inconsistencies within the technology, they also tend to invite participants to think for the users. In other words, such expert-based usability tests might disregard the fit with the context in which the technology was deployed and the needs and wishes of the actual end-users that could have come forward in user-based usability tests among patients and caregivers [24].

Only in the operationalization phase, when the PHR already had been operationalized for a while, usability evaluations revealed that patients experienced difficulties in determining what is expected from them when using the technology and finding the services or information they wanted. Furthermore, the used terminology was perceived as unclear (Chapter 6). In addition, the interviews among caregivers provided directions for improving the implementation of the PHR, and the log data analyses of the first use of e-Vita T2DM provided prompts for improving the first impression of the technology. However, due to the nature of the ongoing trials to evaluate the effectiveness of the PHRs, it was no longer possible to make adjustments to the technology.

From the results of this study it could thus be concluded that it is of utmost importance to involve all end-users in all stages of eHealth development and to value the technology through the eyes of these different stakeholders. E.g., what data can support the development of self-management skills by patients, and how should these data be visualized? What terminology should be used? And at the same time, how should the data as collected by patients be presented to caregivers in order to support medical decision making? How can patients be stimulated to provide the necessary data anyway? Taking into account these different views has the potential to increase the added value for all stakeholders and to facilitate the actual operationalization of the technology. In that way, the problems as described above would have been identified in earlier stages where they could have been accounted for. 
Short and iterative development and formative ('agile') evaluation cycles among all stakeholders are thus crucial to allow for timely adjustments [12, 19] and to identify problems already in early stages of development (or as Glasgow states: to 'fail rapidly' [25]). For example, the usability problems as described in Chapter 6 (e.g., unclear terminology and visualizations, unclickable 'buttons') could easily have been solved before the actual operationalization phase, which could have made the PHR more attractive and clear for the end-users.

\section{Lesson 2: A PHR will not implement automatically once it is offered to the end-users.}

As stated in the introduction, eHealth development is not about creating and evaluating just 'a thing' or a tool, but it is rather about creating and evaluating an infrastructure for knowledge dissemination, communication and the organization of care [26]. However, one of the main conclusions of this thesis should be that a large-scale penetration of this approach was lagging in the current project.

Unfortunately, these results are not new: limited use is a common problem in PHR evaluations [27]. Several recent systematic reviews focusing on the implementation of complex (eHealth) interventions and PHRs stress that the (perceived) fit of eHealth technologies with the current working routines and the interoperability with other systems influence a successful implementation $[15,27,28]$. This was also seen in a study of Wentzel et al., who found that constantly keeping an eye on the context of implementation when developing eHealth, resulted in a better support of caregivers in performing their daily tasks [29]. Or, as Wells et al. state: "the key to clinician acceptance is making their work easier" [30]. In turn, an engaged caregiver is often seen as the key to patient engagement in PHR adoption [31-33]. As stated before, a decent training and support of all end-users in deploying the system is thus necessary. On the other hand, when the first impression is moderate and the added value is thus lacking (as seen in our current research), it will still hardly or not be used at all [34]. Developing technology with added value for all end-users thus requires a holistic and iterative approach of eHealth development, implementation and evaluation $[27,35,36]$.

\section{Lesson 3: A holistic evaluation approach is crucial in gaining insight into the success of an eHealth technology.}

Up to now, experimental trials are demanded by health insurance companies to determine whether the use of a technology will be reimbursed. However, such experimental trials do not provide the complete picture regarding the effectiveness of the technology. eHealth development and implementation is a multi-level and complex process, that requires a holistic evaluation approach taking into account how a technology is of added value for 
certain users in a certain context $[15,27,28]$. To be able to better understand why the results of the PHR so far were disappointing [37-39], we used a mixed methods approach that combined multiple levels of data collection [40]. These insights on the usage of the PHRs and the experiences of the end-users could not have been obtained by solely focusing on the effectiveness of the technology in an experimental trial.

Our findings support the recently published recommendation of the Dutch Council for Public Health and Society (in Dutch: Raad voor Volksgezondheid en Samenleving) that pleads the case for a shift from evidence-based medicine to context-based medicine [41]. They state that evidence is obviously an important source of information when evaluating an intervention, but it is influenced by the context of the patient, the care setting, and the connection between these two as well [42]. The context and the implementation process should therefore also be subject of the evaluation, since they determine the validity of the evidence as obtained in trials. The council therefore makes a plea for combining evidence from trials with other sources of quantitative and qualitative data to determine the effectiveness and added value of interventions [41].

In the future, the recommendations of the council and evaluations as described in this thesis, might play an important role in the abovementioned shift from evidence-based to contextbased 'eHealth practice' and reimbursements for the implementation of eHealth technologies. These reimbursements might stimulate the healthcare practice to integrate eHealth technologies into daily care routines and have therefore the potential to boost the uptake of PHRs as well [34].

For eHealth technologies, this recommendation implies short and iterative formative evaluation cycles that provide insights into the potential success or failure of a technology already in early stages of eHealth development. In this approach, a technology will only be operationalized on a larger scale when no new usability issues were identified and when e.g. focus groups indicated that the technology fits the needs of the end-users and their context. After the operationalization, log data can provide continuous insights into the actual uptake of the technology and can provide input for e.g., interviews, focus groups, and questionnaires to gain additional information about the exact working mechanisms of a technology and why the use of the technology may or may not lead to the desired effects.

\section{Strengths and Limitations}

Just as in any other research project, the research in this thesis has strengths and limitations. An important limitation is that, due to privacy issues, we were unable to involve the actual patient-users of e-Vita in qualitative studies to gain insight into their experiences with using the PHR. Therefore, we did not obtain insight into the influence of specific patient-related barriers (e.g., self-efficacy, individual capabilities $[21,34]$ ) on the actual use of the PHR and 
were not able to test our hypothesis regarding privacy and confidentiality issues. However, since saturation of the data had been reached, we do believe that the interviews among caregivers provided a decent impression of the implementation process of the PHR and the interactions between patients and caregivers. Moreover, we were able to conduct usability tests among potential end-users, who were patients with the same chronic conditions and/or contemporaries of the actual users, and thus belonging to the same target group as the actual patient users. We therefore believe that these participants provided a rather good impression of the needs and wishes of chronically ill people and the usability issues that the actual users might have experienced.

As described in the research questions in the General Introduction, we planned to conduct log data analyses to predict adherence and to create user and usage profiles. However, the body of log data that was collected during the study period was not rich enough to conduct these analyses. Nevertheless, we do feel that we demonstrated that it is even possible to obtain valuable insights into the actual use of a technology by using the more 'simple' descriptive statistics.

On the other hand, it would have been a great opportunity to link the log data to the outcome measures of the different trials, to investigate how the use of the technology can lead to certain outcomes. However, no significant outcomes were found in the trials regarding the effectiveness of the PHR, and the log data set was not rich enough to perform such analyses.

The use of log data was an important strength in our study. As we described in Chapter 3, a main advantage of log data is that it is always available and easy to collect, without requiring any extra effort from the participants. A common problem in (eHealth) research is that participants often find it time consuming and labour intensive to complete questionnaires at different time points or to participate in qualitative research, often resulting in high dropout rates from the study. However, this result does not necessarily mean that these participants stopped using the technology as well. By mixing the objective quantitative log data with the other subjective qualitative data sources, we were no longer dependent on recalled behaviour and having a majority of the participants completing questionnaires or participating in qualitative studies. By that, we were able to gain a good understanding of the implementation process of the PHR in Chapter 6.

\section{Considerations for Future Research}

\section{Unambiguous measures of adherence}

The research in this thesis contributed to answering the question why PHRs may or may not have the expected impact. However, additional research is needed to understand how PHRs can make a difference in the future. We concluded Chapter 2 of this thesis with a proposition 
for measuring adherence to eHealth technologies. This approach simplifies the comparison

of adherence to PHRs with a different intended use due to differences in characteristics (e.g., the services that are offered, persuasiveness, user-friendliness, context). This approach will be of added value for both PHR developers and researchers, since it allows for a better understanding of how different services of PHRs support patients in developing selfmanagement skills and how to support user adherence in relation to the desired outcomes.

\section{Improving the implementation to collect data that matters}

As described in Chapter 3, log data can, among other things, be used to obtain real-time insights into the usage patterns of long-term users and who the drop-outs are. Based on this information, user profiles can be identified that allow for personalization of the technology. Several studies have demonstrated that individuals respond differently to the same strategies for behaviour change (e.g., persuasive strategies) [43], or that the use of certain features increases adherence to the technology (e.g., [4]) indicating that adapting the technology to individual users (personalization) might increase the persuasiveness of a technology and thus its long-term use and effectiveness [44].

In the same chapter however, we described that for such predictions, the log data files should be of sufficient quality. In other words, when the data contains the information of 100 usage session, this data should rather contain information of 25 sessions of 4 different users, instead of the information of 100 users having 1 usage session each. However, Chapter 6 revealed that the actual use of the PHR was lagging, and preliminary analyses to predict adherence and dropping-out showed that the log data was not rich enough for making sound predictions. Therefore, we were not able to answer all research questions. As previously explained, this lack of data is largely due to the limited implementation of the PHR. However, user and usage profiles and predictors for adherence and dropping-out can still be of added value to increase the match between the user and the technology in certain contexts, but being able to collect such data requires PHRs that are actually used on the long-term.

\section{Big data for data-driven coaching}

The data that is being collected via PHRs provides great opportunities for data-driven coaching of chronically ill. Large amounts of valid, real-time data generated by technologies (e.g., log data, monitoring data) allow for advancing models and theories of behaviour change to support individuals in changing their behaviour and that enable the personalization of technologies $[36,45]$. However, in order to do so, a sufficient amount of data with sufficient variety is needed. Furthermore, this data cannot be returned to the endusers without any consideration, but it should rather be brought back in ways that are meaningful to the patients and supportive for caregivers without being just overwhelming surveillance data [25]. In order to do so, needs assessments and other methods for testing mock-ups among potential end-users can provide valuable insights in how caregivers use 
health-related data in decision making and in turn, this information can be used to improve eHealth technologies.

\section{To conclude}

This thesis showed the inseparable relation between the user, the technology and the context in the implementation and evaluation of eHealth technology. Understanding the interplay between these three concepts provided insight into why the implementation of the personal health record e-Vita did not lead to the desired effects. To conclude, a personal health records will not implement automatically once it is offered to the end-users, and a holistic and iterative approach of eHealth development, implementation and evaluation is crucial in the success of an eHealth technology.

In this view, it is of utmost importance to involve all end-users in all stages of eHealth development to increase the added value and to facilitate the implementation. Short and iterative development cycles are crucial for identifying problems in early stages of development and to increase the chances for a successful implementation. When evaluating the implementation process, a mixed methods approach is of added value in providing explanations for the found effects that could not be revealed by solely focusing on the effectiveness of the technology in an experimental trial.

With this research, we aimed to contribute to the expansion of the CeHRes roadmap with indepth knowledge to combine methods for formative and summative evaluations. In the bigger picture, we aimed to contribute to the current body of research regarding using advanced approaches to open black box of eHealth. 


\section{References}

1. Sieverink, F., S. Kelders, and J. Van Gemert-Pijnen, When does usage become adherence? A systematic review to clarify the concept of adherence to eHealth technology. J Med Internet Res (forthcoming). doi:10.2196/jmir.8578

2. Nijland, N., et al., Factors influencing the use of a Web-based application for supporting the selfcare of patients with type 2 diabetes: a longitudinal study. Journal of Medical Internet Research, 2011. 13(3).

3. Sieverink, F., et al., Opening the Black Box of Electronic Health: Collecting, Analyzing, and Interpreting Log Data. JMIR Res Protoc, 2017. 6(8): p. e156.

4. Freyne, J., et al. Factors associated with persistent participation in an online diet intervention. in CHI'12 Extended Abstracts on Human Factors in Computing Systems. 2012. ACM.

5. Kelders, S.M., E.T. Bohlmeijer, and J.E.W.C. Van Gemert-Pijnen, Participants, usage, and use patterns of a web-based intervention for the prevention of depression within a randomized controlled trial. Journal of Medical Internet Research, 2013. 15(8).

6. Räisänen, T., H. Oinas-Kukkonen, and S. Pahnila, Finding Kairos in Quitting Smoking: Smokers' Perceptions of Warning Pictures, in Persuasive Technology: Third International Conference, PERSUASIVE 2008, Oulu, Finland, June 4-6, 2008. Proceedings, H. Oinas-Kukkonen, et al., Editors. 2008, Springer Berlin Heidelberg: Berlin, Heidelberg. p. 254-257.

7. Roelofsen, Y., et al., Design of the e-Vita diabetes mellitus study: Effects and use of an interactive online care platform in patients with type 2 diabetes (e-VitaDM-1/ZODIAC-40). Acta Veterinaria Scandinavica, 2014: p. 22.

8. Wagenaar, K.P., et al., Effectiveness of an interactive platform, and the ESC/HFA heartfailurematters.org website in patients with heart failure: design of the multicentre randomized e-Vita heart failure trial. European Journal of Heart Failure, 2015. 17(12): p. 1310-1316.

9. Talboom-Kamp, E.P.W.A., et al., e-Vita: design of an innovative approach to COPD disease management in primary care through eHealth application. BMC Pulmonary Medicine, 2016. 16(1): p. 121.

10. Sieverink, F., et al., Evaluating the implementation of a Personal Health Record for chronic primary and secondary care: A mixed methods approach. Submitted.

11. Sieverink, F., et al., The Added Value of Log File Analyses of the Use of a Personal Health Record for Patients With Type 2 Diabetes Mellitus: Preliminary Results. Journal of Diabetes Science and Technology, 2014. 8(2): p. 247-255.

12. Hekler, E.B., et al., Agile science: creating useful products for behavior change in the real world. Translational Behavioral Medicine, 2016. 6(2): p. 317-328.

13. Sieverink, F., et al. The Uptake and Impact of a Personal Health Record for Patients with Type 2 Diabetes Mellitus in Primary Care: A Research Protocol for a Backward and Forward Evaluation. in eTELEMED 2014, The Sixth International Conference on eHealth, Telemedicine, and Social Medicine. 2014.

14. Cain, M. and R. Mittman, Diffusion of innovation in health care. 2002: California Healthcare Foundation Oakland CA.

15. Ross, J., et al., Factors that influence the implementation of e-health: a systematic review of systematic reviews (an update). Implementation Science, 2016. 11(1): p. 146. 
16. Archer, N., et al., Personal health records: A scoping review. Journal of the American Medical Informatics Association, 2011. 18(4): p. 515-522.

17. Nazi, K.M., The Personal Health Record Paradox: Health Care Professionals' Perspectives and the Information Ecology of Personal Health Record Systems in Organizational and Clinical Settings. J Med Internet Res, 2013. 15(4): p. e70.

18. Organization, W.H., Innovative care for chronic conditions: building blocks for actions: global report. 2002.

19. Van Gemert-Pijnen, J.E.W.C., et al., A holistic framework to improve the uptake and impact of eHealth technologies. Journal of medical Internet research, 2011. 13(4).

20. Kelders, S.M., Understanding adherence to web-based interventions. 2012: Enschede. p. 248.

21. Showell, C., Barriers to the use of personal health records by patients: a structured review. PeerJ, 2017. 5: p. e3268.

22. Maslow, A.H., R. Frager, and J. Fadiman, Motivation and personality. Vol. 2. 1970: Harper \& Row New York.

23. Van Gemert-Pijnen, J.E.W.C., O. Peters, and H.C. Ossebaard, Improving eHealth. 2013, The Hague, the Netherlands: Eleven International Publishing.

24. Jaspers, M.W.M., A comparison of usability methods for testing interactive health technologies: Methodological aspects and empirical evidence. International Journal of Medical Informatics, 2009. 78(5): p. 340-353.

25. Glasgow, R.E., S.M. Phillips, and M.A. Sanchez, Implementation science approaches for integrating eHealth research into practice and policy. International Journal of Medical Informatics, 2014. 83(7): p. e1-e11.

26. Eysenbach, G., What is e-health? J Med Internet Res, 2001. 3(2): p. e20.

27. Otte-Trojel, T., et al., What do we know about developing patient portals? a systematic literature review. Journal of the American Medical Informatics Association, 2016. 23(e1): p. e162-e168.

28. Lau, R., et al., Achieving change in primary care-causes of the evidence to practice gap: systematic reviews of reviews. Implementation Science, 2016. 11(1): p. 40.

29. Wentzel, J., et al., Antibiotic information application offers nurses quick support. American Journal of Infection Control, 2016. 44(6): p. 677-684.

30. Wells, S., et al., Organizational strategies for promoting patient and provider uptake of personal health records. Journal of the American Medical Informatics Association, 2015. 22(1): p. 213-222.

31. Krist, A.H., et al., Engaging Primary Care Patients to Use a Patient-Centered Personal Health Record. The Annals of Family Medicine, 2014. 12(5): p. 418-426.

32. Amante, D.J., et al., A Systematic Review of Electronic Portal Usage Among Patients with Diabetes. Diabetes Technology \& Therapeutics, 2014. 16(11): p. 784-793.

33. Agarwal, R., et al., If We Offer it, Will They Accept? Factors Affecting Patient Use Intentions of Personal Health Records and Secure Messaging. J Med Internet Res, 2013. 15(2): p. e43.

34. Damschroder, L., et al., Fostering implementation of health services research findings into practice: a consolidated framework for advancing implementation science. Implementation Science, 2009. 4(1): p. 50.

35. Wells, S., et al., Personal Health Records for Patients with Chronic Disease: A Major Opportunity. Applied Clinical Informatics, 2014. 5(2): p. 416-429. 
36. Michie, S., et al., Developing and Evaluating Digital Interventions to Promote Behavior Change in Health and Health Care: Recommendations Resulting From an International Workshop. J Med Internet Res, 2017. 19(6): p. e232.

37. Talboom-Kamp, E.P., et al., The Effect of Integration of Self-Management Web Platforms on Health Status in Chronic Obstructive Pulmonary Disease Management in Primary Care (e-Vita Study): Interrupted Time Series Design. J Med Internet Res, 2017. 19(8): p. e291.

38. van Vugt, M., et al., Uptake and Effects of the e-Vita Personal Health Record with Self-Management Support and Coaching, for Type 2 Diabetes Patients Treated in Primary Care. Journal of Diabetes Research, 2016. 2016: p. 9.

39. Wagenaar, K., E-health in heart failure. 2017, University Utrecht.

40. Creswell, J.W., Research design: Qualitative, quantitative, and mixed methods approaches. 2013: Sage publications.

41. Samenleving, R.v.V.e., Zonder context geen bewijs. Over de illusie van evidence-based practice in de zorg. 2017: The Hague, the Netherlands.

42. Nazi, K.M., The personal health record paradox: Health care professionals' perspectives and the information ecology of personal health record systems in organizational and clinical settings. Journal of Medical Internet Research, 2013. 15(4).

43. Kaptein, M. and D. Eckles, Heterogeneity in the Effects of Online Persuasion. Journal of Interactive Marketing, 2012. 26(3): p. 176-188.

44. Kaptein, M., et al., Personalizing persuasive technologies: Explicit and implicit personalization using persuasion profiles. International Journal of Human-Computer Studies, 2015. 77: p. 38-51.

45. Patrick, K., et al., The Pace of Technologic Change. American Journal of Preventive Medicine, 2016. 51(5): p. 816-824. 

The Personal Health Record e-Vita 
e-Vita Diabetes

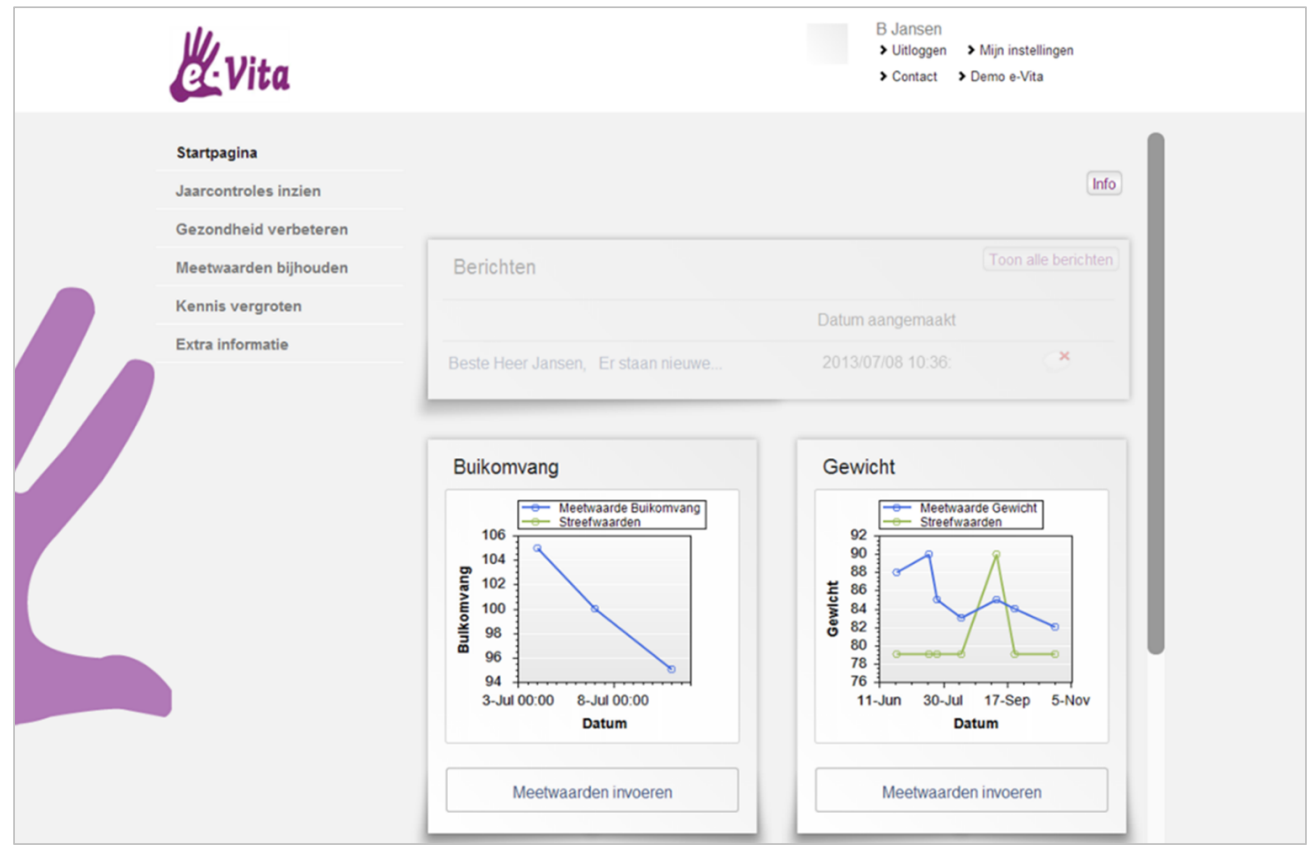

The homepage of e-Vita Diabetes. Users were able to access all features via the menu structure on the left.

\begin{tabular}{|c|c|c|c|c|}
\hline \multicolumn{5}{|l|}{ Startpagina } \\
\hline Jaarcontroles inzien & & & & Info \\
\hline Gezondheid verbeteren & & & & \\
\hline Meetwaarden bijhouden & \multicolumn{2}{|r|}{ Dit wil ik bereiken... (mijn wensen) } & Nieuwe wens & Alle wensen \\
\hline \multicolumn{5}{|l|}{ Kennis vergroten } \\
\hline \multicolumn{5}{|l|}{ Extra informatie } \\
\hline & $\checkmark$ & Een dagje winkelen & & 玍Actieplan \\
\hline & $\checkmark$ & Ik wil afvallen & & 经Actieplan \\
\hline & $\checkmark$ & ik wil graag beter kunnen lopen & & 经Actieplan \\
\hline
\end{tabular}

The online coach for guidance when working on personal, health-related goals. 


\begin{tabular}{|c|c|c|c|c|c|c|}
\hline Cijfers & Grafieken & & & & & \\
\hline \multicolumn{2}{|c|}{ Meetwaarden } & & 2009 & 2010 & 2011 & 2012 \\
\hline Uitleg & $\mathrm{HbA1c}$ & $\mathrm{mmol} / \mathrm{mol}$ & & & & \\
\hline Uitleg & Bovendruk & $\mathrm{mmHg}$ & & & & \\
\hline Uitleg & Onderdruk & $\mathrm{mmHg}$ & & & & \\
\hline Uitleg & Cholesterol & $\mathrm{mmol} / \mathrm{L}$ & & & & \\
\hline Uitleg & HDL & $\mathrm{mmol} / \mathrm{L}$ & & & & \\
\hline Uitleg & LDL & $\mathrm{mmol} / \mathrm{L}$ & & & & \\
\hline Uitleg & Triglyceride & $\mathrm{mmol} / \mathrm{L}$ & & & & \\
\hline Uitlea & Cockcroft & $\mathrm{ml} / \mathrm{min}$ & & & & \\
\hline
\end{tabular}

Top: Insight in lab values, provided by the general practitioner. All values are explained via an information button.

Middle: Self-monitoring personal health values (waist circumference, weight, blood pressure, BMI)

Bottom: Education feature, partly tailored to the user.

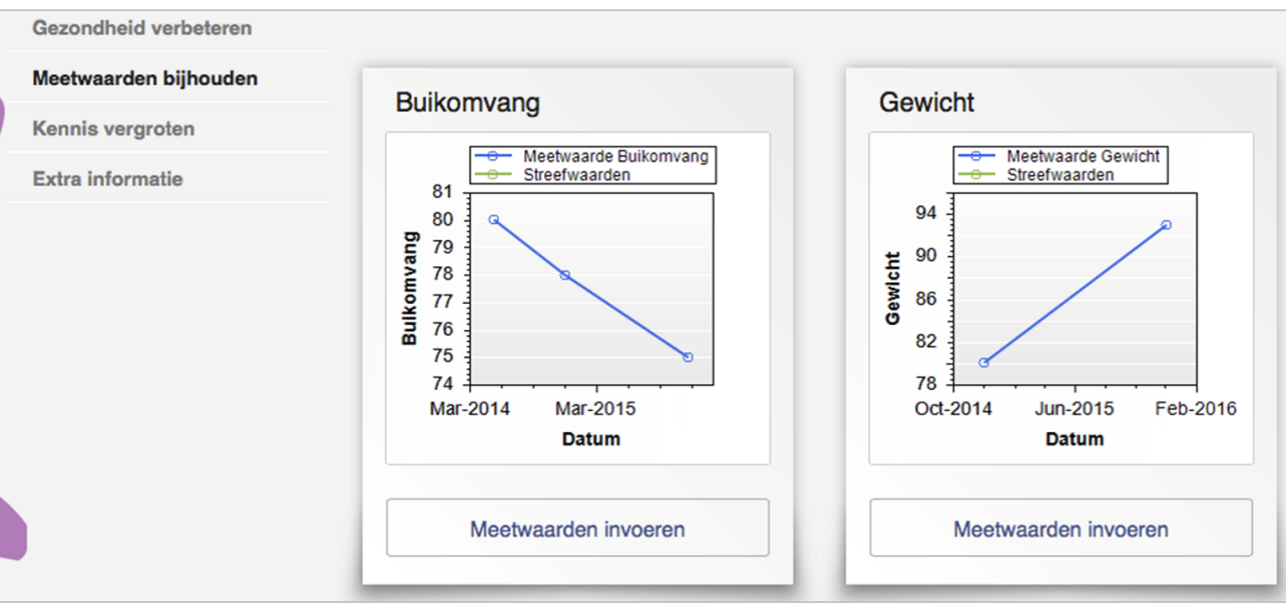

Jaarcontroles inzien

Gezondheid verbeteren

Meetwaarden bijhouden

Kennis vergroten

Extra informatie

\section{Educatie}

\begin{tabular}{|c|c|c|}
\hline$\triangleright \quad \mid \vee I$ & Algemeen & $40 \%$ \\
\hline$\triangleright \quad|\checkmark|$ & Labwaarden & $\square 10 \%$ \\
\hline 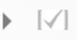 & Leefstijl & $40 \%$ \\
\hline$\Rightarrow \quad \mid \vee I$ & Complicaties & $\square 70 \%$ \\
\hline$\triangleright \quad \mid \checkmark 1$ & Medicatie & $30 \%$ \\
\hline$\rightarrow \quad|\checkmark|$ & Overige & $\longrightarrow 0 \%$ \\
\hline
\end{tabular}




\section{e-Vita COPD}

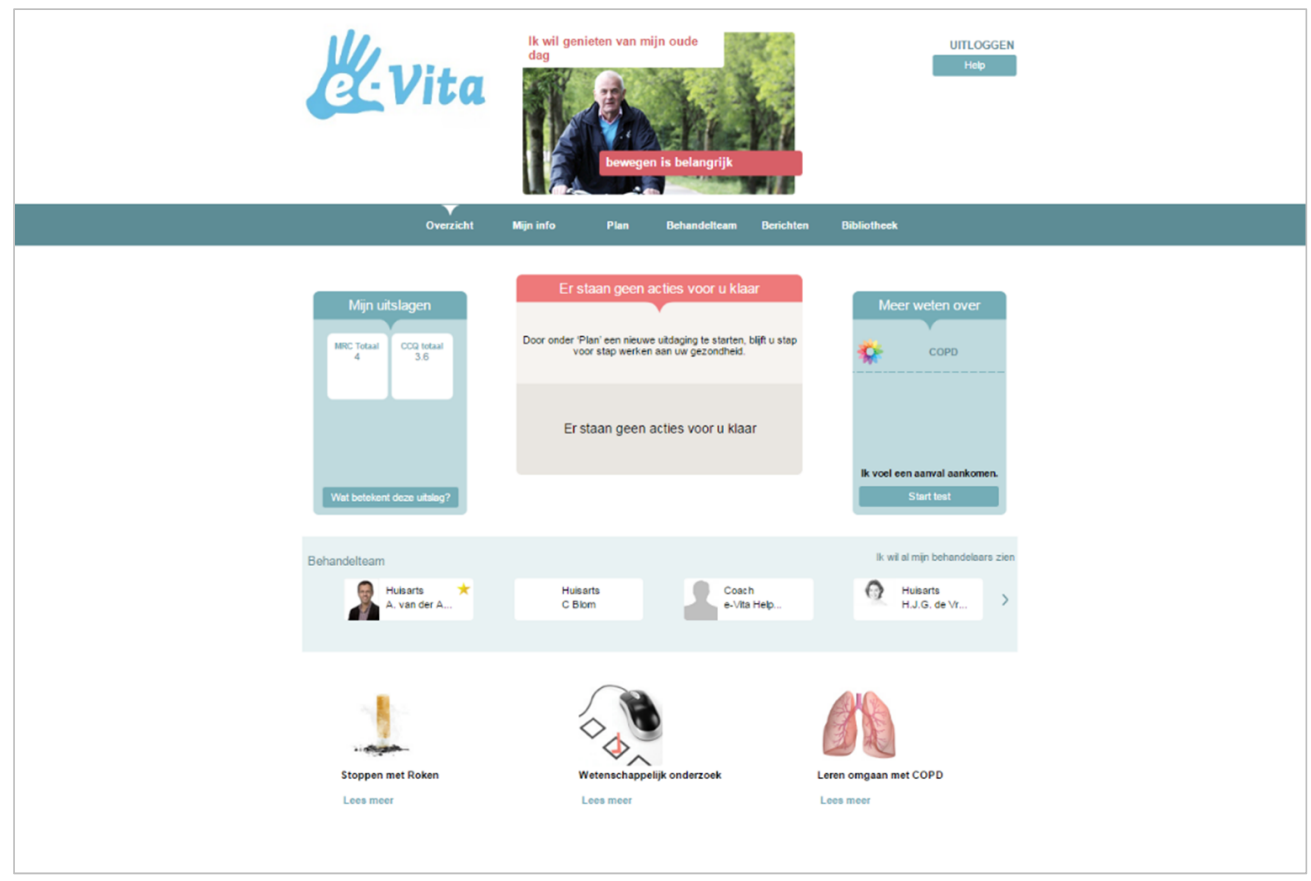

The homepage of e-Vita COPD. Users were able to access all features via the tabs on the top of the page.

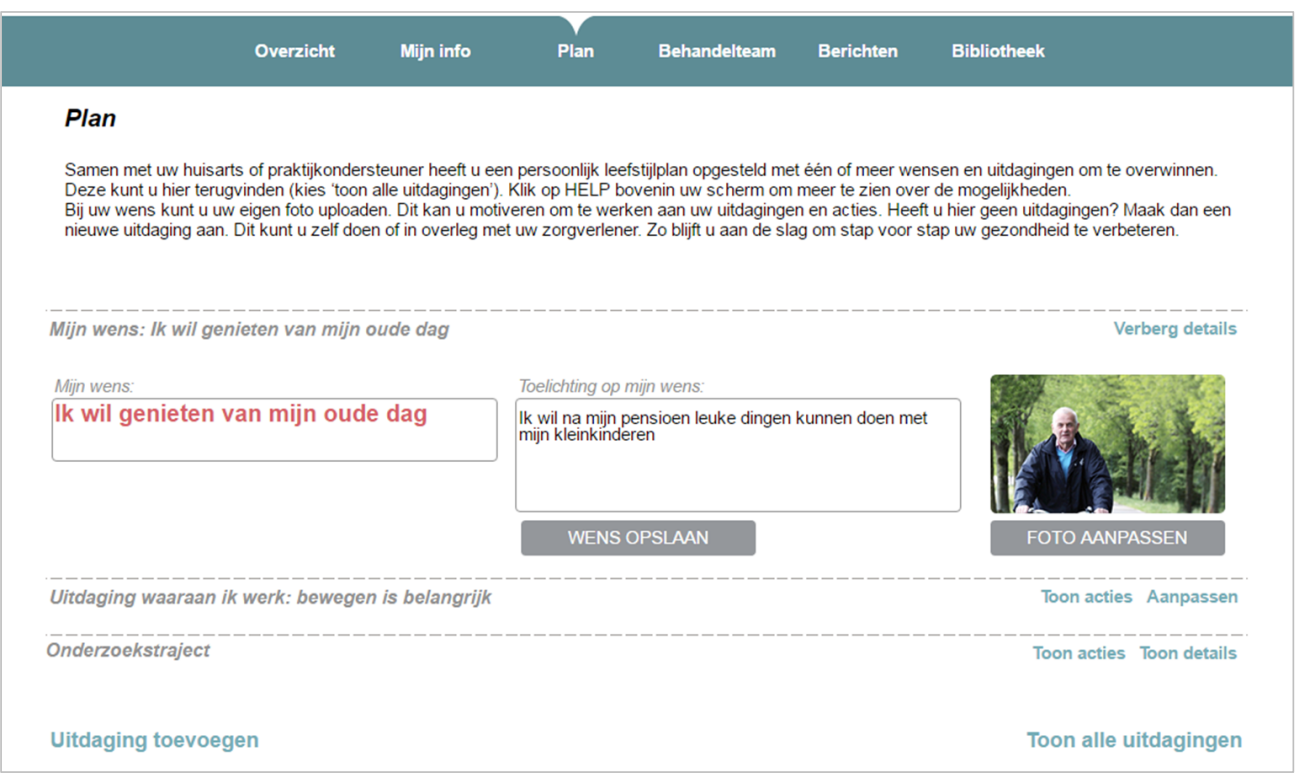

The online coach for guidance when working on personal, health-related goals. 


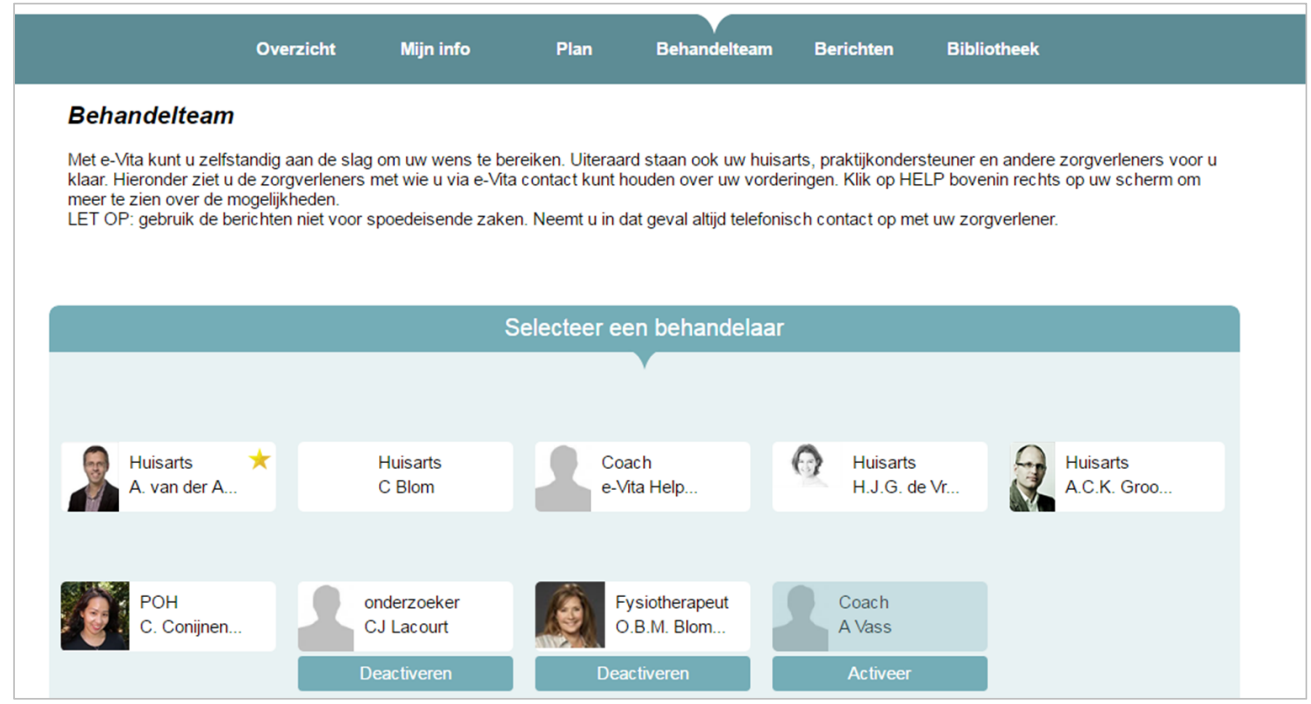

An overview of the healthcare team of the user. Here, users were able to send a message to a care provider.

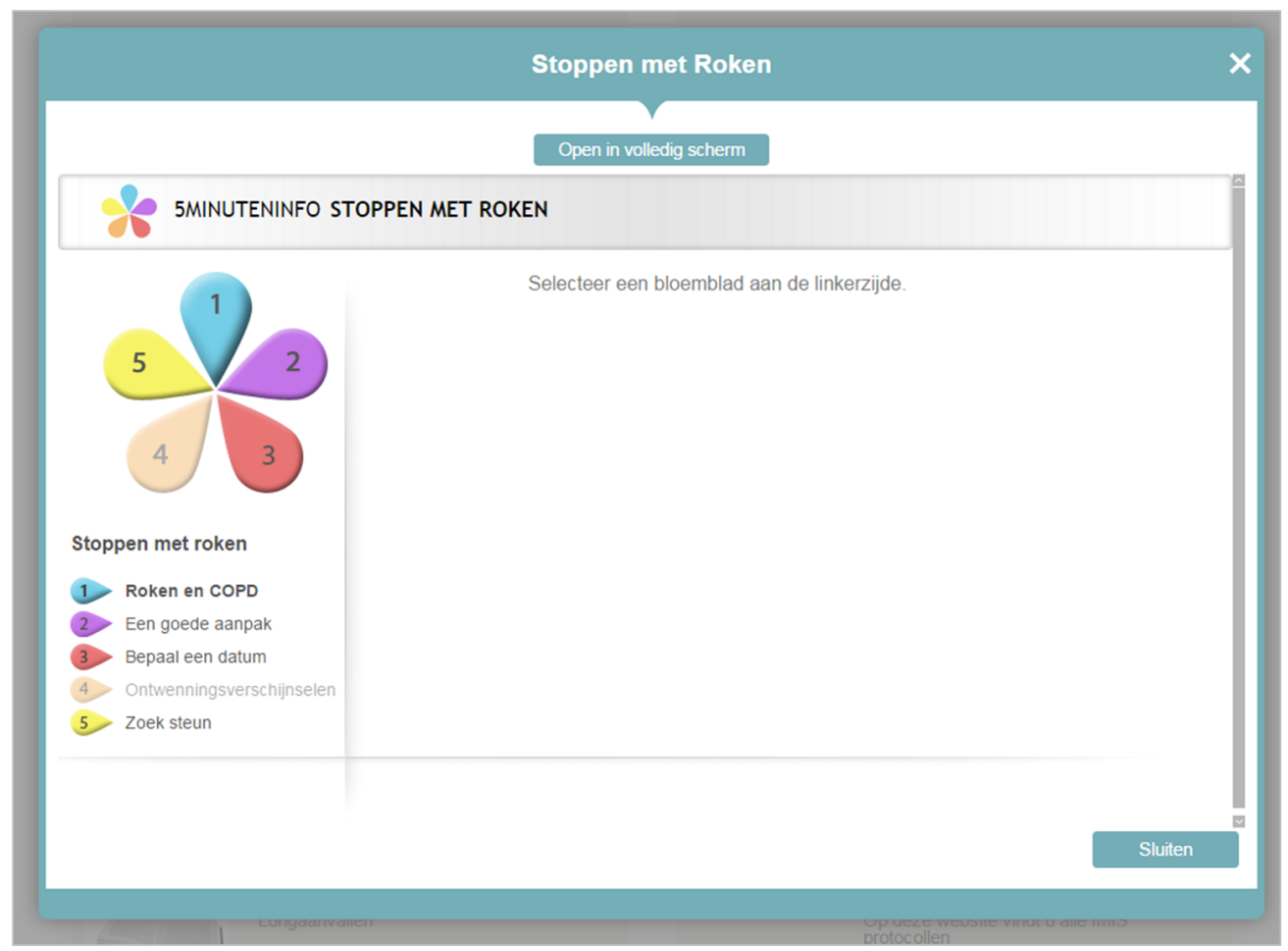

Education feature, partly tailored to the user. 


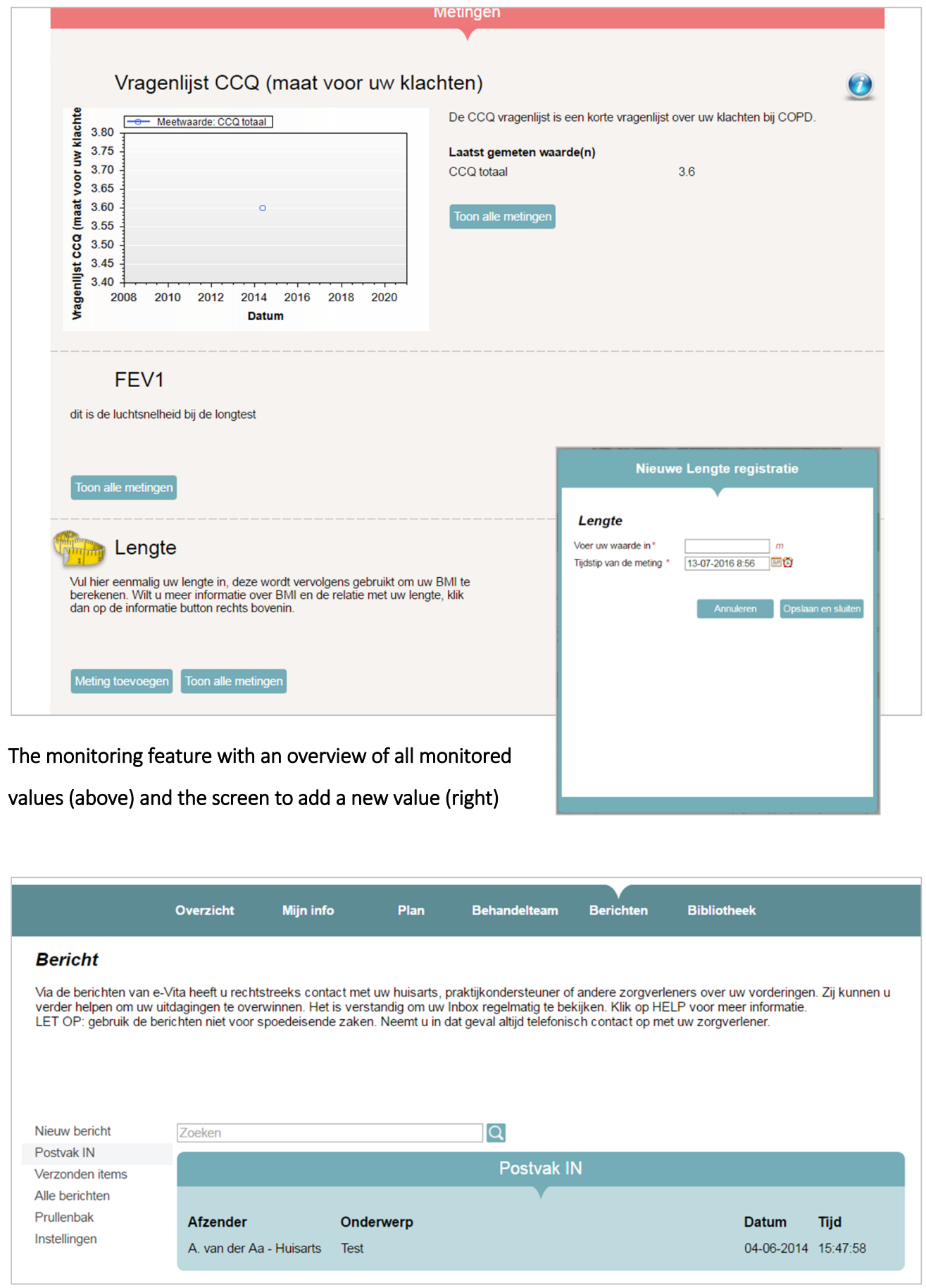

The message feature where users were able to read and send messages

172 | The Personal Health Record e-Vita 




\section{Samenvatting}

(Summary in Dutch) 
De vergrijzing en de toename van het aantal mensen met een chronische aandoening (zoals diabetes mellitus type 2 , chronisch hartfalen, of COPD) zorgen voor een toenemende druk op de gezondheidszorg. Dit heeft een aantal gevolgen. Bijvoorbeeld, door de stijgende zorgkosten vragen zorgverzekeraars steeds vaker om patiënten in de tweede lijn (bijv. in een polikliniek) terug te verwijzen naar de eerste lijn (de huisartsenpraktijk). Hierdoor zijn de zorgverleners in de huisartsenpraktijk verantwoordelijk voor een groeiend aantal taken met betrekking tot de begeleiding en behandeling van chronisch zieken, terwijl door de vergrijzing steeds minder zorgverleners beschikbaar zijn om deze verschuiving op te vangen. Daarnaast is de gezondheidszorg in de afgelopen decennia dusdanig verbeterd dat het steeds normaler wordt om oud te worden met één of meerdere chronische aandoeningen. In dit licht is gezondheid niet langer de afwezigheid van ziekte of zwakte (zoals gedefinieerd door de WHO), maar juist het 'vermogen om je aan te kunnen passen en je eigen regie te voeren' (Huber, 2011). Duurzame oplossingen zijn nodig om deze veranderingen in het zorglandschap op te kunnen vangen.

eHealth toepassingen, zoals personal health records (PHRs) bieden de mogelijkheid om patiënten meer te betrekken in hun eigen zorg. In het Nederlands zijn dergelijke toepassingen beter bekend als patiëntenplatformen of patiëntenportalen. Op dergelijke PHRs kunnen patiënten bijvoorbeeld hun eigen gegevens inzien en delen met hun zorgverleners, educatie krijgen over hun aandoening, werken aan hun persoonlijke gezondheidsdoelen, korte vragen stellen aan zorgverleners of contact leggen met lotgenoten. Op deze manier kunnen PHRs patiënten ondersteunen bij het ontwikkelen van zelfmanagementvaardigheden en tegelijkertijd de afstand tot de zorgverlener verkleinen. Immers, patiënten kunnen vaak via een PHR op een laagdrempelige manier communiceren met hun zorgverleners. Op hun beurt kunnen de zorgverleners een beter beeld krijgen van de gezondheid van de patiënt vanwege de toegang tot gegevens die door de patiënt zijn verzameld. Op deze manier kunnen eventuele gezondheidsklachten sneller worden opgemerkt en dus worden behandeld.

Ondanks deze potentiele voordelen is er echter nog maar weinig bewijs voor de effectiviteit van PHRs. In de meeste evaluaties worden PHRs gezien als opzichzelfstaande technologieën die vooral voor de patiënt bedoeld zijn. Deze evaluaties bestaan vaak uit een voor- en een nameting (en vaak ook tussentijdse metingen op vaste tijdstippen) die met name geschikt zijn om inzicht te krijgen in of een toepassing effectief is geweest voor de patiënt. Echter, de implementatie van een patiëntenplatform is een complex proces, en dergelijke evaluaties geven geen verklaring voor waarom bepaalde effecten wel of niet werden gevonden, en hoe het proces van implementatie (waarbij ook de zorgverlener een belangrijke rol speelt) hieraan bij heeft gedragen. De toepassing blijft daarom een black box. Om te kunnen begrijpen wat de toegevoegde waarde is van PHRs, waarom ze deze toegevoegde waarde hebben, en waarom ze wellicht niet de verwachte impact hebben, moet deze black box 
worden geopend. Dit vraagt om een holistische benadering waarbij rekening wordt gehouden met de technologie, de gebruikers en de context waarin de toepassing wordt geïmplementeerd.

Dit proefschrift richt zich op de evaluatie van de implementatie van een PHR voor patiënten met diabetes mellitus type 2 , chronisch hartfalen, of COPD (e-Vita). Het eerste deel van dit proefschrift bestaat uit het conceptueel kader dat als basis dient voor de evaluatie van eVita. Het tweede deel omvat drie hoofdstukken die inzicht geven in de implementatie van eVita en de relatie tussen de gebruiker en de technologie en de invloed van de context hierop. Hierbij zullen we ons gaandeweg dit proefschrift richten op de volgende onderzoeksvragen:

1. Hoe wordt e-Vita op de lange termijn gebruikt?

2. Wie zijn de bedoelde en daadwerkelijke gebruikers van e-Vita?

3. Wordt e-Vita ervaren als gebruiksvriendelijk?

4. Zijn de stakeholders tevreden met de diensten die worden geleverd via de PHR?

5. Welke implementatiescenario's kunnen worden ontwikkeld voor de integratie van een PHR in de bestaande zorgprocessen om toegevoegde waarde te kunnen realiseren?

\section{Deel 1: Conceptueel kader}

In eHealth evaluaties is steeds meer aandacht voor hoe het daadwerkelijke gebruik van een technologie heeft bijgedragen aan de uitkomsten die werden gevonden. Dit wordt vaak gedaan door de adherentie aan de technologie te evalueren. Adherentie geeft aan in welke mate het gedrag van een persoon (in dit geval het gebruik van een technologie) in overeenstemming is met de overeengekomen aanbevelingen van bijvoorbeeld de zorgverlener (het beoogde gebruik). Er is echter tot op heden nog maar weinig bekend over wat het beoogde gebruik is van verschillende soorten eHealth technologie, en dus wat adherentie aan eHealth technologie is. In Hoofdstuk 2 wordt daarom een systematisch literatuuronderzoek (een systematic review) beschreven, waarbij we 62 studies hebben geëvalueerd waarin de adherentie aan eHealth technologie wordt gemeten. Dit hebben we gedaan door per studie de volgende drie elementen in kaart te brengen: 1) hoe is het gebruik gemeten; 2) of het beoogde gebruik is omschreven; en 3) of er op basis van theorie, eerder onderzoek, of rationale een onderbouwing is gegeven voor het beoogde gebruik.

De resultaten van het literatuuronderzoek lieten zien dat adherentie in meer dan de helft van de geïncludeerde studies wordt omschreven als 'hoe meer gebruik, hoe beter', zonder hierbij aan te geven wat het beoogde gebruik is. Wanneer het beoogde gebruik wel werd omschreven, was dit vaak niet gebaseerd op theorie, eerder onderzoek of rationale. Natuurlijk is het ook niet altijd mogelijk om het beoogde gebruik te definiëren (wanneer een technologie bijvoorbeeld heel nieuw is), maar in dat geval kan er ook niet van 'adherentie' 
worden gesproken. De conclusie van Hoofdstuk 2 is dan ook dat adherentie aan eHealth technologie een onderontwikkeld en vaak incorrect toegepast concept is in de bestaande literatuur.

Het gebruik van technologie wordt vaak geanalyseerd met behulp van logdata. Met behulp van logdata kan continu en objectief inzicht worden verkregen in hoe een technologie wordt gebruikt door de individuele gebruikers. Echter, tot op heden zijn de mogelijkheden van logdata nog niet ten volste benut. In Hoofdstuk 3 wordt daarom beschreven hoe logdataanalyses kunnen bijdragen aan een verbeterd inzicht in het gebruik van eHealth technologie. In dit hoofdstuk beschrijven we eerst wat logdata is en welke onderzoeksvragen beantwoord kunnen worden met logdata-analyses. Daarnaast beschrijven we welke eisen gesteld kunnen worden aan de kwaliteit van de logdata, geven we aanknopingspunten voor het verwerken van de ruwe logdata tot bestanden die geschikt zijn voor de data-analyse en beschrijven we enkele methodes voor de data-analyse. Ook beschrijven we wat de toegevoegde waarde is van logdata analyses voor zowel de wetenschap als de praktijk. Logdata analyses kunnen bijvoorbeeld nieuwe inzichten geven in hoe het gebruik van de technologie heeft bijgedragen aan de gevonden effecten van een technologie. Deze resultaten kunnen vervolgens inzicht geven in hoe de eHealth technologie en de onderliggende gedragsmodellen verbeterd kunnen worden. Logdata-analyses zijn daarom van grote toegevoegde waarde bij het openen van de black box van eHealth.

\section{Deel 2: De evaluatie van de implementatie van e-Vita}

Het tweede deel van dit proefschrift omvat drie hoofdstukken die inzicht geven in de implementatie van e-Vita. Om duurzame eHealth technologie te kunnen ontwikkelen, is het belangrijk inzicht te krijgen in de relatie tussen de technologie, de gebruiker en de context waarin de technologie wordt geïmplementeerd. Dit impliceert tegelijkertijd dat het functioneren van een technologie maar zelden in kaart kan worden gebracht wanneer het slechts vanuit één oogpunt of op één niveau wordt geëvalueerd. Daarom werd het functioneren van e-Vita op verschillende niveaus onderzocht.

Het doel van Hoofdstuk 4 is om meer inzicht te krijgen in hoe gebruikers van e-Vita Diabetes over het platform navigeren wanneer zij voor de eerste keer inloggen. Hiervoor hebben we de logdata gebruikt die is verzameld in de eerste zes weken na de lancering van een nieuwe versie van de PHR. Evaluaties lieten zien dat de meeste gebruikers de globale menustructuur van het systeem volgden, en daarbij meestal of één, of vijf verschillende onderdelen bezochten. Deze resultaten kunnen worden gebruikt voor het verbeteren van het ontwerp van het systeem. Op deze manier kan de aansluiting van het systeem bij de gebruikers worden verbeterd, waardoor de technologie ook op de langere termijn aantrekkelijk kan blijven voor de gebruikers. 
De Hoofdstukken 5 en 6 beschrijven de evaluatie waarmee het implementatieproces van eVita in kaart werd gebracht. Het doel van deze evaluaties is om meer inzicht te krijgen in hoe en waarom patiëntenplatformen van toegevoegde waarde kunnen zijn bij de zelfmanagementondersteuning van patiënten, maar tegelijkertijd ook zorgverleners kunnen ondersteunen bij het uitvoeren van hun dagelijkse werkzaamheden. Hiervoor is een zogenaamde 'convergent parallel mixed methods design' toegepast, waarbij kwalitatieve en kwantitatieve data naast elkaar werd verzameld, afzonderlijk werd geanalyseerd, en waarvan de resultaten vervolgens bij elkaar werden gebracht. Logdata werd gebruikt om inzicht te krijgen in hoe e-Vita op de lange termijn werd gebruikt. Daarnaast werden focusgroepen georganiseerd om inzicht te krijgen in het beoogde gebruik van e-Vita Diabetes volgens zorgverleners. Interviews met zorgverleners en usability tests met potentiele eindgebruikers werden gebruikt om de verschillen tussen het beoogde en het daadwerkelijke gebruik te kunnen verklaren.

Uit de interviews volgde dat de zorgverleners onvoldoende begeleiding hebben ervaren bij de integratie van e-Vita in de dagelijkse werkprocessen. Hierdoor bleef het onduidelijk hoe zij de verschillende onderdelen van het platform zodanig kunnen integreren in de dagelijkse werkroutines, dat deze ook daadwerkelijk van toegevoegde waarde zijn. De training die zorgverleners ontvingen was voornamelijk gericht op het verzamelen van data voor de evaluatiestudies, en daardoor waren zij onvoldoende voorbereid op wat er van hen werd verwacht bij het gebruik van e-Vita bij de zorg voor de patiënten. Hierdoor vonden zorgverleners het moeilijk om patiënten te motiveren om het platform ook daadwerkelijk te gaan gebruiken. e-Vita werd dan ook gezien als een extra belasting bovenop de reguliere taken, in plaats van als een ondersteuning.

Tijdens de focusgroepen gaven de zorgverleners aan dat de coaching via een PHR centraal zou moeten staan, en dat het gebruik van de overige onderdelen (zoals educatie en monitoring) hierbij aan zou moeten sluiten. Echter, uit de logdata bleek dat deze onderdelen niet werden gebruikt zoals beoogd. De usability tests lieten zien dat de gebruiksvriendelijkheid van het platform (met name de gebruikte terminologie) waarschijnlijk ook van invloed is geweest op het daadwerkelijke gebruik.

De conclusie van de evaluatie is dat het gebruik van het platform door patiënten sterk is beïnvloed door de attitude van de zorgverleners. Deze attitudes werden op hun beurt waarschijnlijk sterk beïnvloed door het gebrek aan ondersteuning die de zorgverleners hebben ervaren bij het daadwerkelijk inzetten van het platform in de dagelijkse werkzaamheden. Om dergelijke problemen te voorkomen, is het van groot belang om alle eindgebruikers al vroeg in de eHealth ontwikkeling te betrekken. Op deze manier kan de toegevoegde warde van de eHealth toepassing worden vergroot, en de daadwerkelijke implementatie worden gefaciliteerd. Korte en iteratieve ontwikkelcycli zijn cruciaal om 
problemen al in een vroeg stadium van de ontwikkeling te signaleren en om de kans op een succesvolle implementatie te vergroten. Bij het evalueren van de implementatie van eHealth technologie is een mixed methods benadering van grote toegevoegde waarde gebleken bij het vinden van verklaringen die niet gevonden hadden kunnen worden in experimenteel onderzoek.

De resultaten van het onderzoek hebben geleid tot een aantal geleerde lessen:

Les 1: Implementatie is geen post-design activiteit. e-Vita is ontwikkeld op basis van brainstormsessies met patiënten en zorgverleners om inzicht te krijgen in hun ervaringen met het leven met een chronische aandoeningen (patiënten) of het leveren van zorg aan chronisch zieken (zorgverleners). Op basis van deze informatie werd een eerste versie van het platform ontwikkeld. Echter, pas toen het platform al enige tijd in gebruik was genomen, bleek dat er tijdens de ontwikkeling onvoldoende aandacht is geweest voor de inbedding van het platform in het dagelijks leven en de werkprocessen van de eindgebruikers. Veel (potentiele) eindgebruikers gaven immers aan niet goed te weten hoe zij het platform dusdanig in kunnen zetten, dat het van toegevoegde waarde kan zijn. Helaas was het door de aard van de lopende studies niet meer mogelijk om het platform hierop aan te passen. Een van de belangrijkste conclusies van dit onderzoek is dan ook dat het van groot belang is om alle eindgebruikers en andere stakeholders te betrekken in alle fases van eHealth ontwikkeling. Korte en iteratieve ontwikkelcycli zijn cruciaal om eventuele problemen al vroeg op te sporen en de technologie aan te kunnen passen op basis van de aanbevelingen van de eindgebruikers en andere stakeholders. Hiermee start de implementatie van een toepassing al in de eerste fases van de ontwikkeling.

Les 2: Een platform implementeert niet vanzelf wanneer het wordt aangeboden aan de eindgebruikers. Uit eerdere (literatuur)onderzoeken weten we dat de aansluiting van technologie op de werkroutines en de interoperabiliteit met andere systemen belangrijke voorwaarden zijn voor de acceptatie van de technologie door de zorgverleners. Deze zorgverleners spelen op hun beurt een grote rol bij het motiveren van patiënten om een toepassing te gaan gebruiken. Training en ondersteuning van alle eindgebruikers in het gebruik van een technologie is dus onmisbaar. Aan de andere kant, als de toegevoegde waarde van een toepassing beperkt is, zal het nog steeds nauwelijks worden gebruikt. Het is dus van groot belang om toegevoegde waarde te creëren, nog voordat een technologie daadwerkelijk in gebruik wordt genomen. De eindgebruikers en andere stakeholders zijn daarom onmisbaar in alle fases van eHealth ontwikkeling, implementatie en evaluatie. 
Les 3: Een holistische benadering van evaluatie is cruciaal om inzicht te verkrijgen in het succes van een eHealth technologie. De resultaten van dit onderzoek laten zien dat het belangrijk is om te begrijpen hoe een technologie van toegevoegde waarde kan zijn voor bepaalde gebruikers in een bepaalde context. Deze conclusie sluit aan bij een recente aanbeveling van de Raad voor Volksgezondheid en Samenleving, die stelt dat naast de effectiviteit van een technologie ook de context en het implementatieproces belangrijke onderwerpen van onderzoek zijn. Deze aanbeveling impliceert korte en iteratieve evaluatiecycli die al vroeg in de ontwikkeling inzicht bieden in het potentiele succes of falen van een technologie. Vervolgens wordt een technologie alleen op een grotere schaal wordt ingezet als is gebleken dat de technologie aansluit bij de behoeftes van de eindgebruikers en de context. Logdata kan vervolgens continu inzicht geven in het daadwerkelijke gebruik van de technologie en kan aanknopingspunten bieden voor bijvoorbeeld interviews, focusgroepen en vragenlijsten. Op deze manier kan aanvullende informatie worden verkregen over de werkingsmechanismen van een technologie en warom het gebruik van een technologie (niet) tot de gewenste resultaten heeft geleid.

Ook leverde het onderzoek belangrijke aanbevelingen voor de toekomst op. Ten eerste is aanvullend onderzoek nodig om beter te begrijpen wat adherentie aan verschillende eHealth toepassingen is. Op deze manier kunnen we beter begrijpen hoe technologie gebruikers kan ondersteunen, bijvoorbeeld bij de ontwikkeling van zelfmanagementvaardigheden. Ook maakt dit de vergelijking van adherentie aan verschillende technologieën met hetzelfde doel eenvoudiger.

Ten tweede hebben we in Hoofdstuk 3 de potentie van logdata-onderzoek beschreven, bijvoorbeeld bij het voorspellen van adherentie en uitval, of om toepassingen te kunnen personaliseren op basis van het gebruik. Echter, de logdata moet hiervoor wel van voldoende kwaliteit zijn, en de (hoeveelheid) data die we in dit onderzoek konden verzamelen was van onvoldoende kwaliteit voor dergelijke analyses. Dit is voornamelijk het gevolg van de beperkte implementatie van het platform. Wanneer een toepassing beter is geïmplementeerd kan er meer gebruiksdata worden verzameld, waardoor we in de toekomst meer kunnen leren over hoe we technologie beter aan kunnen laten sluiten bij verschillende gebruikers in verschillende contexten.

Tot slot bieden de gegevens die via patiëntenplatformen worden verzameld (bijvoorbeeld gegevens over de gezondheid of leefstijl), mogelijkheden voor data-gestuurde coaching van chronisch zieke gebruikers. Echter, de verzamelde data kan niet zomaar worden gepresenteerd aan de gebruikers. Er is dus onderzoek nodig om meer inzicht te krijgen in hoe de data teruggegeven kan worden aan eindgebruikers, op zo'n manier dat de gebruiker deze informatie ook kan interpreteren. Hiervoor moet inzicht worden verkregen in de behoeftes 
van de gebruikers, en hoe data wordt gebruikt in de besluitvorming van patiënten en hun zorgverleners.

\section{Conclusie}

De belangrijkste conclusie van dit proefschrift is dat de technologie, de gebruiker en de context van het gebruik niet los van elkaar kunnen worden gezien bij de implementatie en evaluatie van patiëntenplatformen. Inzicht in de relatie tussen deze drie concepten was noodzakelijk om te kunnen begrijpen waarom de inzet van e-Vita niet heeft geleid tot de gewenste effecten.

Om toegevoegde waarde te creëren en de implementatie van eHealth toepassingen te faciliteren, is het van groot belang om alle eindgebruikers te betrekken in alle fases van eHealth ontwikkeling. Korte en iteratieve ontwikkelcycli zijn cruciaal om problemen al vroeg in de ontwikkeling op te sporen en op die manier de kans op een succesvolle implementatie te vergroten.

De gebruikte mixed methods benadering is van grote toegevoegde waarde gebleken bij de evaluatie van de implementatie van een platform voor patiënten met diabetes, chronisch hartfalen, of COPD. Op deze manier hebben we inzicht kunnen krijgen in het daadwerkelijke gebruik van het platform, en verklaringen kunnen vinden voor het beperkte gebruik. Deze resultaten waren niet naar boven gekomen wanneer de evaluatie zich alleen had gericht op de effectiviteit van een toepassing in een experimentele studie. Met dit onderzoek, waarbij we gebruik hebben gemaakt van geavanceerde onderzoeksmethodes, dragen we bij het aan het openen van de Black Box van eHealth.

182 | Samenvatting (Summary in Dutch) 


Toen ik begon met mijn promotie vond ik het maar onwerkelijk dat dit daadwerkelijk zou kunnen resulteren in een heus boekje met een eigen omslag, een ISBN-nummer en met mijn naam erop. En nu is het er gewoon! Het doen van een promotieonderzoek is iets wat je uiteindelijk zelf moet doen, maar tegelijkertijd niet kan zonder de hulp van anderen. Ik wil daarom graag de laatste bladzijdes van mijn proefschrift besteden aan het bedanken van de mensen die de afgelopen jaren hebben bijgedragen aan dit resultaat.

Lisette, mijn promotor. Onze eerste echte kennismaking was tijdens het sollicitatiegesprek. Ik zat toen, hartstikke zenuwachtig natuurlijk, in de gang te wachten terwijl jij druk heen en weer rende tussen de vergaderkamer en het toilet. Je was namelijk gebeten door de hond van de buren. Ik wist toen al dat, mocht ik aangenomen worden, het een bijzondere periode zou worden. En niets bleek minder waar. Er zijn meerdere dingen waarvoor ik je graag wil bedanken. Natuurlijk wil ik je graag bedanken voor alles wat ik van je heb geleerd. Maar boven alles wil ik je ook bedanken voor het vertrouwen dat je altijd in me hebt gehad, ook als het even niet liep zoals ik zou willen. Het was heel fijn dat je in zulke periodes altijd achter me stond. Verder, wil ik je bedanken voor alle kansen die ik van je heb gekregen om ook (kleine en wat grotere) projecten naast mijn promotie te doen, zoals de MOOC, Minddistrict, Twente TEACH, presentaties, workshops, congressen, et cetera. Ik heb vaak gedacht, waar heb ik nú weer 'ja' op gezegd (ook al was er niet eens altijd een vraag), maar ze hebben zeker mijn proefschrift gemaakt tot wat het nu is. Natuurlijk wil ik je ook graag bedanken voor alle gezelligheid en leuke momenten van de afgelopen jaren. We hebben samen heel wat plekken binnen en buiten Europa bezocht, waarbij je me haarfijn hebt uitgelegd hoe het nuttige met het aangename te verenigen.

Saskia, mijn co-promotor. Ik ben er trots op dat ik jouw eerste promovendus ben! Ik had me echt geen betere begeleider kunnen wensen. Dankjewel voor je nuchtere kijk op dingen, waarmee je mij zo nodig weer even met beide benen op de grond zette waardoor ik mijn blik helder(der) kon houden. Hiermee, en met je doorzettingsvermogen, ben je een groot voorbeeld voor mij.

Robbert, mijn andere promotor. We hebben elkaar voor het eerst ontmoet tijdens een sollicitatiegesprek voor een promotietraject in Groningen, waarvoor ik toen niet aangenomen ben. Toch kwamen we elkaar weer tegen in Enschede, waar je alsnog een van mijn promotoren werd. Zo gemakkelijk kwam je dus niet van me af! Ook al was je vooral op afstand betrokken, ik waardeer je mening en input altijd heel erg, net als het feit dat ik je altijd mocht benaderen voor allerlei onderzoeks(gerelateerde) -zaken. Het was fijn om iemand te hebben die de zaken (letterlijk) van een afstandje kon bekijken.

Prof. dr. Andrea Evers, prof. dr. Leonard Witkamp, dr. Rik Crutzen, prof. dr. ir. Hermie Hermens en dr. Stans Drossaert, ik vind het een grote eer dat $u$ in mijn promotiecommissie plaats wilde nemen. Prof. dr. Erwin Seydel, de voorzitter van mijn commissie. We kennen 
elkaar niet heel goed, maar ik kan mij nog goed herinneren dat toen ik tijdens het eerste uur van mijn eerste werkdag bij Annemarie zat, u even binnen kwam wandelen om haar gedag te zeggen. Ik vind het een eer dat $u$ de taak van voorzitter van de commissie wilt vervullen en op deze manier niet alleen bij het begin, maar ook bij de afsluiting van mijn promotie aanwezig bent.

Als je een kind vraagt wat hij of zij graag wil worden, zal het antwoord waarschijnlijk niet snel 'promovendus' of 'wetenschapper' zijn. Er is dus wel het een en ander voor nodig voordat je je op een promotieonderzoek durft te storten. Ik wil daarom een paar mensen bedanken die mij deze zetjes hebben gegeven. Stans, Roos, en Maureen, dank jullie wel dat jullie als mijn afstudeerbegeleiders mijn interesse voor eHealth met toegevoegde waarde voor de praktijk aangewakkerd hebben. Marieke Mulder en Paul van der Valk, hartelijk dank voor mogelijkheid die jullie mij hebben gegeven om na mijn afstuderen een kijkje te nemen in de onderzoekspraktijk. Paul en Patricia, dank jullie wel voor het vertrouwen dat jullie mij hebben gegeven voor die ene sollicitatie.

De (voormalige) stichting Zorg Binnen Bereik wil ik graag bedanken voor de mogelijkheid die ze me hebben geboden om dit onderzoek te kunnen doen.

Ook wil ik graag de projectpartners van Twente TEACH bedanken voor de samenwerking in het iMedisense project. Deze pilot was zo ontzettend leerzaam, en het heeft me geholpen om bepaalde resultaten van dit onderzoek in perspectief te plaatsen. Ik kijk er naar uit om iMedisense nog mooier te maken dan dat het al is.

Uiteraard wil ik ook mijn (oud-)collega's van de eHealth-club bedanken: Lisette, Annemarie, Aniek, Anne, Aranka, Hanneke, Jobke, Julia, Liseth, Magnus, Marit, Mark, Nadine, Nienke, Roberto, Robin, Saskia A. en Saskia K. Dank jullie wel voor jullie collegialiteit, gekkigheid, en (soms) georganiseerde chaos. Het is in ieder geval nooit saai! Het is zo fijn om mensen te hebben waar je altijd even binnen kunt lopen voor het nodige overleg. Ik ben trots op wat we doen en waar we voor staan, en dat ik daar deel van uit mag maken.

Daarnaast wil ik ook de collega's van de vakgroep PGT en de Rookies bedanken voor de collegialiteit en de samenwerking van de afgelopen jaren, de gezellige (vakgroep)uitjes en praatjes in het keukentje of bij het kopieerapparaat. Ik vind het een geruststellende gedachte dat ik met jullie (in bepaalde samenstellingen, dat wel ;-)...) een escape room kan trotseren!

De afgelopen jaren heb ik een hoop data kunnen verzamelen waarmee ik mijn boekje heb kunnen vullen. Dit had ik echter niet in mijn eentje gekund. Daarom wil ik graag Anita, DerkJan, Gerko, Kiki, Mara, Marloes, Stefan en Vivien bedanken. Zij zijn allemaal afgestudeerd op e-Vita-gerelateerde onderwerpen en ik wil hen daarom graag bedanken voor hun inzet bij het verzamelen en/of analyseren van stukjes van de data. Daarnaast waren er ook altijd leuke projecten die niet altijd direct gerelateerd waren aan dit promotieonderzoek, maar wel heel 
erg interessant zijn. Bij de afstudeerprojecten van Angelina, Anouk, Elisa, Christian, Deike, Isra, Jana, Kim, Mariska, Michelle, Noreen, Paul, Roberto, Saskia, Selina, Shannon, en Theresia was ik dan ook betrokken als (tweede begeleider). Ik wil jullie graag bedanken voor jullie inzet!

Marieke en Elize, dank jullie wel dat jullie deur (letterlijk) altijd open staat!

Speciale dank gaat uit naar mijn kamergenootjes Hanneke en Julia Keizer (there, I said it). Zonder jullie was het afgelopen jaar een stuk saaier geweest! Dank jullie wel voor de koffie, thee en flesjes water als ik het zelf vergat, de luiaard exposure-therapie, maar bovenal jullie gezelligheid en altijd een meedenkend en luisterend oor. Ik kijk er naar uit om voorlopig nog jullie kamergenoot te blijven!

Een jaar of zes geleden hadden we bij de master Gezondheidspsychologie het vak 'Capita Selecta', waarbij we een literatuuronderzoek moesten uitvoeren bij een specifieke onderzoeksvraag. Ik heb toen gekozen voor het onderwerp 'interventies ter bevordering van de handhygiëne op de Intensive Care', waarmee ik Jobke als begeleider kreeg toegewezen. Maar, er was nog iemand die haar onderzoek in dezelfde richting wilde doen, en dat was Nienke. Jobke bedacht daarom dat het net zo efficiënt zou zijn om enkele begeleidingsgesprekken samen te plannen. Wie had toen kunnen bedenken dat jullie nu, zes jaar later, allebei mijn paranimfen zijn... Ik vind het een heel fijn idee dat jullie bij de verdediging achter en naast mij staan! Dank jullie wel daarvoor.

Jobke, van mijn begeleider bij Capita Selecta werd je mijn kamergenoot. Ook al zijn we na een tijdje toch allebei naar een andere kamer verhuisd, we wisten elkaar (zowel binnen als buiten werktijd) toch altijd wel te vinden. Gelukkig ben je nu weer met enige regelmaat op de UT te vinden en heb ik de eer om samen met jou het Benefit project op te gaan pakken. Daar heb ik ontzettend veel zin in!

Nienke, mijn eeuwige steun en toeverlaat bij... tja, bij wat eigenlijk niet? Ik kan het zo gek niet verzinnen, of we hebben het er wel over gehad met elkaar. Het was voor mij dan ook niet meer dan logisch dat ik je als mijn paranimf hebt gevraagd.

Ik heb jullie (indirect) ook al op andere plekken genoemd, maar een vermelding in deze samenstelling mag absoluut niet ontbreken in dit dankwoord. Jobke, Laura, Nadine en Nienke, dank jullie wel voor alle koppen thee en koffie, hamburgers (mijn dankwoord kan niet bestaan zonder de hamburgers minstens één keer te noemen), dropjes, leuke uitjes, fijne gesprekken en steun van de afgelopen jaren. Jullie zijn me erg dierbaar geworden, en ik vind het ook gewoon echt niet leuk als we elkaar mislopen vanwege bijvoorbeeld drukke agenda's, vakanties, zwangerschapsverloven, andere banen, en zelfs emigraties. Het is gewoon allemaal nét wat leuker als jullie er wel zijn. 
Karin, van studiegenoten in Groningen werden we collega's in Hengelo, en inmiddels zijn we ook al weer wat jaartjes vriendinnen. Dank je wel voor je gezelligheid en goede gesprekken onder het genot van een biertje of een mok thee en de nodige eetafspraken. Ook die dingen waren erg belangrijk in de afgelopen jaren!

Nelleke, al vanaf de middelbare school lopen onze levens min of meer parallel aan elkaar. Zo zijn we tegelijk naar Groningen gegaan, waar we samen heel wat gezelligheid hebben beleefd. Daarna zijn we in dezelfde periode weer naar Twente verhuisd; jij met een omweg vanuit Voorburg, ik vanuit Groningen. Nu wonen we alweer een tijdje op verschillende plekken, maar gaan we drie weken na elkaar allebei promoveren! We zien elkaar helaas niet heel vaak, maar het contact is gebleven en het is altijd fijn om even met je bij te kunnen praten. Dankjewel daarvoor.

Lieve oma, dank je wel voor alle wijze levenslessen die je me hebt meegegeven, zoals 'de oogn los, of de portemonnee los' en 'woar't kump hangt ook geen schilderiej' $n$ ' (wat betreft die laatste, ik zie nog zo voor me hoe je aardbeienjam over een banaan 'kwakte', terwijl we er volgens het recept een mooi bootje van moesten maken. Dat vond ik zó erg!). Maar boven alles hoor ik je heel vaak in mijn hoofd zeggen, 'jij traag, een ander graag', en heb je me geleerd dat je je altijd zelf moet kunnen redden zonder van anderen afhankelijk te zijn. Dit zijn twee lessen die ontzettend waardevol bleken te zijn, ook (of juist) bij een promotie. Ook al vind ik de verdediging heel spannend, ik kijk er ontzettend naar uit om jou hierbij op de eerste rij te zien zitten.

Luuk, mijn broertje. Ik betrap mijzelf er vaak op dat ik je nog steeds zo noem, ook al zijn de rollen inmiddels steeds vaker omgedraaid en heb je mij de afgelopen jaren van allerlei adviezen voorzien. Bijvoorbeeld, welke laptop of wandsteun voor de tv moet ik kopen? En wat zijn leuke plekken op Bali (om vervolgens naar Bari op vakantie te gaan)? En de meest spannende: Hoe herken je vals geld, en wat zijn de do's en don'ts bij het verkopen van een laptop? Eigenlijk moet ik je dus ook gewoon 'mijn broer' gaan noemen. Het feit dat ik dat niet altijd doe, doet niks af van het feit dat ik hartstikke trots op je ben!

Om nog even terug te komen op wat ik hiervoor al schreef: ik weet niet beter dan dat ik 'iets' in het ziekenhuis wil worden, 'net als mama'. Alleen, in het ziekenhuis (en überhaupt in de zorg) werken heel veel mensen in heel veel verschillende functies. Probeer dan maar eens in één keer de goede keuze te maken... Uiteindelijk is dit allemaal goedgekomen hoor, en mag ik nu al een paar jaar onderzoek doen naar een van de meest fascinerende ontwikkelingen in de gezondheidszorg. En hoe leuk is het dat ik daarmee zo af en toe ook bij mijn moeder aan tafel zit? 
Lieve pap en mam, wat mag ik mij gelukkig prijzen dat jullie mij altijd de ruimte hebben gegeven om uit te vinden wat ik wilde (ook als dat weer een verhuizing betekende, of toch nog een (pre-) master). Jullie hebben mij altijd gestimuleerd om het beste uit mijzelf te halen, maar dan wel op zo'n manier dat ik nog steeds gewoon met een onvoldoende thuis durfde te komen. Het is het cliché der clichés, maar zo waar: zonder jullie had dit boekje er niet gelegen en ik kan jullie niet genoeg bedanken voor alles wat jullie voor mij doen, en hebben gedaan.

Lieve Roderik, wat een geluk dat je in mijn leven bent gekomen. De afgelopen twee jaren waren roerig en zijn er heel wat kilometers verreden. Er wordt wel eens gezegd dat het goed is om elkaar te missen. Nu was dat in het begin ook zeker het geval, maar toch vind ik het nog veel fijner om elke dag samen thuis te komen (en dat is echt niet alleen omdat de laatste tijd het eten klaar stond als ik weer eens laat thuis was). De 'wereld van de wetenschap' was voor jou een hele nieuwe, maar toch probeer je altijd te begrijpen wat ik vertel en met de vragen die je stelt, sla je vaak de spijker op z'n kop. Dankjewel voor alles wat je me hiermee hebt geleerd en hebt doen inzien. Dikke kus. 


Publications \& Other Output 


\section{Journal articles}

Sieverink, F., Kelders, S., \& Van Gemert-Pijnen, J. (forthcoming). When does usage become adherence? A systematic review to clarify the concept of adherence to eHealth technology.. Journal of Medical Internet Research. doi:10.2196/jmir.8578

Sieverink, F., Kelders, S., Poel, M., \& Van Gemert-Pijnen, J. (2017). Opening the Black Box of Electronic Health: Collecting, Analyzing, and Interpreting Log Data. JMIR Research Protocols, 6(8): e156.

Sieverink, F., Kelders, S., Braakman-Jansen, L., \& Van Gemert-Pijnen, J. (2014). The added value of log file analyses of the use of a personal health record for patients with type 2 diabetes mellitus: preliminary results. Journal of Diabetes Science and Technology, 8(2), 247255.

Sieverink, F., Braakman-Jansen, L., Roelofsen, Y., Hendriks S., Sanderman, R., Bilo, H. \& Van Gemert-Pijnen, J. (2014). The diffusion of a personal health record for patients with type 2 diabetes mellitus in primary care. International Journal on Advances in Life Sciences, 6(3\&4), 177-183.

\section{Co-authored}

Wagenaar, K., Rutten, F., Klompstra, L., Bhana, Y., Sieverink, F., Ruschitzka, F., Seferovic, P. M., Lainscak, M., Piepoli, M. F., Broekhuizen, B., Strömberg, A., Jaarsma, T., Hoes, A., \& Dickstein, K. (2017). 'heartfailurematters.org', an educational website for patients and carers from the Heart Failure Association of the European Society of Cardiology: objectives, use and future directions. European Journal of Heart Failure. doi:10.1002/ejhf.917

Siemons, L., Sieverink, F., Braakman-Jansen, A., van Gemert-Pijnen, J., Vollenbroek, W., \& Van de Wijngaert, L. (2016). Big Data for personalized healthcare. International journal on advances in systems and measurements, 9(3/4), 220-229.

Van Vugt, M., de Wit, M., Sieverink, F., Roelofsen, Y., Hendriks, S., Bilo, H., \& Snoek, F. (2016). Uptake and effects of the e-Vita personal health record with self-management support and coaching, for type 2 diabetes patients treated in primary care. Journal of Diabetes Research. doi:10.1155/2016/5027356

Roelofsen, Y., Hendriks, S., Sieverink, F., van Vugt, M., van Hateren, K., Snoek, F., De Wit, M., Gans, R., Groenier, K., Van Gemert-Pijnen, J., Kleefstra, N., \& Bilo, H. (2014). Design of the eVita diabetes mellitus study: effects and use of an interactive online care platform in patients with type 2 diabetes (e-VitaDM-1/ZODIAC-40). BMC Endocrine Disorders, 14(1), 22. 
Roelofsen, Y., Hendriks, S., Sieverink, F., Landman, G., Groenier, K., Bilo, H., \& Kleefstra, N. (2014). Differences between patients with type 2 diabetes mellitus interested and uninterested in the use of a patient platform (e-VitaDM-2/ZODIAC-41). Journal of Diabetes Science and Technology, 8(2), 230-237.

\section{Book Chapters}

Sieverink, F. (2014). Slimmer eHealth ontwikkelen en implementeren met de CeHRes Roadmap. In C. Politiek, \& R. Hoogendijk (Red.), Co-Creatie eHealth Boek. eHealth, technisch kunstje of pure veranderkunde? (pp. 242-250).

\section{Conference contributions}

\section{Presentations and workshops}

Sieverink, F., Poel, M., Kelders, S., \& Van Gemert-Pijnen, J. (2017). Log data analyses to personalize eHealth technology: predictors for adherence. Presented at the sixth annual conference of the Association for Researchers in Psychology and Health (ARPH), Leiden, the Netherlands, February 2, 2017.

Sieverink, F., Kelders, S., Poel, M., \& Van Gemert-Pijnen, J. (2016). How to collect, analyze and interpret log data from eHealth technology. Presented at Supporting Health by Technology VII, Groningen, the Netherlands, May 27, 2016.

Van Gemert-Pijnen, J., Sieverink, F., Siemons, L., \& Braakman-Jansen, A. (2016). Big Data for Personalized and Persuasive Coaching via Self-monitoring Technology., Workshop at the Eighth International Conference on eHealth, Telemedicine, and Social Medicine (eTELEMED 2016), Venice, Italy, April 24-28, 2016.

Sieverink, F., Kelders, S., Akkersdijk, S., Poel, M., Siemons, L., \& Van Gemert-Pijnen, J. (2016). Work in progress: a protocol for the collection, analysis, and interpretation of log data from eHealth technology. In O. Kulyk, L. Siemons, H. Oinas-Kukkonen, \& J. Van Gemert-Pijnen (Red.), Proceedings of the Fourth International Workshop on Behavior Change Support Systems. Co-located with the 11th International Conference on Persuasive Technology (pp. 56-60), Salzburg, Austria, April 5-7, 2016.

Sieverink, F., Siemons, L., Braakman-Jansen, A., \& Van Gemert-Pijnen, J. (2015). What does Big Data mean for Personalized Medicine? Research in progress. Presented at Medicine $X$, Stanford, United States, September 23-27, 2015. 
Sieverink, F., Akkersdijk, S., Kelders, S., Braakman-Jansen, A., Brandenburg, B., \& Van GemertPijnen, J. (2014). Personal Health Records for Self-Management: Critical Factors for Implementation in Primary Care. Presented at Medicine X, Stanford, United States, September 4-7, 2014.

Van Gemert-Pijnen, J., Kulyk, O., Wentzel, J., Sieverink, F., Beerlage-de Jong, N., \& Kelders, S. (2014). How to improve eHealth interventions in Health Psychology and Behavioral Medicine? Workshop at the 13th International Congress of Behavioral Medicine (ICBM2014), Groningen, the Netherlands, August 20-23, 2014.

Sieverink, F., Kelders, S., Braakman-Jansen, A., \& van Gemert-Pijnen, J. (2014). The Uptake and Impact of a Personal Health Record for Patients with Type 2 Diabetes Mellitus in Primary Care: a research protocol for a backward and forward evaluation. Paper presented at the Sixth International Conference on eHealth, Telemedicine, and Social Medicine (eTELEMED 2014). Barcelona, Spain, March 23-27, 2014.

\section{Posters}

Beerlage-de Jong, N., Wrede, C., Van Gemert-Pijnen, J., \& Sieverink, F. (2017). Storyboarding persuasion to match personality traits. Poster presented at the 12th International Conference, PERSUASIVE 2017, Amsterdam, The Netherlands, April 4-6, 2017.

Sieverink, F., Siemons, L., Braakman-Jansen, A., \& Van Gemert-Pijnen, J. (2016). Internet of Things \& Personalized Healthcare. Poster presented at the Special Topic Conference of the European Federation for Medical Informatics. Paris, France, April 17-19, 2016.

Sieverink, F., Poel, M., Kelders, S., Braakman-Jansen, A., \& Van Gemert-Pijnen, J. (2015). Log Data Analysis via Machine Learning to Make eHealth More Persuasive. Poster presented at the $10^{\text {th }}$ International Conference, PERSUASIVE 2015, Chicago, United States, June 4-5, 2015.

Akkersdijk, S., Sieverink, F., Kelders, S., Braakman-Jansen, A., \& Van Gemert-Pijnen, J. (2014). Strong Points for the Implementation of Personal Health Records in Primary Care. Poster presented at the 13th International Congress of Behavioral Medicine (ICBM2014), Groningen, the Netherlands, August 20-23, 2014.

\section{Other output}

Hofdijk, J., Séroussi, B., Lovis, C., Ehrler, F., Sieverink, F., Ugon, A., \& Hercigonja-Szekeres, M. (Red.). (2016). Proceedings of the EFMI Special Topic Conference 2016. Amsterdam, The Netherlands: IOS Press.

Kelders, S., Kip, H., Sieverink, F., \& van Gemert-Pijnen, J. (2016). eHealth: Combining Psychology, Health and Technology (MOOC). FutureLearn (www.futurelearn.com/courses/ehealth). 
Floor Sieverink is a researcher at the Centre for eHealth and Wellbeing Research of the University of Twente. Her PhD research focused on the evaluation of a personal health record (PHR) to understand what differences PHRs can make in health care, why PHRs make these differences, and why PHRs may or may not have the expected impact.

She combined quantitative data (log data) and qualitative data (interviews, focus groups, usability tests) in a mixed methods approach to understand how patients use a PHR in the context of their care, and how PHRs can add value to the working routines of caregivers. This holistic mixed methods approach of eHealth evaluation is rather unique.

Her research interests include the implementation and evaluation of eHealth, and persuasive design to create adherence.

ISBN: 978-90-365-4417-7

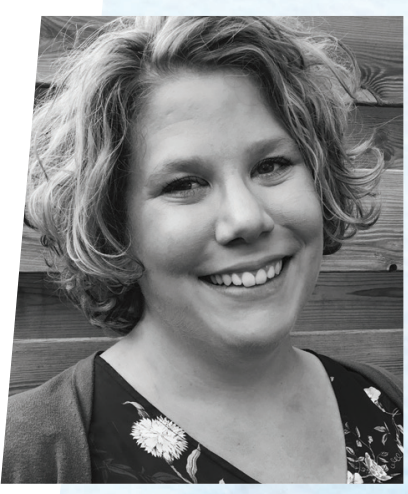

